\title{
Abundance Derivations for the Secondary Stars in Cataclysmic Variables from Near-Infrared Spectroscopy
}

\author{
Thomas E. Harrison ${ }^{1,2}$ \\ Department of Astronomy, New Mexico State University, Box 30001, MSC 4500, Las \\ Cruces, NM 88003-8001 \\ tharriso@nmsu.edu
}

Received — 


\begin{abstract}
We derive metallicities for 41 cataclysmic variables (CVs) from near-infrared spectroscopy. We use synthetic spectra that cover the $0.8 \mu \mathrm{m} \leq \lambda \leq 2.5 \mu \mathrm{m}$ bandpass to ascertain the value of $[\mathrm{Fe} / \mathrm{H}]$ for $\mathrm{CVs}$ with K-type donors, while also deriving abundances for other elements. Using calibrations for determining $[\mathrm{Fe} / \mathrm{H}]$ from the $K$-band spectra of M-dwarfs, we derive more precise values for $\mathrm{T}_{\mathrm{eff}}$ for the secondaries in the shortest period CVs, and examine whether they have carbon deficits. In general, the donor stars in CVs have sub-solar metallicities. We confirm carbon deficits for a large number of systems. CVs with orbital periods $>5 \mathrm{hr}$ are most likely to have unusual abundances. We identify four CVs with CO emission. We use phase-resolved spectra to ascertain the mass and radius of the donor in U Gem. The secondary star in U Gem appears to have a lower apparent gravity than a main sequence star of its spectral type. Applying this result to other $\mathrm{CVs}$, we find that the later-than-expected spectral types observed for many CV donors is mostly an affect of inclination. All of the magnetic CVs, except the low accretion rate polar MQ Dra, have donors with subsolar metallicities. We find that two systems with unusual spectra, EI Psc and QZ Ser, that have large excesses of sodium, and extreme deficits of carbon. Synthetic spectra that have a reduced abundance of hydrogen are best able to explain the spectra of these two objects.
\end{abstract}

Key words: infrared: stars — stars: cataclysmic variables — stars: abundances stars: individual (RX And, AE Aqr, VY Aqr, UU Aql, TT Ari, SS Aur, SY Cnc, V436 Cen, WW Cet, WX Cet, TT Crt, EM Cyg, EY Cyg, SS Cyg, EX Dra, MQ Dra, YY/DO Dra, U Gem, AH Her, AM Her, EX Hya, VW Hyi, RZ Leo, ST LMi, CW Mon, V426 Oph, V2051 Oph, CN Ori, CZ Ori, V1309 Ori, IP Peg, LS Peg, RU Peg, GK Per, KT Per, EI Psc, VV 
Pup, V893 Sco, MR Ser, QZ Ser, AR UMa, CH UMa, TW Vir)

${ }^{1}$ Visiting Observer, W. M. Keck Observatory, which is operated as a scientific partnership among the California Institute of Technology, the University of California, and the National Aeronautics and Space Administration.

${ }^{2}$ Visiting Astronomer at the Infrared Telescope Facility, which is operated by the University of Hawaii under contract from NASA. 


\section{Introduction}

Cataclysmic variables $(\mathrm{CVs})$ result when the cool, low mass secondary in a binary contacts its Roche lobe and transfers material to the white dwarf primary. To arrive at this stage, the standard evolutionary paradigm involves having the more massive component in the binary evolve off the main sequence and engulf its companion in a short-lived common envelope (CE) phase (e.g., Iben \& Livio 1993). During the CE phase, the outer atmosphere of the primary star is removed by interaction with the secondary star, and this interaction leads to either a stellar merger, or a more compact binary (Politano \& Weiler 2007). Angular momentum loss, such as through "magnetic braking" (Verbunt \& Zwaan 1981), eventually leads to a semi-contact binary and a CV is born. In most of the early population synthesis calculations (cf., Howell et al. 2001, and references therein) the vast majority of donor stars in CVs were expected to be unevolved. If true, the donor stars should generally appear to be similar to their main sequence counterparts, except for the effects introduced by the mass loss process. Thus, while they will have a slightly different mass-radius relationship due to the secondary being out of thermal equilibrium, their photospheres should not show any abundance peculiarities.

The standard evolutionary paradigm though is slowly changing to reflect the fact that many long period CVs appear to have donors with unusual masses and/or radii. This suggests that the secondaries in some subset of CVs have undergone significant evolution prior to becoming interacting binaries. Podsiadlowski et al. (2003) constructed a population synthesis for CVs that allowed for evolved secondaries with initial masses of up to $1.4 \mathrm{M}_{\odot}$. Inclusion of these objects allowed them to explain the wide dispersion in the observed temperatures of donor stars in CVs with $\mathrm{P}_{\text {orb }}>5 \mathrm{hr}$. This result confirmed the conclusions of Baraffe \& Kolb (2000), that long period CVs are dominated by systems containing evolved secondaries. Recently, Goliasch \& Nelson (2015) have produced a new population 
synthesis calculation that includes detailed nuclear evolution. They confirm the results of Podsiadlowski et al., showing that there is a large range of masses and radii for donors in CVs with $\mathrm{P}_{\text {orb }}>5 \mathrm{hr}$ when nuclear evolved secondary stars are considered. Two other interesting results from this study are that CVs with evolved secondaries do not have a period gan! and they can evolve to much shorter periods than predicted by models where evolved donors are not considered. Podsiadlowski et al. suggest that the secondaries that have undergone pre-CV evolution should show evidence for nuclear processing, in particular, products produced via the $\mathrm{CNO}$ cycle.

Two alternative paths that might allow the donor stars in CVs to acquire peculiar abundance patterns are for them to accrete such material during the original common envelope phase, or during the briefer common envelope phase that results from a classical nova eruption. Marks \& Sarna (1998) have investigated a large number of scenarios for the composition of the surface layers of CV donor stars that have accreted both types of material, and find that significant changes are possible. A common theme amongst these models are large deficits of carbon. In a series of papers we have presented near-IR $K$-band spectra that demonstrated that the secondaries of many CVs appeared to have significant carbon deficits (Harrison et al. 2004a, 2005ab, 2009). Recently, Harrison \& Hamilton (2015, "H\&H") used synthetic spectra to quantify that SS Cyg, RU Peg, and GK Per had carbon abundances that were 10 to $30 \%$ of the solar value. In contrast, however, they found that the metallicities $([\mathrm{Fe} / \mathrm{H}])$ of the donor stars were solar, or only slightly subsolar.

The study by H\&H was limited to the $K$-band and, as they describe, there are not many strong atomic absorption lines in that bandpass to derive more precise values of $[\mathrm{Fe} / \mathrm{H}]$, nor enough spectral lines from any species other than carbon to allow derivation of their abundances. To remedy this issue, we have developed the capability to generate

\footnotetext{
${ }^{1}$ The observed dearth of CVs with $2 \mathrm{hr} \leq \mathrm{P}_{\text {orb }} \leq 3 \mathrm{hr}$ (see Kolb et al. 1998).
} 
synthetic spectra that cover the $I J H K$ bandpasses. This allows us to investigate the abundances of other important elements for the subset of CVs we have observed using cross-dispersed spectrographs. From modeling these data we find a wide range of values for $[\mathrm{Fe} / \mathrm{H}]$, and for $[\mathrm{C} / \mathrm{Fe}]$. In addition, however, we find a number of $\mathrm{CVs}$ that appear to have unusual abundances of aluminum, magnesium, and/or sodium. Due to the lack of an extensive line list (and other uncertainties discussed below) our abundance analysis has been limited to CVs whose secondaries have spectral types earlier than M0. Recently, relationships have been developed to extract metallicities for $\mathrm{M}$ dwarfs. Using those relationships it is possible to examine the cooler secondary stars found in short period CVs for abundance anomalies.

We describe the observations in the next section, the generation of synthetic spectra in Section 3, the results on CVs with K-dwarf secondaries in Section 4, the process and data needed to generate metallicities for $\mathrm{M}$ dwarfs in Section 5, discuss our results in Section 6, and present our conclusions in Section 7.

\section{Observations}

The majority of the new data presented below was obtained with SPEX on the IRTF. SPEX was used in cross-dispersed mode generating spectra spanning the wavelength range from 0.8 to $2.45 \mu \mathrm{m}$. In the $K$-band, the dispersion is $5.4 \AA /$ pix. Nearby A0 $\mathrm{V}$ stars were used for telluric correction, and these data were telluric-corrected and flux- and wavelength-calibrated using the SPEXTOOL package (Cushing et al. 2004). SPEXTOOL attempts to fit, and remove the $\mathrm{H}$ I lines in the telluric A star. While this process can work quite well, many times there remain residuals at the positions of the strongest $\mathrm{H} I$ lines. Thus, the line strengths and profiles of strong H I absorption or emission features in these data should not be taken too seriously. A log of all of our observations for previously 
unpublished data is presented in Table 1, and it lists the UT date, start and stop times, the length of the exposure times used for each object, the instrument used, the orbital period, the orbital phase at the mid-point of the observational sequence (except for U Gem where full orbits were covered), and the outburst state for each of the CVs.

We also present previously unpublished spectra obtained with NIRSPEC2 ${ }^{2}$ on Keck. We used NIRSPEC in low-resolution, single order mode with a 0.38 " slit. The grating tilt was set so as to cover the wavelength region $2.04 \mu \mathrm{m} \leq \lambda \leq 2.46 \mu \mathrm{m}$, with a dispersion of $4.27 \AA /$ pixel. We employed the two-nod script, and used 4 minute exposure times for all of the program CVs. To correct for telluric absorption, we observed bright A0V stars located close to the program objects so as to minimize their relative differences in air mass. These data were reduced using the IDL routine REDSPEQ3 specially developed for NIRSPEC.

Finally, we used TripleSped (Wilson et al. 2004) on the Apache Point Observatory (APO) $3.5 \mathrm{~m}$ telescope to obtain phase-resolved near-IR spectra of U Gem over two orbital periods, and for $50 \%$ orbital period coverage of QZ Ser. TripleSpec is similar to SPEX in that it provides cross-dispersed spectra covering the near-IR, from $0.95 \mu \mathrm{m}$ to $2.46 \mu \mathrm{m}$, at a resolution of $\mathrm{R} \sim 3500$. The 1.1 " slit was used, and all exposures were four minutes in length. These data were reduced using "Triplespectool," a version of SPEXTOOL modified by Michael Cushing for use at APO. Nearby A0V stars were used to provide telluric correction.

In the following we will also be analyzing the spectra of CVs observed in just the $K$-band with the IRTF using SPEX, at Keck with NIRSPEC, or at the VLT with ISAAC.

\footnotetext{
2 http://www2.keck.hawaii.edu/inst/nirspec/nirspec.html

${ }^{3}$ https://www2.keck.hawaii.edu/inst/nirspec-old/redspec/index.html

${ }^{4}$ http://www.apo.nmsu.edu/arc35m/Instruments/TRIPLESPEC/
} 
All of these data have been previously published in Harrison et al. (2004a, 2005ab, 2007a, 2009), and Hamilton et al. (2011). Unless otherwise noted, all of the spectra have been Doppler corrected using published radial velocity data and orbital ephemerides.

\section{Synthetic Spectra for the $I J H K$ Bands}

In $\mathrm{H} \& \mathrm{H}$ we described the construction of a Python-based program (named "kmoog") that used MOOG (Sneden 1973) to generate a large grid of synthetic $K$-band spectra, and to autonomously search through this grid to derive the best fitting result for each program object. As described there, the focus of that effort was in modeling the CO features, and the capability to investigate the abundances of other elements was not implemented. With the desire to expand our coverage to shorter wavelengths, we decided to abandon further development of kmoog in favor of using "SPECTRUM" by R. Gray ${ }^{5}$. The main driver for this decision was the existence of a line list covering the visual and near-IR ${ }^{6}$. As discussed in $\mathrm{H} \& \mathrm{H}$, the process of constructing and validating a $K$-band line list was quite onerous, and one we wished to avoid if possible.

As in $\mathrm{H} \& \mathrm{H}$, we fixed the micro-turbulence to $2 \mathrm{~km} \mathrm{~s}^{-1}$ for all synthetic spectra, a value that appears to best represent normal stars (Husser et al. 2013, Smith et al. 2013). Likewise, all of our synthetic spectra were run with $\log g=4.5$. Cesetti et al. (2013) have examined the use of moderate resolution data from the IRTF Spectral Library to derive diagnostics for cool stars. They found very few features that allow for accurate quantification of $\log g$ for higher gravity stars. Given the large rotation velocities of CV donors, the limited spectral resolution, and the presence of numerous emission features, it

\footnotetext{
${ }^{5}$ http://www.appstate.edu/ grayro/spectrum/spectrum.html

${ }^{6}$ http://www.appstate.edu/grayro/spectrum/specftp.html
} 
is not possible to constrain the surface gravity from the data discussed herein.

Unlike $k m o o g$, SPECTRUM is not natively designed to use a multiple processor machine that greatly aids in speeding-up the process of generating large grids of models. To allow us to quickly construct large grids of synthetic spectra using SPECTRUM, we used the GNU shell tool "parallel' with our 32 core Linux workstation. We found that we could generate models as quickly with SPECTRUM + parallel, as with $\mathrm{kmoog}$.

The other main difference between the use of SPECTRUM vs. $k m o o g$ is that the latter uses MARCS7 atmosphere models when computing synthetic spectra, while SPECTRUM was designed for the input of Kuruct $2^{8}$ models. Thus, before we began our program we first had to insure that the results generated with SPECTRUM matched those generated by $k m o o g$ (see $\mathrm{H} \& \mathrm{H}$ for validation of $k m o o g$ ). We ran a small grid of models with identical parameters using both $k m o o g$ and SPECTRUM. In addition, we also translated the MARCS models used for this test into the format used by Kurucz to determine if the input atmosphere model resulted in significant differences. The spectra produced by the three processes were identical as far as we could tell.

As in $\mathrm{H} \& \mathrm{H}$, the next step was to validate the line list using template $\mathrm{K}$ dwarf spectra. For this process we used the same set of dwarfs as in $\mathrm{H} \& \mathrm{H}$ (the data are from the IRTF Spectral Library, Cushing et al. 2005). We found that in general, the "luke.nir" (covering $6800 \AA \leq \lambda \leq 1 \mu \mathrm{m}$ ) and "luke.ir" (covering $1 \mu \mathrm{m} \leq \lambda \leq 4 \mu \mathrm{m}$ ) line lists were quite reliable. We did adjust the oscillator strengths for some of the strongest Mg I lines, but otherwise few changes were necessary.

In Fig. 1 we present the spectra in the $I J H K$ bandpasses for the $\mathrm{K}$ dwarf templates

\footnotetext{
${ }^{7}$ http://marcs.astro.uu.se/

${ }^{8}$ http://kurucz.harvard.edu/grids.html
} 
with the best fitting models calculated using SPECTRUM. We identify the strongest atomic and molecular absorption features in each panel to show the redundancy of spectral features for each of the elements, and as a guide for when we discuss features in the spectra of the program CVs. Except for the CN bandhead near $1.1 \mu \mathrm{m}$, the models reproduce all of the strongest spectral features. The spectral regions near $1.1 \mu \mathrm{m}$ and $2.0 \mu \mathrm{m}$ are afflicted with strong telluric features that compromise the observations.

As we did for the $K$-band in $\mathrm{H} \& \mathrm{H}$, to determine the best fitting models we constructed heat maps from a $\chi^{2}$ analysis. Those for the $J$-band, covering the spectral types from K0V to $\mathrm{K} 5 \mathrm{~V}$, are shown in Fig. 2. We tabulate the best fitting value for $\mathrm{T}_{\text {eff }}$ and $[\mathrm{Fe} / \mathrm{H}]$ for each of the template stars from a $\chi^{2}$ analysis of the synthetic spectral grid for each of the bandpasses in Table 2. In this table we also list the averages from those fits, and their nominal values (see Table 2 in $\mathrm{H} \& \mathrm{H}$ ). In $\mathrm{H} \& \mathrm{H}$, the typical error bars in the determination of $[\mathrm{Fe} / \mathrm{H}]$ and $\mathrm{T}_{\text {eff }}$ from the SPEX data using just the $K$-band were \pm 0.25 and $\pm 250 \mathrm{~K}$, respectively. As shown in Table 2, the errors in both quantities, when averaging over the four bandpasses, are all smaller, and easily within the combined error bars of our models and the averages of the published values. We must note that the temperature grid of the Kurucz atmospheres steps in intervals $250 \mathrm{~K}$, while the abundance grid at each temperature was $[\mathrm{Fe} / \mathrm{H}]=+0.5,+0.25,0.0,-0.1,-0.2,-0.3,-0.5$, and -1.00 (we also interpolated between the -0.5 and -1.00 models to create atmospheres with $[\mathrm{Fe} / \mathrm{H}]=-0.75)$. Thus, there is an uneven quantization in the model values of $[\mathrm{Fe} / \mathrm{H}]$.

The average of the differences in temperature between our models and the nominal values is only $+22 \mathrm{~K}$, suggesting our temperature derivations using these low resolution spectra are reliable: $\sigma= \pm 110 \mathrm{~K}$. For the values of $[\mathrm{Fe} / \mathrm{H}]$, however, the average of the differences is -0.11 , suggesting that we tend to derive slightly lower metallicities than the means of the published values. Thus, while the formal error on our derivation of $[\mathrm{Fe} / \mathrm{H}]$ 
for the templates is only $\sigma= \pm 0.04$, the offset we find, and the actual error bars on the nominal values, suggests that the true standard deviation on our metallicities is at least a factor of two larger than this.

In the following subsection will use this same technique for all of the CVs with K-type secondaries. $\chi^{2}$ minimization will be used to derive $\mathrm{T}_{\mathrm{eff}},[\mathrm{Fe} / \mathrm{H}]$, and $[\mathrm{C} / \mathrm{Fe}]$. Note that the $\chi^{2}$ minimization process was only run on select regions of the spectra in each of the photometric bands. As described in $\mathrm{H} \& \mathrm{H}$, we avoid regions with strong emission features intrinsic to the $\mathrm{CV}$, and regions where strong telluric features compromise the calibration. In the $K$-band, the analysis was first conducted on the spectral region from $2.19 \mu \mathrm{m}$ to $2.29 \mu \mathrm{m}$ to arrive at values for $\mathrm{T}_{\text {eff }}$ and $[\mathrm{Fe} / \mathrm{H}]$. Then the region from 2.19 to $2.36 \mu \mathrm{m}$ was used to find the best fitting value of $[\mathrm{C} / \mathrm{Fe}]$. If we were to include the $\mathrm{CO}$ region into the initial $\chi^{2}$ analysis for the $K$-band, the results are greatly biased by the strength of the CO features (which might have a non-solar abundance). In the $J$-band the analysis region was constrained to $1.16 \leq \lambda \leq 1.26 \mu \mathrm{m}$.

In the $I$ - and $H$-bands, the available spectral regions for analysis are compromised by $\mathrm{H} \mathrm{I}$ and/or Ca II emission lines. There were no CVs for which a $\chi^{2}$ analysis was possible in the $I$-band. While there are gaps between the Paschen series in the $I$-band, no strong absorption features are visible in those gaps. For most of the CVs, the $\chi^{2}$ minimization calculation for the $H$-band was run over the wavelength interval $1.55 \leq \lambda \leq 1.67 \mu \mathrm{m}$. There were CVs, however, where the Brackett series made this impossible (e.g., GK Per). In all cases, we visually inspected the reasonableness of the fit of the final model to the data in the $I$ - and $H$-bands that was derived from the $J$ - and $K$-band analysis.

For several of the CVs it is clear that the abundances of magnesium, sodium, and/or aluminum appeared anomalous. In those cases we ran models with the best-fit values for $\mathrm{T}_{\text {eff }}$ and $[\mathrm{Fe} / \mathrm{H}]$, but with small changes in the abundances of the element that seemed 
peculiar. We then derived the value for the deficit/enhancement of that element from visual inspection. The final results for all of the objects are listed in Table 3. In this table, and in the remainder of the paper, CV subclasses, orbital periods and inclinations are from

the online version of the Ritter \& Kolb (2003) catalog ("RKCat"), unless otherwise noted. In Table 3 we list the object name, the CV subtype, $\mathrm{P}_{\text {orb }}, \mathrm{T}_{\text {eff }}$ of the donor derived from the modeling, its metallicity $([\mathrm{Fe} / \mathrm{H}])$, its carbon abundance $([\mathrm{C} / \mathrm{Fe}])$, listing any other abundance anomalies in the final column. In several cases, we set the abundance to a prescribed value before modeling, when this occurs, the value in the table is followed by an "=" sign.

\subsection{The Derived Abundances for CVs with K-type Secondaries Using Cross-Dispersed SPEX Data}

Using the methods described above, we now present the results from modeling the program objects. We want to stress again that the presence of $\mathrm{H}$ I emission throughout the range of these spectra severely limits the $\chi^{2}$ analysis. Thus, the precision of the derived metallicities for the CVs are almost certainly poorer than those derived from the K-type templates. The following subsections on the individual CVs are ordered by orbital period. As shown in Knigge et al. (2011), CVs with orbital periods above 5 hrs are expected to have masses of $\mathrm{M}_{2} \sim 0.6 \mathrm{M}_{\odot}$ for their donor stars. The spectral type of a main sequence star with such a mass is M0V. Thus, we will start with the assumption that all CVs with $\mathrm{P}_{\text {orb }}>5 \mathrm{hr}$ will probably have $\mathrm{K}$ type donors (though our analysis may lead to a different answer). 


\subsubsection{GK Persei}

GK Per is a long period $\left(\mathrm{P}_{\text {orb }}=48.1 \mathrm{hr}\right) \mathrm{CV}$ that had a classical nova eruption in 1901 (see Harrison et al. 2013). The secondary star in this system is clearly a subgiant. H\&H modeled the $K$-band spectrum of GK Per observed with both Keck NIRSPEC and IRTF SPEX, finding $\mathrm{T}_{\text {eff }}=5100 \mathrm{~K},[\mathrm{Fe} / \mathrm{H}]=-0.125$, and $[\mathrm{C} / \mathrm{Fe}]=-1.0$. The cross-dispersed SPEX data are shown in Fig. 3. As detailed in Table 3, we find $\mathrm{T}_{\text {eff }}=5000 \mathrm{~K},[\mathrm{Fe} / \mathrm{H}]$ $=-0.3$, and $[\mathrm{C} / \mathrm{Fe}]=-0.5$. As discussed in $\mathrm{H} \& \mathrm{H}$, the lower $\mathrm{S} / \mathrm{N}$ of the $\mathrm{GK}$ Per data set meant more uncertain results than for their other two CVs. With the wider spectral coverage, we are better able to constrain the metallicity and carbon abundance, leading to slightly revised results (but within the error bars of both results). In addition to a strong deficit of carbon, we also find that all of the $\mathrm{Mg}$ I lines (see Fig. 1 for identifications) are weaker than they should be for this spectral type. We conclude that the magnesium abundance in the subgiant donor is $50 \%$ of the solar value.

\subsubsection{EY Cygni}

EY Cyg is a low inclination dwarf nova of long orbital period, $\mathrm{P}_{\text {orb }}=11.02 \mathrm{hr}$ (Echevarria et al. 2007). Echevarria et al. found a spectral type for the secondary of K0V, in agreement with that derived by Kraft (1962). Gänsicke et al. (2003) found an anomalous $\mathrm{N} \mathrm{V} / \mathrm{C}$ IV emission line ratio that suggested a deficit of carbon. Analysis of FUSE and HST spectroscopy by Sion et al. (2004) found a carbon abundance that was $20 \%$ solar, and a silicon abundance that was $10 \%$ of solar. From the SPEX data (Fig. 4) we derive $\mathrm{T}_{\text {eff }}=$ $5250 \mathrm{~K}$, consistent with a spectral type of $\mathrm{K} 0 \mathrm{~V}$, and $[\mathrm{Fe} / \mathrm{H}]=0.0$. We confirm the reported carbon deficit, finding $[\mathrm{C} / \mathrm{Fe}]=-0.5$. While the Si I lines in the $J$-band do appear to be weaker than expected, the $\mathrm{S} / \mathrm{N}$ of these data are too poor to determine an absolute silicon abundance. 


\subsubsection{AE Aquarii}

AE Aqr is an intermediate polar (IP), a system with a highly magnetic white dwarf that is spinning much more rapidly than the orbital period: $\mathrm{P}_{\text {orb }}=9.88 \mathrm{hr}, \mathrm{P}_{\text {spin }}=0.55$ min. Harrison et al. (2007a) have already published the $I J H K$ spectra from SPEX, and noted the near-absence of the CO features in the $K$-band. Those data, and the best fitting model, are plotted in Fig. 5. We derive a temperature of $\mathrm{T}_{\text {eff }}=4750 \mathrm{~K}(\sim \mathrm{K} 4),[\mathrm{Fe} / \mathrm{H}]=$ 0 , and $[\mathrm{C} / \mathrm{Fe}]=-1.0$. The derived temperature is in agreement with the phase-dependent spectral type range of $\mathrm{K} 0(\phi=0.4,0.9)$ to $\mathrm{K} 4(\phi=0.65)$, found by Echevarria et al. (2008). Some of this spectral type range might be due to starspots, as Doppler tomography found that $\sim 18 \%$ of the northern hemisphere of the secondary star is covered with spots (Watson et al. 2006). Except for the deficit of carbon, no other spectroscopic anomalies are evident.

\subsubsection{RU Pegasi}

RU Peg, $\mathrm{P}_{\text {orb }}=8.99 \mathrm{hr}$, was modeled by $\mathrm{H} \& \mathrm{H}$, and they found $\mathrm{T}_{\text {eff }}=4700 \mathrm{~K},[\mathrm{Fe} / \mathrm{H}]$ $=0$, and $[\mathrm{C} / \mathrm{Fe}]=-0.75$. The SPEX data are presented in Fig. 6. The best fitting model has $\mathrm{T}_{\text {eff }}=5000 \mathrm{~K},[\mathrm{Fe} / \mathrm{H}]=-0.3$, and $[\mathrm{C} / \mathrm{Fe}]=-0.4$. While the global metallicity in our new result is substantially lower, the absolute carbon deficit has not changed. Obviously, the values of $[\mathrm{Fe} / \mathrm{H}]$ and $[\mathrm{C} / \mathrm{Fe}]$ are intertwined, and both models suggest a large, total deficit of carbon. It is not as clear why we now find a hotter star with a lower metallicity (though again, within the combined error bars of both results). As discussed in H\&H (see their Fig. 18), with a limited data set, hotter models with super-solar abundances are nearly equivalent with cooler models with sub-solar abundances. Here we have the opposite trend. The increased wavelength coverage makes the new determination more reliable.

In the following we will use the newly constrained properties of the secondary stars to 
estimate the distances to CVs using spectroscopic parallaxes. When possible, we compare these spectroscopic distances to those derived from astrometric parallaxes. Harrison et al. (2004b) used the Fine Guidance Sensors (FGS) on the HST to measured a parallax for RU

Peg that leads to a distance of $282 \pm 20$ pc. The 2MASS data for RU Peg, obtained during quiescence, is: $(J-H)=0.44,(H-K)=0.16$, and $K=10.46$. These colors are consistent with the K4 temperature we derive from the spectra. The parallax, however, gives $\mathrm{M}_{\mathrm{K}}=$ 3.2 , whereas a main sequence $\mathrm{K} 4 \mathrm{~V}$ has $\mathrm{M}_{\mathrm{K}}=4.48$. The secondary star would have to be a G4V to explain the observed $\mathrm{M}_{\mathrm{K}}$ ! The donor in RU Peg is three times more luminous than a main sequence star of the same spectral type.

\subsubsection{SS Cygni}

$\mathrm{H} \& \mathrm{H}$ modeled the $K$-band data for SS Cyg and found $\mathrm{T}_{\text {eff }}=4700 \mathrm{~K},[\mathrm{Fe} / \mathrm{H}]=-0.25$, and $[\mathrm{C} / \mathrm{Fe}]=-0.50$. They noted that the $\mathrm{Mg}$ I line at $2.28 \mu \mathrm{m}$ was weaker than expected, but could not derive an abundance for magnesium. Modeling the $I J H K$ spectra, Fig. 7 , we find very similar results: $\mathrm{T}_{\text {eff }}=4750 \mathrm{~K},[\mathrm{Fe} / \mathrm{H}]=-0.3$, and $[\mathrm{C} / \mathrm{Fe}]=-0.40$. With the more expansive wavelength coverage, we conclusively find that there is a deficit of magnesium, $[\mathrm{Mg} / \mathrm{Fe}]=-0.3$.

We can use the temperature we found above, and published values that characterize the secondary star, to generate an updated spectroscopic parallax. Bitner et al. (2007) conducted a radial velocity study of SS Cyg and found that the secondary star was 10 to $50 \%$ larger than a main sequence dwarf, which implies an absolute magnitude that is brighter by 0.21 to 0.88 mag. In their paper they assumed a $\mathrm{K} 4.5 \mathrm{~V}$ star. Here, the temperature is closer to a $\mathrm{K} 3.5 \mathrm{~V}$. For such an object $\mathrm{M}_{\mathrm{K}} \simeq 4.40$, with their offsets in absolute magnitude, the limits become $3.52 \leq \mathrm{M}_{\mathrm{K}_{\mathrm{SS}}} \leq 4.19$. With $\langle K\rangle=9.45$ (Harrison et al. 2007b), the spectroscopic distance to SS Cyg is $102 \leq d \leq 153$ pc. Unfortunately, 
this range includes all of the published astrometric distances for this object. The mean, $128 \mathrm{pc}$, is very similar to the distance that resulted from the revised FGS analysis? 9 Harrison \& McArthur (2016). As will be discussed below, evidence suggests that the spectral types derived from our spectroscopic observations are inclination angle-dependent. For an object with the binary inclination angle of SS Cyg, $i \sim 45^{\circ}$ (Harrison et al. 2007b), the true spectral type of the secondary is likely to be one full subclass earlier, $\sim \mathrm{K} 2.5 \mathrm{~V}$. Therefore, with $\mathrm{M}_{\mathrm{K}} \simeq 4.2$, the resulting distance estimate, assuming an unbloated main sequence donor, is $112 \mathrm{pc}$; in agreement with the radio parallax of Miller-Jones et al. (2013). Given the result for RU Peg, combined with the fact that the secondary star in SS Cyg has a subsolar metallicity, and deficits of both carbon and magnesium, suggests that the assumption of a main sequence luminosity for the donor is suspect.

\subsubsection{RX Andromedae}

RX And has an orbital period of $5.03 \mathrm{hr}$, and Schreiber et al. (2002) suggest it has characteristics of both Z Cam dwarf novae (DNe), and VY Scl stars. The Z Cam subclass of DNe have "standstills," where the system brightens to a level well above minimum, but stays a magnitude or more below maximum. VY Scl stars, on the other hand, frequently show very low states, where the mass transfer drops to low levels, and the system fades by a magnitude or more below its typical minimum light level. VY Scl stars do not show dwarf novae outbursts. RX And clearly has dwarf novae outbursts, but also extended standstills and very low states. The shorter orbital period of RX And suggests that its donor star may have a spectral type of M0, or later. We will discuss deriving the metallicities for $\mathrm{M}$

\footnotetext{
${ }^{9} \pi=7.41 \pm 0.20$ mas. Note that this value for the parallax differs from that quoted in the abstract of that paper due to further analysis of the data set.
} 
stars in $\S 4$. The line lists used for modeling the spectra of K dwarfs with SPECTRUM are not expected to be reliable for $\mathrm{M}$ dwarfs. However, our main reluctance in modeling $\mathrm{M}$ dwarf data using SPECTRUM (or MOOG) is the lack of the incorporation of water vapor absorption.

At M0V, the water vapor absorption features are not very strong, and our continuum division process can easily remove them. Thus, we ran a grid of models that included $\mathrm{T}_{\text {eff }}=3750 \mathrm{~K}$ to attempt to constrain the nature of the secondary star in RX And. To demonstrate how well the line list works for this lower temperature, in Fig. 7 we also plot our model spectra on top that of the IRTF spectral template HD 19305. There are no published values for the metallicity of HD 19305, however, using the techniques described below $(\S 4)$, we derived $[\mathrm{Fe} / \mathrm{H}]=0.0$, and used this value in generating model spectra. The resulting synthetic spectrum does a reasonable job of matching HD 19305 in the $I$, $J$, and $K$-bands, though nearly all of the lines from Ca I are poorly fit by the model spectrum in these bandpasses. The model $H$-band spectrum does a very poor job at reproducing the observations. This is presumably due to ignoring molecules such as $\mathrm{H}_{2} \mathrm{O}$.

We found that the best fit for RX And was achieved with the $\mathrm{T}_{\text {eff }}=3750 \mathrm{~K}$ model, and $[\mathrm{Fe} / \mathrm{H}]=-0.3$, but the donor star has several abundance anomalies. While the carbon deficit was mild, $[\mathrm{C} / \mathrm{Fe} /]=-0.1$, the abundances of both $\mathrm{Mg}$ and $\mathrm{Al}$ were significantly diminished (e.g., Mg I at 1.173 and $1.771 \mu \mathrm{m}$; the Al I doublets 1.314 and $2.11 \mu \mathrm{m}$ ). The donor star in RX And could have a normal carbon abundance if the effective temperature was slightly higher. In agreement with the results of Sepinsky et al. (2002) who found a solar abundance for carbon. Sepinsky et al. also found a normal abundance for silicon, and the Si I lines in the $J$-band for RX And appear to be at their proper strength. The Mg I and Al I lines were reproduced for HD 19305 by the synthetic spectrum. The strengths of both of these species decline with decreasing temperature, but this would require a much 
cooler donor star than M0 to explain their strength here.

Using the techniques described in $\S 4$, the metallicity analysis procedure suggests that the donor in RX And has a spectral type of $\mathrm{M} 2$, and $[\mathrm{Fe} / \mathrm{H}]=+0.07$. This is a later spectral type than would be expected at this orbital period. As shown in Table 1, the secondary was close to superior conjunction, thus any irradiation would have made it appear hotter than it would on average. Obviously, synthetic spectra using models with cooler effective temperatures are necessary to quantify the properties of the donor in RX And. A cooler star would have weaker Mg I and Al I lines, so their deficiencies can thus be explained.

It is also important to note the strong Ca II triplet emission in the $I$-band. These three lines are narrower than the nearby H I Paschen series, suggesting they have an origin on the secondary star. We will see further evidence of Ca II emission below. Such lines could be used to generate a radial velocity curve for the donor objects in systems where the absorption features cannot be easily seen.

\subsection{Abundance Analysis for CVs with Expected K Dwarf Donors Using $K$-band Data Only}

Over the years, we have collected $K$-band spectra for a large number of CVs. Most of these data were presented in Harrison et al. (2004a, 2005ab). The spectral types and carbon abundances were qualitatively examined in those papers. Here we have generated models

for those objects. The results for these objects are listed in Table 3 , we briefly discuss each object below. As noted earlier, having only a single bandpass of moderate resolution data leads to poorer constraints on both $\mathrm{T}_{\text {eff }}$ and $[\mathrm{Fe} / \mathrm{H}]$. However, the results for some objects indicate very low values for the metallicities, and argue for deeper, multi-bandpass infrared spectroscopy. Some of the data analyzed below have low $\mathrm{S} / \mathrm{N}$. Where the $\chi^{2}$ analysis is 
ambiguous, we have chosen the least unusual model as the final result.

\subsubsection{SY Cancri}

SY Cnc is a long period dwarf nova of the Z Cam subclass. The $K$-band spectrum was presented in Harrison et al. (2004a), where a spectral type of G2 was derived. Connon Smith et al. (2005) obtained a radial velocity curve for this object and find a low mass for the secondary star $\left(\mathrm{M}_{2}=0.36 \mathrm{M}_{\odot}\right)$, and conclude that its spectral type is near M0. While the $K$-band data are of low quality, the strength of the blue Ca I features, Na I, Ca $\mathrm{I}$, and $\mathrm{CO}$ are most consistent with $\mathrm{T}_{\text {eff }}=5500 \mathrm{~K},[\mathrm{Fe} / \mathrm{H}]=0.0$, and $[\mathrm{C} / \mathrm{Fe}]=0.0$. The temperature we derive corresponds to a spectral type of G8V, and is more consistent with the type of donor expected at this orbital period (Knigge 2006). Note that SY Cnc was in outburst at the time of this spectrum, and irradiation might result in finding a hotter temperature for the secondary star. The visibility of the donor in $K$-band spectroscopy during outburst suggests that an excellent radial velocity curve and $v \sin i$ rotation rate could be obtained for this bright object $(K=10.9)$ using NIR spectroscopy. Such data are needed to better determine the masses of both components in this system.

\subsection{2. $C H$ Ursae Majoris}

Harrison et al. (2004a) presented the $K$-band spectrum of $\mathrm{CH}$ UMa and derived a spectral type of K7 \pm 2 , consistent with the M0 type found by Friend et al. (1990). This is cooler than expected for this orbital period $\left(\mathrm{P}_{\text {orb }}=8.232 \mathrm{hr}\right)$. UV spectroscopy reveals that $\mathrm{CH}$ UMa has a large $\mathrm{N} \mathrm{V/C} \mathrm{IV} \mathrm{ratio,} \mathrm{suggesting} \mathrm{a} \mathrm{deficit} \mathrm{of} \mathrm{carbon} \mathrm{(Gänsicke} \mathrm{et}$ al. 2003). Sion et al. (2007) found that their best fit white dwarf model for the FUSE

spectrum of CH UMa indicated very low abundances for both carbon and silicon. The $\chi^{2}$ 
analysis indicates that the best fit temperature is near $\mathrm{T}_{\text {eff }}=4750 \mathrm{~K}$. Such a value would be closer to that expected for this orbital period. While the donor star appears to have a near-solar metallicity, the CO features in the $K$-band spectrum are exceptionally weak, and our analysis finds $[\mathrm{C} / \mathrm{Fe}]=-1.0$. The Si I lines in the $K$-band spectrum are not prominent, even at solar metallicities for these temperatures, but they do not appear to be unusual in the spectrum of $\mathrm{CH}$ UMa.

\subsubsection{V1309 Orionis}

In Harrison et al. (2005a) we presented the $K$-band spectra of five polars with M-type companions, and found that they all appeared to have normal carbon abundances (see below). Since that time, we obtained a $K$-band spectrum of the very long period polar V1309 Ori $\left(\mathrm{P}_{\text {orb }}=7.98 \mathrm{hr}\right.$ ) using NIRSPEC on Keck (Howell et al. 2010). We have generated a grid of synthetic model spectra and find that, assuming a solar metallicity, the temperature is between $4750 \mathrm{~K} \leq \mathrm{T}_{\text {eff }} \leq 5000 \mathrm{~K}$. Hotter temperatures do not have the strong $\mathrm{NaI}$ and $\mathrm{Ca}$ I features seen here, while cooler temperatures would require reduced metallicities to reproduce the strength of these lines. Our result for $\mathrm{T}_{\text {eff }}$ is similar to the late K-type derived by Howell et al. (2010), but is in contrast to Garnavich et al. (1994) who suggested an MOV.

Given the poor quality and limited spectral range of the data for V1309 Ori, we cannot fully exclude cooler donor stars. CO is clearly not in emission in this object, nor are their any signs of cyclotron emission (see Harrison \& Campbell 2015). We conclude that carbon is highly deficient no matter the spectral type. For $\mathrm{T}_{\text {eff }}=4750 \mathrm{~K}$, we derive $[\mathrm{C} / \mathrm{Fe}]=-1.0$. Mg I at $2.28 \mu \mathrm{m}$ is also very weak. The profile of the $\mathrm{Mg}$ I line at $2.1 \mu \mathrm{m}$ is corrupted by He I emission. To reproduce the strength of the $2.28 \mu \mathrm{m}$ line implies that $[\mathrm{Mg} / \mathrm{Fe}] \sim$ -0.50. Szkody \& Silber (1996) found that the N V/C IV ratio was large, suggesting a 
carbon deficit. Thus, there is at least one polar whose secondary star does not appear to be "normal." It is interesting that this deviation occurs in the longest period polar.

\subsubsection{EM Cygni}

EM Cyg is an eclipsing Z Cam dwarf novae that has an orbital period of $6.982 \mathrm{hr}$. Thus the donor star should have a mid-K spectral type. Harrison et al. (2004a) had a difficult time classifying its $K$-band spectrum, finding that some features suggested a K0V, while others were more consistent with a K5V. This ambiguity can be understood as the best fitting family of models from our analysis have both sub-solar metallicities, and modest deficits of carbon. Godon et al. (2009) have modeled FUSE data for EM Cyg and found that nitrogen, sulphur and silicon are highly overabundant, while carbon appeared to have a solar abundance. Unfortunately, our spectrum of EM Cyg is too noisy to examine the strength of the Si I lines, though absorption features are present at the locations of each of the strongest such lines.

\subsubsection{V426 Ophiuchi}

V426 Oph is a bright, Z Cam-type dwarf nova that was observed by Harrison et al. (2004a). They found an early K spectral type, consistent with the K3 type derived by Hessman (1988). Evidence is mounting that V426 Oph is actually an IP (Ramsay et al. 2008 , and references therein). The favored synthetic model spectrum has $\mathrm{T}_{\text {eff }}=4750 \mathrm{~K}$, $[\mathrm{Fe} / \mathrm{H}]=0.0$, and with $[\mathrm{C} / \mathrm{Fe}]=-0.5$, producing an excellent fit to the SPEX data. 


\subsubsection{TT Crateris}

$K$-band spectra for TT Crt have been presented in Harrison et al. (2004a) where a spectral type of K5V was found. Szkody et al. (1992) and Thorstensen et al. (2004a) found that the donor star in TT Crt $\left(\mathrm{P}_{\text {orb }}=6.44 \mathrm{hr}\right)$ is easily seen in visual spectroscopy, and appears to be a mid to late K-type star. Our best fitting model agrees with these three estimates, having $\mathrm{T}=4500 \mathrm{~K}$, and $[\mathrm{Fe} / \mathrm{H}]=0.0$. The $\mathrm{CO}$ features are weaker than they should be at this spectral type, and we derive a carbon abundance of $[\mathrm{C} / \mathrm{Fe}]=-0.50$.

\subsubsection{AH Herculis}

AH Her is a DNe with a period of $6.19 \mathrm{hr}$, and would be expected to have a mid/late-K type secondary. The $K$-band data for AH Her was obtained with SPEX and, as described in Harrison et al. (2004a), this DNe was going into outburst at the time of these observations. The data quality is poor, but the two best fitting families of models have either $\mathrm{T}_{\text {eff }}=$ $5500 \mathrm{~K},[\mathrm{Fe} / \mathrm{H}]=0$, and $[\mathrm{C} / \mathrm{Fe}]=0$, or $\mathrm{T}_{\text {eff }}=4750 \mathrm{~K},[\mathrm{Fe} / \mathrm{H}]=-0.75$, and $[\mathrm{C} / \mathrm{Fe}]=$

-0.4. The hotter model matches most of the spectrum except for the Ca I lines near 1.98 $\mu \mathrm{m}$. Given the orbital period, the lower temperature for the donor would be expected, and indicates a low metallicity secondary with a significant carbon deficit. Our result may have been compromised by irradiation and/or dilution, though the system was near inferior conjunction.

\subsubsection{CZ Orionis}

CZ Ori is a DNe with a period of $5.25 \mathrm{hr}$. Ringwald et al. (1994) estimated that the secondary star has a spectral type of M2.5 \pm 1 from the presence of TiO absorption. While the $K$-band spectrum of CZ Ori is poor (see Fig. 9), it is clear that the CO features have 
an unusual profile, suggesting $\mathrm{CO}$ emission is present. Using the technique described in $\mathrm{H} \& \mathrm{H}$, we added $\mathrm{CO}$ emission spectra to the models generated by SPECTRUM and find that a donor star with $\mathrm{T}=4250 \mathrm{~K},[\mathrm{Fe} / \mathrm{H}]=-1.0$ and $[\mathrm{C} / \mathrm{Fe}]=0.0$, combined with a $\mathrm{CO}$ emission spectrum broadened to a velocity of $800 \mathrm{~km} \mathrm{~s}^{-1}$, reproduces the observations. This is only the second CV shown to have CO emission features, the other being the ultra-short period system WZ Sge (Howell et al. 2004, Harrison 2016); several additional examples will be discussed below. A temperature of $4250 \mathrm{~K}$ corresponds to a spectral type near K7. Hotter stars fit nearly equally as well, and have larger metallicities (e.g., $[\mathrm{Fe} / \mathrm{H}]=-0.75$ for $\left.\mathrm{T}_{\mathrm{eff}}=4750 \mathrm{~K}\right)$. Cooler stars require much lower metallicities to fit these data, and also need to have carbon deficits. If the metallicity was truly as low as indicated here, the $\mathrm{TiO}$ features would probably have been too weak to have extracted a spectral type from optical data. Higher quality, multi-bandpass infrared spectroscopy of this object is needed.

\subsubsection{EX Draconis}

EX Dra has $\mathrm{P}_{\text {orb }}=5.04 \mathrm{hr}$, and Baptista et al. (2000) found $\mathrm{M}_{2}=0.54 \mathrm{M}_{\odot}$. This mass for the secondary would suggest a mid-M type star, while the expected spectral type for this period would be near the K/M boundary. Harrison et al. (2004a) derived a spectral type of K7 from the SPEX data. They noted that the CO features and the Mg I lines were weaker than expected at this spectral type. Our analysis finds a best fitting model of $\mathrm{T}_{\text {eff }}=$ $4000 \mathrm{~K},[\mathrm{Fe} / \mathrm{H}]=-0.5,[\mathrm{C} / \mathrm{Fe}]=-0.2$, and $[\mathrm{Mg} / \mathrm{Fe}]=0.0$. Except for the carbon deficit, there are no obvious abundance anomalies. 


\section{Deriving Metallicities for CVs with M-type Donor Stars}

In our infrared spectroscopic surveys of shorter period CVs, we presented evidence that the carbon abundances in many of the secondaries with M spectral types appeared deficient. The main issue with such analysis was that the template stars to which we were comparing the CV spectra had known spectral types, but unknown metallicities. Thus, if the spectral type of the CV donor was somewhat uncertain, comparing it to the spectra of several template stars might lead to erroneous conclusions due to the possibility that the template stars might have significantly non-solar values for $[\mathrm{Fe} / \mathrm{H}]$. A proper qualitative analysis requires us to be able to compare stars of known properties to those that we seek to characterize.

While models for the structure of the atmospheres of $M$ dwarfs have improved (e.g., Baraffe et al. 2015), it is still difficult to generate synthetic spectra that are adequate for abundance measures due to incomplete line lists, and the presence of water vapor features throughout the NIR (however, see Önehag et al. 2012, and Bean et al. 2006). Thus, alternative methods have been sought that calibrate various spectral features for M dwarfs with known metallicities determined using their association with hotter stars, for which such derivations are simpler (see Rojas-Ayala et al. 2010, Mann et al. 2013, 2014, and Newton et al. 2014). Mann et al. (2013, 2014) have performed a rigorous analysis and developed relationships to quantify the value of $[\mathrm{Fe} / \mathrm{H}]$ for dwarfs spanning the spectral type range K5 to M9. These relationships use the equivalent widths of the Na I doublet at $2.20 \mu \mathrm{m}$, and the Ca I triplet at $2.26 \mu \mathrm{m}$, along with an "H2O index" (from Covey et al. 2010) to derive $[\mathrm{Fe} / \mathrm{H}]$ from $K$-band spectra. The $\mathrm{H} 2 \mathrm{O}$ index gives the temperature dependence, and thus an estimate of the spectral type.

In Mann et al. (2013) the relationship for determining $[\mathrm{Fe} / \mathrm{H}]$ using these three features for earlier spectral types (<M4) is given, while in Mann et al. (2014), a different 
formulation is found that covers the range M4 to M9. We can now derive $[\mathrm{Fe} / \mathrm{H}]$ for the various template $\mathrm{M}$ dwarfs that can used for comparison to the CV spectra, as well as attempt to derive $[\mathrm{Fe} / \mathrm{H}]$ directly for the donor stars. Note that due to the weakness of the $\mathrm{CO}$ features in some of the $\mathrm{CVs}$, the value of the $\mathrm{H} 2 \mathrm{O}$ index could be erroneous (since its reddest continuum bandpass is at $2.37 \mu \mathrm{m})$. In these cases, we can use the calibration by Newton et al. for $[\mathrm{Fe} / \mathrm{H}]$ that only uses the $\mathrm{Na}$ I doublet. That relationship is only valid for spectral types from M2 to M6. The $1 \sigma$ error bars on $[\mathrm{Fe} / \mathrm{H}]$ for the Newton et al. (2014) relation is \pm 0.12 dex, while Mann et al. $(2013,2014)$ claim an error of \pm 0.07 dex for their calibration.

There is one vitally important caveat that could dramatically alter our metallicity estimates using these techniques, and that is the dependence of the derived value of $[\mathrm{Fe} / \mathrm{H}]$ on the abundances of carbon and oxygen. Veyette et al. (2016) have used PHOENIX models of cool stars to attempt to probe how the derived values of $[\mathrm{Fe} / \mathrm{H}]$ using the above techniques are dependent on the $\mathrm{C} / \mathrm{O}$ abundance ratio. Less carbon in the atmosphere means more $\mathrm{H}_{2} \mathrm{O}$, leading to changes in the continuum of the cooler $\mathrm{M}$ dwarfs. As an example, for an object with $[\mathrm{C} / \mathrm{Fe}]=-0.2$ and $[\mathrm{O} / \mathrm{Fe}]=0.0$, the derived abundance for a solar metallicity star, using the Mann et al. (2013) relations with $\mathrm{T}_{\text {eff }}=3500 \mathrm{~K}$, would be $[\mathrm{Fe} / \mathrm{H}]=-0.15$. For $\mathrm{T}_{\text {eff }}=3000 \mathrm{~K}$, it would be $[\mathrm{Fe} / \mathrm{H}]=-0.4$. Thus, if the carbon abundance is low, we could dramatically underestimate the true metallicity of the secondary star. We will return to this subject below.

With the ability to constrain $[\mathrm{Fe} / \mathrm{H}]$ for the templates and donor stars, we can fully investigate whether the lines of any other elements are at unusual strengths in the $\mathrm{CV}$ secondaries. Like the preceding section, we first begin with the analysis of cross-dispersed spectra for the individual program objects ordered by orbital period. We then examine the objects with only $K$-band data. Unless otherwise indicated, we have used visual inspection 
to determine the best fitting spectral type and to note any abundance anomalies. The results are listed in Table 3. The value of $\mathrm{T}_{\text {eff }}$ listed in this table uses the calibration in Rajpurohit et al. (2013) to turn estimated spectra types into temperatures. That calibration assumes solar metallicity.

\subsection{Cross-Dispersed Spectroscopy}

\subsubsection{SS Aurigae}

SS Aur is a typical DNe with an orbital period of $4.39 \mathrm{hr}$, and Harrison et al. (2005b) found a spectral type of M4 for the donor star. Using the Mann et al. relationship gives $[\mathrm{Fe} / \mathrm{H}]=0.03$, but the $\mathrm{H} 2 \mathrm{O}$ index suggests a spectral type near M1. Thus, the value for $[\mathrm{Fe} / \mathrm{H}]$ might be influenced by a noisy continuum at the red end of the $K$-band. Using the Newton et al. calibration, we derive $[\mathrm{Fe} / \mathrm{H}]=-0.05$. The donor star in SS Aur appears to have a solar metallicity. In Fig. 10 we present the SPEX data for SS Aur, and compare it to Gl 388, for which the metallicity calculation gives $[\mathrm{Fe} / \mathrm{H}]=+0.18$, and indicates a spectral type of M3.4. Except for the I-band, where the continuum of SS Aur is poorly defined, the data presented in this figure for both objects have been continuum divided. The CO region of the spectrum for SS Aur has low S/N, but suggests that the features are similar in strength to that of the M3V template.

The normality of the CO features is confirmed with the $K$-band spectrum of SS Aur obtained with NIRSPEC (Fig. 11). Except for the slightly bluer continuum of SS Aur, the M3V template is an excellent match for SS Aur. The metallicity analysis using the NIRSPEC data gives $[\mathrm{Fe} / \mathrm{H}]=-0.13$, and a spectral type of M2.7. It is clear that SS Aur

was in a higher state of activity when observed with NIRSPEC than with SPEX, as the He I line at $2.06 \mu \mathrm{m}$ is much stronger, and the donor star absorption features at $2.1 \mu \mathrm{m}$ are 
corrupted by He I emission in the NIRSPEC data. The AAVSO database indicates that SS Aur went into outburst six days after the Keck observations. For the SPEX observations, SS Aur had just returned to quiescence from an outburst that had its maximum three weeks prior.

The Ca I lines near $1.98 \mu \mathrm{m}$ appear to be very weak in the SPEX data for SS Aur, but the telluric correction is almost certainly responsible for this deviation. The Mg I features at 1.5 and $1.7 \mu \mathrm{m}$ also appear to be weaker in SS Aur than the template, but the depth of those lines is altered by emission lines of He I. Potassium, aluminum, and manganese appear to have solar abundances. Note that the Ca II triplet in the $I$-band is in emission, and superposed on the $\mathrm{TiO}$ bandhead at $8432 \AA$. We conclude that the donor star in SS Aur is completely consistent with a near-solar metallicity dwarf of spectral type M3.

Given the normality of the donor star in SS Aur, we can estimate a distance to this system. With $K=12.00$, and $\mathrm{M}_{K_{\mathrm{M} 3}}=6.5, d=126$ pc. The $H S T$ parallax gave $d=167$ \pm 9 pc. The spectroscopic parallax is not in close agreement with the astrometric parallax. For that to occur, requires that the secondary in SS Aur to actually have the luminosity of an $\mathrm{M} 2 \mathrm{~V}$ (the spectroscopic distance is then $d=155 \mathrm{pc}$ ). We will encounter a similar issue for U Gem.

\subsubsection{U Geminorum}

U Gem has an orbital period that is very similar to SS Aur, but we will find that it has a very different secondary star. U Gem was observed using TripleSpec at APO over a time interval of $8.3 \mathrm{hrs}$, with a 30 minute break two thirds of the way through to obtain telluric standard data, as well as observe the M-type star GJ 402. Thus, our data set spanned almost two complete orbital periods. The Triplespectool routine uses night sky emission 
lines on the exposed frames for wavelength calibration. We determined a wavelength solution for each pair of spectra, resulting in a very precise calibration of the data.

The TripleSpec data for U Gem are presented in Fig. 12. As first noted by Harrison et al. (2000), the CO features are extremely weak. The metallicity analysis leads to $[\mathrm{Fe} / \mathrm{H}]=$ +0.05 , and a spectral type of M3.9. Given the weak CO features, we use the Newton et al. relationship to find $[\mathrm{Fe} / \mathrm{H}]=+0.20$. In Fig. 12 we also plot the data for GJ 402 obtained on the same night, with the same instrument. Published spectral types for this star range from M3V to M5V. The metallicity analysis gives a spectral type of $\mathrm{M} 3.8$, and $[\mathrm{Fe} / \mathrm{H}]=$ +0.27 . Thus, it should provide an excellent template for U Gem. As shown in Fig. 12, the match between $\mathrm{U}$ Gem and GJ 402 in the wavelength interval $0.94 \leq \lambda \leq 1.34 \mu \mathrm{m}$ is very good. The only issue in this bandpass is the poor $\mathrm{S} / \mathrm{N}$ of the spectrum of GJ 402 through the telluric feature at $1.112 \mu \mathrm{m}$. The FeH feature at $0.99 \mu \mathrm{m}$ shows that they both have similar metallicities. The Mg I, K I, and Al I features all overlap.

The two spectra do not match as well in the $H$-band, with the middle sections of the continuum of U Gem well above that of GJ 402. Naively, one would assume this deviation is due to H I emission from the Brackett series, since the Doppler correction for the secondary's motion makes the H I lines appear very broad (FWHM $\sim 1400 \mathrm{~km} \mathrm{~s}^{-1}$ ); but this explanation is inadequate. First, the influence of the two strongest lines of the Bracket series in the $H$-band, $\operatorname{Br} 10(\lambda 1.74 \mu \mathrm{m})$ and $\operatorname{Br} 11(\lambda 1.68 \mu \mathrm{m})$, on the spectrum of $U$ Gem can just be discerned, as they fill-in some of the absorption features of the M dwarf. They would have to be extremely broad (and much stronger) to create an excess in the continuum between these two wavelengths. This would also require broader H I emission lines in the $H$-band than seen in either the $J$ - or $K$-bands. Secondly, adding in a strong Brackett continuum at $\lambda<1.6 \mu \mathrm{m}$ should raise the blue end of the $H$-band above that of an M4V, and this is not seen. The excess in the $H$-band is not due to $\mathrm{H}$ I emission. 
Suspiciously, the excess in the $H$-band is centered near $1.65 \mu \mathrm{m}$, the location of the peak of the $\mathrm{H}^{-}$opacity minimum. As discussed in Wing \& Jørgensen (2003), and displayed in Fig. 13, lower gravity giants have larger $\mathrm{H}^{-}$opacity "bumps" in the $H$-band, as compared to higher gravity dwarfs. In Fig. 14, we plot the $H$-band spectra of U Gem, GJ 402, and that of HD4408, an M4III. The excess due to $\mathrm{H}^{-}$is obvious in the red giant spectrum. The strong and broad absorption due to water vapor seen in the dwarfs at the red end of the $H$-band, is weaker in the red giant. The water vapor features in red giants are much weaker than those of dwarfs with the same effective temperature. Thus, the most obvious explanation for the peculiarity of the $H$-band spectrum is that the secondary of $\mathrm{U}$ Gem has an apparent gravity that is much lower than that of GJ 402 .

The Na I doublet in the $K$-band was easily seen in the individual spectra, and we have used IRAF to individually measure the positions of each of the lines of the doublet to allow us to construct the radial velocity curve shown in Fig. 15. $\chi^{2}$ analysis of sinewaves with various amplitudes fit to these data finds a semi-amplitude of $\mathrm{K}_{2}=310 \pm 10 \mathrm{~km} \mathrm{~s}^{-1}$. This result agrees with the value of $309 \pm 3 \mathrm{~km} \mathrm{~s}^{-1}$ found by Friend et al. (1990), and $\mathrm{K}_{2}=302$ $\mathrm{km} \mathrm{s}^{-1}$ found by Naylor et al. (2005).

Naylor et al. attempted to determine the $v \sin i$ rotation rate of the secondary, and depending on the template they used, found values from $96 \mathrm{~km} \mathrm{~s}^{-1}$ (with an M5.5V), to 142 $\mathrm{km} \mathrm{s}^{-1}$ (with an M3V). Using the M4V template GJ 402 for comparison, and working in the $J$-band ( $5^{\text {th }}$ order, a dispersion of $\Delta \lambda=1.7 \AA$ ), we find a best fit value for $v \sin i=155$ $\pm 20 \mathrm{~km} \mathrm{~s}^{-1}$. This large value may result from the fact that GJ 402 has a slightly higher metallicity than U Gem. The spectral features for a higher metallicity object are going to be slightly deeper, and thus would require a larger velocity convolution to best match the observed spectrum (see Shahbaz 1998). To test this, we used SPECTRUM to generate synthetic $J$-band spectra using $\mathrm{T}_{\text {eff }}=3,200 \mathrm{~K}, \log g=4.5$, and MARCS models with $[\mathrm{Fe} / \mathrm{H}]$ 
$=0.0$ and -0.50 . Given the uncertainties in modeling $\mathrm{M}$ dwarf atmospheres we limited our analysis to $1.24 \leq \lambda \leq 1.26 \mu \mathrm{m}$ (essentially the two K I lines). A model generated using the parameters of GJ 402 reproduced these two lines. The best fit $\left(\chi^{2}\right)$ value for the rotation velocity of $\mathrm{U}$ Gem using the solar metallicity model has $v \sin i=145 \mathrm{~km} \mathrm{~s}^{-1}$, while the sub-solar metallicity model gives $v \sin i=120 \mathrm{~km} \mathrm{~s}^{-1}$.

It is obviously critical to have the appropriate metallicity template when using this technique to estimate $v \sin i$. Thus we note that, except for Gl 51 and Gl $65 \mathrm{~A}$, the late-type templates used by Naylor et al. for their vsini derivation have sub-solar metallicities. Gl 51 and Gl $65 \mathrm{~A}$ are slightly cooler $\left(\mathrm{T}_{\text {eff }}=3030 \mathrm{~K}, \simeq \mathrm{M} 5 \mathrm{~V}\right)$ than the donor in $\mathrm{U}$ Gem. The closest temperature match is GJ 213, but it has the lowest metallicity of the sample $([\mathrm{Fe} / \mathrm{H}]$ $=-0.22$, Mann et al. 2015). Convolving the spectrum of GJ 463 gave their highest $v \sin i$ estimate, but there are no measurements of $[\mathrm{Fe} / \mathrm{H}]$ for this star (though it has a similar temperature to U Gem, $\mathrm{T}_{\text {eff }}=3300 \mathrm{~K}$, Houdebine \& Mullan 2015). Given the uncertainties in this process, our result for $v \sin i$ is certainly consistent with the conclusions of Naylor et al. This confirms their result that $\mathrm{U}$ Gem has a massive white dwarf, $\mathrm{M}_{1} \simeq 1.2 \mathrm{M}_{\odot}$. The mass of the secondary that they derive, $\mathrm{M}_{2}=0.44 \mathrm{M}_{\odot}$, is similar to those of main sequence M2/M3 dwarfs.

Given the parameters of the solution found by Naylor et al. (2005), the surface gravity of the secondary in $\mathrm{U}$ Gem would be $\log g=4.8$. A value similar to those of normal M dwarfs (cf., Fernandez et al. 2009). Our larger values of $\mathrm{K}_{2}$ and $v \sin i$ result in $\mathrm{M}_{2}=0.42$ $\mathrm{M}_{\odot}$, and an equatorial radius of $\mathrm{R}_{2}=0.58 \mathrm{R}_{\odot}$, leading to $\log g=4.5$. This would imply a gravity at the equator that is one half that of a typical $\mathrm{M}$ dwarf. Applying the relations in Warner (1995) to U Gem, the equatorial radius is 1.64 times larger than the volume radius " $\mathrm{R}_{\mathrm{L}}$ " of the Roche lobe (the radius of the equivalent sphere). Thus, using our result, the surface gravity of the equivalent sphere is then $\log g=5.0$. Since U Gem has a large 
inclination $\left(i=69^{\circ}\right)$, the equatorial regions of the secondary will dominate the spectrum. Since these regions are further away from the center of the star, they will be cooler, leading to a cooler observed spectral type. This is, of course, the effect of "gravity darkening" found for rotating stars, first quantified by von Zeipel (1924). We explore whether we can reconcile the results for $U$ Gem with the expected levels of gravity darkening in $§ 6.2$.

This inference would resolve the paradox from the HST parallax measurement, where the luminosity of the secondary star was equivalent to an $\mathrm{M} 2 \mathrm{~V}$, but the photometry and spectroscopy indicated an M4V (Harrison et al. 2000). It also explains the "too cool for comfort" finding of Friend et al (1990), where the observed spectral type was later than expected if the donor was to obey the main sequence mass-radius relationship. A more robust value of $v \sin i$ for $\mathrm{U}$ Gem is required to confirm this result.

To attempt to quantify the carbon deficit, we have continuum divided the $K$-band spectra of both U Gem and GJ 402 to remove the water vapor features. In the process, we also remove much of the broader absorption due to CO, and thus these features will also be weakened by this process, resulting in an artificial deficit in carbon. We have used kmoog to generate model spectra to determine a relative value for the carbon deficit between $\mathrm{U}$ Gem and GJ 402. As noted above, the line lists and atmosphere models for $\mathrm{M}$ dwarfs are poorly known, so the following process has considerable uncertainty. kmoog was run with $\mathrm{T}_{\text {eff }}=3200 \mathrm{~K},[\mathrm{Fe} / \mathrm{H}]=0$, and $g=4.5$, using a MARCS atmosphere model, over a wide range of carbon abundances $(0.0 \leq[\mathrm{C} / \mathrm{Fe}] \leq-2.5)$. The best fit results are shown in Fig. 16. The Na I doublet and Ca I triplet in the synthetic spectra do a reasonable job fitting the observations. We find that we need to artificially have a carbon abundance of $[\mathrm{C} / \mathrm{Fe}]=$ -0.5 to match the features in GJ 402, while $\mathrm{U}$ Gem has to have $[\mathrm{C} / \mathrm{Fe}]=-1.5$. Thus, we estimate that the $\mathrm{C}$ abundance for the donor star in $\mathrm{U}$ Gem is $\sim 10 \%$ of solar, in agreement with the value derived from UV spectroscopy of the white dwarf primary (Long \& Gilliland 
1999).

One additional insight from this modeling process are the strengths of the ${ }^{13} \mathrm{CO}$ features in U Gem. Harrison et al. (2005b) commented on the ease at which the ${ }^{13} \mathrm{CO}_{(2,0)}$ $(\lambda 2.345 \mu \mathrm{m})$ feature was seen in their SPEX data for U Gem, given the weakness of the ${ }^{12} \mathrm{CO}$ features. In Fig. 16, we identify the locations of the ${ }^{12} \mathrm{CO}$ bandheads with solid lines, and the ${ }^{13} \mathrm{CO}$ bandheads with dashed lines. At the normal isotopic ratio of ${ }^{12} \mathrm{CO} /{ }^{13} \mathrm{CO}=$ 89 used in the GJ 402 model, only the ${ }^{13} \mathrm{CO}_{(2,0)}$ bandhead at $2.345 \mu \mathrm{m}$ is visible. In U Gem, this feature is of similar strength to the ${ }^{12} \mathrm{CO}_{(2,0)}$ bandhead at $2.294 \mu \mathrm{m}$ ! The ${ }^{13} \mathrm{CO}_{(3,1)}$ bandhead at $2.374 \mu \mathrm{m}$ falls at the position of a strong telluric feature that is always difficult to remove. The next ${ }^{13} \mathrm{CO}$ bandhead at $2.403 \mu \mathrm{m}\left({ }^{13} \mathrm{CO}_{(4,2)}\right)$, however, falls in a cleaner region, and is clearly present in U Gem. In our synthetic spectrum for U Gem we used an isotopic ratio of ${ }^{12} \mathrm{CO} /{ }^{13} \mathrm{CO}=1$. Such a ratio is beyond the maximum ratio (4) that can be achieved by the CNO cycle. With the uncertainties inherent to modeling M dwarf spectra, a more precise value will require better synthetic data. We conclude that ${ }^{13} \mathrm{C}$ is highly overabundant in the donor star of U Gem.

Given the caveat noted earlier, assuming the results by Veyette et al. (2016) are correct, the actual value for $[\mathrm{Fe} / \mathrm{H}]$ for $\mathrm{U}$ Gem is much higher than derived from the metallicity relationships due to the lack of carbon in its spectrum. If we assume the $\mathrm{O}$ abundance is normal in $\mathrm{U}$ Gem (leading to $\mathrm{C} / \mathrm{O}=0.055$ ), then we have underestimated $[\mathrm{Fe} / \mathrm{H}]$ by $\sim-0.3$. If this offset is true, $\mathrm{U}$ Gem has a very high, super-solar metallicity of $[\mathrm{Fe} / \mathrm{H}] \simeq 0.35$, bringing it into closer agreement with GJ 402 . 


\subsubsection{WW Ceti}

The long-term AAVSO light curve of WW Cet is unusual, showing frequent DNe outbursts, periods of intermediate brightness that suggest Z Cam-like behavior, but also low states like VY Scl. Ringwald et al. (1996) find that the behavior of WW Cet is not consistent with a Z Cam classification, nor are its low states like the VY Scl stars. The orbital period of $\mathrm{WW}$ Cet, $\mathrm{P}_{\text {orb }}=4.22 \mathrm{hr}$, is just a bit shorter than that of SS Aur and U Gem. Thus, the odd behavior of WW Cet is puzzling. The SPEX data for WW Cet are presented in Fig. 17. The metallicity relationship gives a spectral type of $\sim \mathrm{M} 0 \mathrm{~V}$, and $[\mathrm{Fe} / \mathrm{H}]=-0.17$, suggesting that noise at the red end of the $K$-band might be causing issues with the $\mathrm{H}_{2} \mathrm{O}$ index. However, input of the same $\mathrm{H}_{2} \mathrm{O}$ index into the metallicity relationship as that for $\mathrm{U}$ Gem leads to an identical value of $[\mathrm{Fe} / \mathrm{H}]$. Using only the $\mathrm{Na} I$ equivalent width we derive $[\mathrm{Fe} / \mathrm{H}]=-0.34$. It appears certain that the secondary in WW Cet has a subsolar metallicity.

The IRTF Spectral Library fortunately has spectra for a number of sub-solar metallicity $\mathrm{M}$ dwarfs in the spectral range $\mathrm{M} 2 \mathrm{~V}$ to $\mathrm{M} 4 \mathrm{~V}$. We compared the spectra of each of these to WW Cet, and find that the best fit results from the M2.5V dwarf Gl 381. The continuum of WW Cet is slightly bluer than this star, with the excess increasing to the blue, suggesting the presence of emission from the white dwarf + accretion disk. The metallicity relationship gives $[\mathrm{Fe} / \mathrm{H}]=-0.06$ for $\mathrm{Gl} 381$, while Gaidos et al. (2014) find $[\mathrm{Fe} / \mathrm{H}]=-0.07$. All of

the atomic absorption features in the template are stronger than their counterparts in WW Cet. This would be expected given the lower metallicity computed for WW Cet. Even accounting for the difference in metallicity, the CO features in WW Cet are weaker than the template (note the excess at the red end of the $K$-band). Sion et al. (2006) found that the carbon abundance of the white dwarf was 10\% solar, and silicon was $50 \%$ solar. Clearly, the deficit of carbon is not as extreme in WW Cet as it is in U Gem. Building on the 
experience gained above, we estimate that the carbon abundance of the donor in WW Cet is $[\mathrm{C} / \mathrm{Fe}] \geq-0.3$.

\subsubsection{LS Pegasi}

LS Peg, $\mathrm{P}_{\text {orb }}=4.19 \mathrm{hr}$, had previously been classified as an SW Sex star (Taylor et al. 1999). Recently, however, it has been re-classified as an IP by Ramsay et al. (2008). They believe that the lack of an X-ray beat period, a phenomenon common to IPs, is due to the fact that the magnetic and spin axis of the white dwarf are aligned. The cross-dispersed

spectrum of LS Peg is shown in Fig. 18. Except, perhaps, for the Na I doublet at $0.82 \mu \mathrm{m}$, no absorption features from the secondary are evident. The spectrum is dominated by $\mathrm{H}$ I and He I emission. In addition, there are strong CO emission features. He II at $2.18 \mu \mathrm{m}$ appears to be present, and a small peak on the red wing of H I Br11 might be [Fe II]. It is also clear that $\mathrm{Ca}$ II is in emission, as two of the members of the high order H I Paschen series (Pa13 and Pa15) are elevated above their expected levels. Of the eight IPs for which we have obtained NIR spectra, none of them have had CO in emission (Harrison et al. 2007a, 2010). It is now apparent, however, that Ca II emission was present in the spectrum of V603 Aql (Fig. 4 in Harrison et al. 2007a). The same set of unidentified emission lines in the spectrum of LS Peg (see Fig. 18), were also present in the spectrum of V603 Aql.

\subsubsection{KT Persei}

KT Per is a Z Cam-type dwarf nova with a period of $3.90 \mathrm{hr}$ (Thorstensen \& Ringwald 1997). They derived a spectral type of M3 for the secondary star. The IRTF data for KT Per are presented in Fig. 19. The shorter orbital period, as compared to U Gem and SS Aur, would lead to the expectation of a mid-M spectral type for the donor star. The 
metallicity analysis gives $[\mathrm{Fe} / \mathrm{H}]=-0.53$, and a spectral type of M1. The Na I relation gives $[\mathrm{Fe} / \mathrm{H}]=-0.96$, though if the spectral type of the secondary was truly an M1V, this could possibly be erroneous (see Newton et al. 2014). Unfortunately, the IRTF Spectral Library does not have a template with similar properties. The closest match is Gl 806 for which the metallicity relation gives $[\mathrm{Fe} / \mathrm{H}]=-0.08$, and a spectral type of M2.2. Mann et al. (2015) lists $\mathrm{Gl} 806$ as an M2.0 with $[\mathrm{Fe} / \mathrm{H}]=-0.15$. It is clear that the continuum of Gl 806 closely matches that of KT Per in the $K$-band. At shorter wavelengths, there is an increasing contribution from the white dwarf + accretion disk. It is obvious that all of the spectral absorption features in the spectrum of KT Per are much weaker than those in Gl 806. This includes the water vapor features, confirming the very low metallicity estimate. Given this result, the CO features then appear to be normal. The Ca II triplet lines in the $I$-band are in absorption for the donor star.

Given the low level of contamination in the $K$-band, it is possible to estimate a distance to KT Per if we assume a normal M2V. The 2MASS catalog has $K=12.63$. To insure that this measurement reflects true quiescence, we consulted the NEOWISE single exposure source catalog ${ }^{10}$, There are 83 measurements for KT Per in this database, and they show that the faintest it gets is $\mathrm{W} 1(3.6 \mu \mathrm{m})=12.25$. For a normal M2V, $(K-\mathrm{W} 1)$ $=0.15$ (Pecaut \& Mamajek 2013). KT Per is slightly redder than expected, but clearly the 2MASS value must be close to quiescence. Assuming $\mathrm{M}_{K}=6.05$, gives $d=207 \mathrm{pc}$, just within the error bars on the distance derived from its parallax: $d=180_{-28}^{+36} \mathrm{pc}$ (Thorstensen et al. 2008). Thorstensen et al. note that there is a common proper motion companion to KT Per which has a mid-K spectral type. Using the data in the 2MASS catalog for this object, the NIR colors are consistent with a $\mathrm{K} 5 \mathrm{~V}$, and the resulting distance to this companion is 299 pc. There is either significant contamination to the $K$-band luminosity

\footnotetext{
${ }^{10}$ http://irsa.ipac.caltech.edu/cgi-bin/Gator/nph-scan?projshort=WISE\&mission=irsa
} 
of KT Per, or else the secondary is 0.8 mag more luminous than a main sequence M2V, having the absolute magnitude of an M1V. The 2MASS colors suggest the contamination is low, and this is supported by the ease at which Thorstensen \& Ringwald detected the donor in optical spectra. Unfortunately the orbital inclination for KT Per is unconstrained. If we assume an offset of one spectral subtype due to inclination effects, the spectral type and distance can be reconciled if $i \sim 40^{\circ}$.

\subsubsection{TT Arietis}

Our final short period CV with cross-dispersed SPEX data is TT Ari, $\mathrm{P}_{\text {orb }}=3.30$ hr. The data are presented in Fig. 20, and show no absorption features from a late-type secondary star. However, the Ca II triplet is in emission, and presumably originates on the donor. If we assume that the secondary in TT Ari has a spectral type of M4, we can add a hot $\left(\mathrm{T}_{\text {eff }}=10,000 \mathrm{~K}\right)$ blackbody continuum source until the spectral slope in the $K$-band is reproduced. From this iterative process, we find that the blackbody source has $3.5 \times$ the luminosity of the secondary in the $K$-band. Given that TT Ari has $K_{2 \mathrm{MASS}}=10.88$, and an $\mathrm{M} 4 \mathrm{~V}$ has $\mathrm{M}_{K}=7.43$, the distance to TT Ari is $\mathrm{d} \sim 104 \mathrm{pc}$.

\subsection{Metallicity Analysis for CVs with Expected M Dwarf Donors Using $K$-band Data Only}

\subsubsection{TW Virginis}

TW Vir has a period very similar to SS Aur and U Gem. The SPEX data were presented in Harrison et al. (2005a). TW Vir has a blue continuum, with unusually strong $\mathrm{Na}$ I doublet and Ca I triplet features. Running the metallicity analysis for this spectrum finds a spectral type of $\mathrm{M} 0 \mathrm{~V}$, and $[\mathrm{Fe} / \mathrm{H}]=-0.3$. Clearly, this result is compromised 
by the significant contamination of the secondary by the white dwarf and accretion disk. Subtracting a blackbody with $\mathrm{T}_{\text {eff }}=10,000 \mathrm{~K}$ that has $67 \%$ of the $K$-band flux results in a continuum that is consistent with an $\mathrm{M} 1 \mathrm{~V}$, while subtraction of a hot blackbody that supplies $77 \%$ of the $K$-band flux results in a spectrum whose continuum matches that of an M4V. The metallicity analysis on the subtracted spectra yield $[\mathrm{Fe} / \mathrm{H}]=0.56$, and 1.2 , respectively. The M1V dwarf HD 42581 in the IRTF spectral library has $[\mathrm{Fe} / \mathrm{H}]=0.2$, while the M4.5V dwarf Gl $268 \mathrm{AB}$ from the same source has $[\mathrm{Fe} / \mathrm{H}]=0.26$. It is clear that both $\mathrm{Na}$ I doublets, and the Ca I triplet in the subtracted TW Vir spectra are much stronger than those in either HD42581 or Gl 268AB.

Obviously, such high values of $[\mathrm{Fe} / \mathrm{H}]$ for TW Vir are not reasonable, being as high, or higher, than seen anywhere in the Galaxy. Using the inferences of Veyette et al. (2016), to bring the metallicity of TW Vir in line with normal stars would require an oxygen deficiency. If $[\mathrm{O} / \mathrm{Fe}]=-0.2$, the derived abundance for TW Vir would be reduced by $\Delta[\mathrm{Fe} / \mathrm{H}]=-0.6$. It is not clear how to produce such a deficit without it being pre-existing. As shown by Veyette et al., there are few such objects in the solar neighborhood. The result that we will derive for QZ Ser in the next section may provide the necessary insight for explaining this object. Clearly, however, new higher $\mathrm{S} / \mathrm{N}$ data spanning the entire NIR will be essential for further progress.

\subsection{2. $C W$ Monocerotis}

CW Mon is a typical DNe with an orbital period of $4.238 \mathrm{hr}$, and exhibits grazing eclipses (Kato et al. 2003). CW Mon has a significant X-ray luminosity (Verbunt et al. 1997), suggesting that it might be an IP. Kato et al. found a possible white dwarf spin period that would aid in this classification, but such a periodicity only appears to show up during outbursts. CW Mon was observed with NIRSPEC, and the spectrum is shown in 
Fig. 21. The metallicity analysis indicates a spectral type of $\mathrm{M} 2.3$, and $[\mathrm{Fe} / \mathrm{H}]=-0.23$. The IRTF template Gl 806 has a spectral type of $\mathrm{M} 2.2 \mathrm{~V}$, and $[\mathrm{Fe} / \mathrm{H}]=-0.15$, and proves to be an excellent match for CW Mon. There are no abundance anomalies, though the continuum of Gl 806 appears to be slightly bluer than CW Mon, indicating a slightly later spectral type for the donor. The IRTF spectral template star Gl $381(\mathrm{M} 2.9,[\mathrm{Fe} / \mathrm{H}]=$ -0.06) provides a much better match to the continuum, but not to the depth of the Na I and Ca I absorption features.

Verbunt et al. list a distance for CW Mon of 290 pc, though the origin of this "adopted distance" is not obvious. In the 2MASS catalog, CW Mon has $(J-H)=0.53,(H-K)$ $=0.32$, and $K=13.02$, roughly consistent with an M2/3. The AAVSO database shows that CW Mon was at minimum light during the epoch of the 2MASS observations. If the secondary star is a normal M2V, the distance to CW Mon is $\mathrm{d}=247 \mathrm{pc}$. If the " $\mathrm{U}$ Gem effect" is universal, the large inclination of CW Mon, $i=65^{\circ}$ (Szkody \& Mateo 1986), would suggest a true spectral type closer to M1V, giving $d=311 \mathrm{pc}$, closer to the Verbunt et al. estimate. As noted by Kato et al., at that distance, the visual maxima of CW Mon are nearly one magnitude less luminous than expected.

\subsection{3. $Y Y / D O$ Draconis}

YY/DO Dra (see Patterson \& Eisenman 1987 for comments on its designation) is an IP with an orbital period of $3.969 \mathrm{hr}$, and a spin period of $264.6 \mathrm{~s}$ (Patterson \& Szkody 1993). Like a small subset of its brethren (see Szkody et al. 2002), it shows infrequent dwarf novae outbursts of short duration. Metallicity analysis on these data find a spectral type

of $\mathrm{M} 1.8$, and $[\mathrm{Fe} / \mathrm{H}]=-0.20$. The actual continuum of YY/DO Dra is better matched by the M2.5V template Gl $381(\mathrm{M} 2.9,[\mathrm{Fe} / \mathrm{H}]=-0.06)$. The NIRSPEC data of YY/DO Dra are shown in Fig. 24, along with the spectrum of Gl 381. Clearly, the absorption lines in 
the template are stronger than those seen in YY/DO Dra, consistent with their difference in metallicity. It is also obvious, however, that the CO features in YY/DO Dra are weaker than those of the template. We estimate that $[\mathrm{C} / \mathrm{Fe}] \leq-0.3$.

As noted in the discussion of U Gem, weaker CO features should lead to an underestimation of the metallicity if the conclusions of Veyette et al. (2016) are correct (and we assign the entire deficit to carbon). In the case of YY/DO Dra, it clearly has weak $\mathrm{CO}$, but the derived value of $[\mathrm{Fe} / \mathrm{H}]$ and the spectral comparison are consistent with it being lower metallicity than that of the template. If $[\mathrm{C} / \mathrm{Fe}]=-0.2$, the results of Veyette et

al. would lead to the derived metallicity being underestimated by $\Delta[\mathrm{Fe} / \mathrm{H}]=-0.15$. This offset would imply that both objects have the same metallicities, which is clearly not true.

\subsubsection{UU Aquilae}

The spectrum of UU Aql was discussed in Harrison et al. (2005a) where a spectral type of M2 was found to match the continuum, but not the absorption features. Especially noteworthy was the absence of CO absorption. The metallicity analysis suggests a spectral type of $\mathrm{K} 8$, and $[\mathrm{Fe} / \mathrm{H}]=-0.34$. The NaI-only relation of Newton et al. gives $[\mathrm{Fe} / \mathrm{H}]=$ -0.98. The expectation at the orbital period of UU Aql is for a mid-M type donor. We compare the spectrum of UU Aql to the M2V template Gl 806 in Fig. 22, and it is clear that $\mathrm{CO}$ is in emission in this system. This explains the discrepant spectral type estimation from the $\mathrm{H} 2 \mathrm{O}$ index. The weakness of the blue Ca I lines, and the Na I doublet in UU Aql, vis-á-vis Gl 806, suggests that it has a much lower metallicity. If we use the H2O index of Gl 806 for UU Aql, the Mann et al. (2013) relation gives $[\mathrm{Fe} / \mathrm{H}]=-0.72$. Even with the uncertainty induced by CO emission, the secondary star of UU Aql appears to have an extremely low metallicity. 
In the 2MASS catalog, UU Aql has $(J-H)=0.69,(H-K)=0.24$, and $K=13.33$, consistent with an M2V. There are no AAVSO data for the epoch of these observations, but they occurred halfway between two maxima, suggesting that UU Aql was near quiescence. If we assume a normal M2V donor, this suggests a distance of $\mathrm{d}=286 \mathrm{pc}$. Sion et al. (2007) found that from modeling FUSE observations, they could not settle on a distance for UU Aql, with both $\mathrm{d}=150 \mathrm{pc}$, and $\mathrm{d}=300 \mathrm{pc}$ producing nearly identical results. A model of theirs with the silicon and carbon abundances at $10 \%$ the solar value, however, gave a distance of 314 pc. Perhaps dramatically lowering the global abundances in their white dwarf/accretion disk models might lead to more robust fits. It is important to note that Woolf \& West (2012) show that low metallicity M dwarfs are less luminous than more metal-rich stars. For $[\mathrm{Fe} / \mathrm{H}]=-0.5$, they found $\Delta \mathrm{M}_{V}=+0.53$. If that offset is applied here, the distance to $\mathrm{UU} \mathrm{Aql}$ is reduced to $224 \mathrm{pc}$.

\subsubsection{CN Orionis}

The orbital period of CN Ori is about $30 \mathrm{~s}$ shorter than UU Aql, so one would expect them to appear similar. The NIRSPEC spectrum for CN Ori is shown in Fig. 23, where we compare it to Gl 806. The continua of the two sources match quite well. The metallicity analysis gives the spectral type as $\mathrm{M} 1.1$, and $[\mathrm{Fe} / \mathrm{H}]=-0.49$. While more subtle than seen in $\mathrm{UU}$ Aql, $\mathrm{CO}$ emission is clearly present in the spectrum of $\mathrm{CN}$ Ori, so the spectral type calculated from the $\mathrm{H} 2 \mathrm{O}$ index will be corrupted. If we again assume the $\mathrm{H} 2 \mathrm{O}$ index of Gl 806, we find $[\mathrm{Fe} / \mathrm{H}]=-0.71$ for $\mathrm{CN}$ Ori. Essentially identical to UU Aql. H\&H modeled several CO emission scenarios and showed that when the velocity width of the emission is low, the underlying CO absorption features of the secondary star appear much narrower, with small blue emission wings. The red end of the model spectrum at the bottom of their Fig. 21 looks nearly identical to that of CN Ori (see also Fig. 9). As we found for UU Aql, 
the main absorption features in the spectrum of CN Ori are much, much weaker than their counterparts in Gl 806. This supports the very low metallicity calculation.

Verbunt et al. (1997) lists a distance of 295 pc. The 2MASS colors of CN Ori are $(J-H)=0.50,(H-K)=0.20$, and $K=13.10$. The AAVSO light curve data shows that the 2MASS observations fell in-between two outbursts. The $(J-H)$ color is slightly bluer than an M2V by $0.1 \mathrm{mag}$, while $(H-K)$ matches such an object exactly. The estimated distance is 257 pc. Again, due to the low metallicity of the secondary star, this may be an overestimate.

\subsubsection{IP Pegasi}

IP Peg is an eclipsing CV with an orbital period of $\mathrm{P}_{\text {orb }}=3.797 \mathrm{hr}$. Ribeiro et al. (2007) modeled $J H K$ light curves of IP Peg in quiescence and found $i=84.5, \mathrm{~T}_{2}=3100 \pm$ $500 \mathrm{~K}$, and a distance of 115 pc. The NIRSPEC data for IP Peg is shown in Fig. 25, where it is compared to the MOV template HD 19305. Metallicity analysis on the NIRSPEC data finds a spectral type of $\mathrm{M} 0.9$, and $[\mathrm{Fe} / \mathrm{H}]=0.18$. The same analysis on HD 19305 derives a spectral type of $\mathrm{M} 0.7$, and $[\mathrm{Fe} / \mathrm{H}]=0.02$. Thus, HD 19305 should provide a reasonable comparison, and this is confirmed in Fig. 25. Except for the Al I doublet at 2.1 $\mu \mathrm{m}$, the main atomic absorption features in IP Peg are slightly stronger than the template, consistent with the metallicity difference between the two objects. The Al I features might be compromised by He I emission, though the always much stronger He I emission line at $2.06 \mu \mathrm{m}$ is quite weak in IP Peg. The CO features in IP Peg appear to be marginally weaker than the template, though the telluric correction at the red end of the NIRSPEC spectrum is not of the highest quality.

As shown in Table 1, the secondary of IP Peg was at inferior conjunction at the time 
of the NIRSPEC observations, and the AAVSO light curve database shows that the system was quiescent (though it would go into outburst 10 days later). Thus, it is somewhat surprising that we obtain a much earlier spectral type than found by Ribeiro et al. ( M3.5). 2MASS data was obtained at $\phi=0.35$, and with $(J-K)=0.88$, suggests an M2V. With $K_{2 \mathrm{MASS}}=11.72$, and assuming the secondary is a normal M1V, the distance to IP Peg is $172 \mathrm{pc}$.

\subsubsection{AM Herculis}

AM Herculis is the proto-type for the polars, and has an orbital period of $3.09 \mathrm{hr}$. As shown in Campbell et al. (2008), AM Her has strong cyclotron emission in the $K$-band during much of its orbit. The SPEX data for AM Her presented in Harrison et al. (2005a) were obtained during a polar high state, and near inferior conjunction, when the cyclotron emission is at its strongest. Campbell et al. observed AM Her over a full orbit during a low state using the low resolution "prism" mode of SPEX. During that time they found a spectral type of M5V. The metallicity analysis on the higher resolution SPEX data in Harrison et al. (2005a) finds a spectral type of $\mathrm{M} 1.2$, and $[\mathrm{Fe} / \mathrm{H}]=-0.25$. To attempt to determine whether this metallicity is at all reliable, we have continuum-divided the moderate resolution SPEX data for AM Her to compare with the spectral templates. The M4V template HIP9291 (H2O index gives M4.7) has $[\mathrm{Fe} / \mathrm{H}]=0.15$, while Gl 213 (M4.6) has $[\mathrm{Fe} / \mathrm{H}]=-0.31$. The resulting match between the spectra of Gl 213 and AM Her is excellent, except that the CO features in AM Her are weaker than those in the template. Running the metallicity routine on the low resolution SPEX data from Campbell et al. (2008) at phase 0.5, when the cyclotron emission is near its minimum, we derive a spectral type of M5.0, and $[\mathrm{Fe} / \mathrm{H}]=-0.44$. It is possible that SPEX prism mode spectroscopy is of insufficient resolution for these routines to work reliably, however, the results using such 
data are consistent with the those derived using the other techniques. We conclude that secondary star in AM Her has a low metallicity, and probably a carbon deficit. Higher spectral resolution observations during a low state, and at phases of low cyclotron emission, are needed to confirm this result.

Thorstensen (2003) obtained a parallax for AM Her that leads to a distance of $79_{-6}^{+8}$ pc. An M5V at this distance would have $K=13.00$, while an M4V would have $K=11.9$. Campbell et al. (2008) present a $J H K$ light curves for AM Her, and from their ellipsoidal model, $\langle K\rangle=11.45$, and $i=50^{\circ}$. The donor in AM Her has the luminosity of an M3.5V, 1.5 subtypes earlier than that derived from spectroscopy.

\subsubsection{The Polars from Harrison et al. $2005 b$}

We lump together the results for the polars discussed in Harrison et al. (2005b) to examine their similarities. There are four short period polars that were observed using NIRSPEC on Keck: AR UMa, MR Ser, ST LMi, and VV Pup. They also observed the longer period low accretion rate polar MQ Dra (= SDSS1553+5516). None of the donor stars in these systems appeared to be unusual in any way, with normal carbon abundances for their spectral types. As shown in Table 3 , the secondaries in all four short period polars have subsolar metallicities, while MQ Dra has a super-solar metallicity.

Harrison \& Campbell (2015) discussed the field strengths for the four short period polars, and examined/modeled WISE mid-IR light curve data for these systems. AR UMa has a large field strength, thus it has no cyclotron emission in the NIR, and its $K$-band spectrum will be uncorrupted from non-thermal emission sources. The spectral type from the $\mathrm{H} 2 \mathrm{O}$ index, M4.5, agrees with the classification in Harrison et al. (2005b). It is likely that there is weak cyclotron emission from the $n=2$ harmonic in the $K$-band for MR Ser. 
In modeling its infrared light curve data, Harrison \& Campbell (2015) used a donor with T $=2400 \mathrm{~K}(\sim \mathrm{M} 8)$. This contrasts with the M4.5 type derived from the metallicity analysis. The NIRSPEC and SPEX data for MR Ser (see Fig. 2 in Harrison et al. 2005b) show no evidence for the short or long wavelength turnovers due to water vapor that would be expected from a late type M dwarf. Harrison \& Campbell (2015) present a phase-resolved set of SPEX prism spectra for MR Ser, but their data did not cover a full orbit, and at no phase does the cyclotron emission in this system drop to very low levels like that in AM Her.

ST LMi can have strong cyclotron emission in the $K$-band during part of its orbit; fortunately the NIRSPEC observations missed those phases. The spectral type derived from the metallicity analysis, M6.1, agrees with the M6V classification from Campbell et al. (2008). Harrison \& Campbell find that the field strengths of the two poles in VV Pup are $\mathrm{B}=57$ and $33 \mathrm{MG}$, thus there are no cyclotron harmonics in the $K$-band. Harrison et al. (2005b) derived a spectral type of M7, in agreement with that from the H2O index. Infrared light curves of MQ Dra are presented in Szkody et al. (2008) and show that there is no evidence for cyclotron emission in the $K$-band. Thus, the metallicity analysis for this object should be robust.

Thorstensen et al. (2008) have published parallaxes for four of the five polars discussed here. For VV Pup, they found $d=124_{-14}^{+17} \mathrm{pc}$, AR UMa has $d=86_{-8}^{+9} \mathrm{pc}$, MR Ser has $d=$ $126_{-12}^{+14} \mathrm{pc}$, and MQ Dra has $d=162_{-21}^{+27}$. From Harrison \& Campbell $(2015),\left\langle K_{\mathrm{AR} \text { UMa }}\right\rangle=$ 13.2, and $\left\langle K_{\mathrm{MR} \text { Ser }}\right\rangle=14.0$. From Szkody et al. (2008), $\left\langle K_{\mathrm{MQ} \text { Dra }}\right\rangle=13.7$, while Szkody \& Capps (1980) found $\left\langle K_{\mathrm{VV} \text { Pup }}\right\rangle=15.1$. The absolute $K$ magnitudes of AR UMa and MR Ser $\left(\mathrm{M}_{K}=8.5\right)$ are identical to that of an M5V, while VV Pup is a full magnitude less luminous, being more consistent with an M6V. MQ Dra has the most luminous secondary of these four objects, $\mathrm{M}_{K}=7.6$, suggesting the donor star has a luminosity slightly fainter 
than that of an M4V main sequence star. The classifications derived from their luminosities are in good agreement with those found from the spectral analysis. The donor stars in these five systems are very similar to normal main sequence stars.

\subsubsection{RZ Leonis}

The NIRSPEC data for RZ Leo have been presented in Howell et al. (2010), and Hamilton et al. (2011). RZ Leo is a short period DNe in the WZ Sge family. This subclass of CVs have infrequent, but large amplitude outbursts. Both Howell et al. and Hamilton et al. classify the secondary spectral type as M4. We have used measurements of the wavelength of the Na I doublet to Doppler correct the NIRSPEC data (see Fig. 26). The metallicity analysis on the resulting spectrum gives a spectral type of M3.5, and $[\mathrm{Fe} / \mathrm{H}]$ $=-0.44$. However, measurement of the $\mathrm{Na}$ I doublet and its local continuum is probably compromised by the broad H I Br $\gamma$ emission. In addition, the Ca I triplet has a much broader profile than it should. Comparison to the various late-type templates finds that Gl 268AB $(\mathrm{M} 4.8,[\mathrm{Fe} / \mathrm{H}]=0.26)$ provides an excellent match to the continuum and to both $\mathrm{Na}$ I doublets of RZ Leo. This suggests that the donor has a solar metallicity. While the CO features in RZ Leo appear to be only slightly weaker than the template, the first overtone of ${ }^{13} \mathrm{CO}$ at $2.345 \mu \mathrm{m}$ is very strong. The data are noisy, but suggest that the ${ }^{13} \mathrm{CO}_{(4,2)}$ feature at $2.403 \mu \mathrm{m}$ is also present.

It is rare for such a short period system to have such a prominent secondary. We suspect that there is contamination from a hot source that makes the continuum appear bluer than expected. If so, the spectral type of the secondary is probably later than M5, and that would imply a substantial carbon deficit. For example, if we subtract-off a 10,000 K blackbody that supplies $30 \%$ of the $K$-band flux (Fig. 26), the continuum of RZ Leo is very similar to that of GJ $1111(\mathrm{M} 6.8,[\mathrm{Fe} / \mathrm{H}]=-0.17)$. The donor in RZ Leo would then 
have a substantial subsolar abundance, and a significant carbon deficit (note, however, that ${ }^{13} \mathrm{C}$ would not be strongly enhanced in this scenario). New multi-bandpass near-infrared spectroscopy of this system would be extremely valuable.

\subsubsection{V893 Scorpius}

V893 Sco is an eclipsing SU UMa system. Hamilton et al. (2011) presented a K-band spectrum obtained using ISAAC on the VLT. They derived a spectral type of M6V. The

spectrum has low $\mathrm{S} / \mathrm{N}$, but boxcar smoothing by 21 pixels, and then running the metallicity analysis, leads to a spectral type of $\mathrm{M} 5.2$, and $[\mathrm{Fe} / \mathrm{H}]=-0.82$. There are no templates in the library with such a low metallicity. The closest, Gl $399(\mathrm{M} 5.0,[\mathrm{Fe} / \mathrm{H}]=-0.58)$, is not a very good match for the spectrum of V893 Sco. While there is a significant $\mathrm{CO} / \mathrm{H}_{2} \mathrm{O}$ break in the spectrum of V893 Sco, the absorption from Na I and CO are extremely weak. Hamilton et al. (2011) examined the luminosity of this CV, and found that it had an observed $K$-band magnitude that was 2.5 mags brighter than an M6V at the distance implied by the parallax. The $K$-band spectrum of V893 Sco suffers from substantial contamination. Multi-bandpass data will be needed to confirm the low metallicity of this system.

\subsubsection{VW Hydri}

The $K$-band spectrum from ISAAC on the VLT was presented in Hamilton et al. (2011). VW Hyi is an SU UMa dwarf novae for which Hamilton et al. estimated a secondary star mass of $0.11 \mathrm{M}_{\odot}$, and derived a spectral type of M4. The metallicity analysis run for this spectrum finds a spectral type of $\mathrm{M} 2.9$, and $[\mathrm{Fe} / \mathrm{H}]=-0.32$. The Newton et al. relation gives $[\mathrm{Fe} / \mathrm{H}]=-0.34$. There are a number of IRTF templates with similar 
properties for comparison. We find the best match occurs with HD95735 (M3.0, [Fe/H] = -0.33), though Gl $213(\mathrm{M} 4.6,[\mathrm{Fe} / \mathrm{H}]=-0.31)$ provides a very similar fit. Thus, the low metallicity is confirmed, and the carbon abundance appears to be normal.

\subsubsection{EX Hydrae}

Hamilton et al. (2011) presented $K$-band spectra for EX Hya, which is a short period IP. They derived a spectral type of M5V. The metallicity analysis on the NIRSPEC spectrum gives a spectral type of M2.0, and $[\mathrm{Fe} / \mathrm{H}]=-0.55$. However, M2V templates, such as Gl 806, have bluer continua than EX Hya. The spectral template Gl $273(\mathrm{M} 4.2,[\mathrm{Fe} / \mathrm{H}]=$ -0.13) provides a better match to the continuum, though all of the absorption features in the spectrum of EX Hya are much weaker than in this object. The low metallicity template Gl 299 (M5.0, $[\mathrm{Fe} / \mathrm{H}]=-0.58)$ provides a good match to the strength of the Na I doublet and Ca I triplet absorption features, though its continuum is too red, and the CO features are weaker in EX Hya. Assuming no contamination in the $K$-band, we conclude that the donor in EX Hya has spectral type near M4, a low metallicity, and $[\mathrm{C} / \mathrm{Fe}] \lesssim-0.30$.

Beuermann et al. (2003) obtained a parallax for EX Hya using the FGS on the HST, giving a distance of $64 \mathrm{pc}$. The 2MASS catalog has $(J-H)=0.32,(H-K)=0.08$, and $K=11.69$. These colors are much bluer than a mid-M type star, confirming the conclusion in Hamilton et al. (2011), that there is some contamination of the $K$-band flux. With the astrometric distance, and the 2MASS $K$-band magnitude, $\mathrm{M}_{K}=7.76$. This is $0.3 \mathrm{mag}$ fainter than an M4V. This suggests that the true spectral type of the donor in EX Hya is closer to M5, and contamination is responsible for the resulting continuum. This would imply an even larger carbon deficit. 


\subsubsection{VY Aquarii}

VY Aqr is an SU UMa-type dwarf nova with an orbital period of $1.51 \mathrm{hr}$. Harrison et al. (2009) present the $K$-band spectrum of this source obtained with NIRSPEC. They showed that the continuum of the observed spectrum was most consistent with an M0V. The metallicity analysis gives a similar answer: K9.6V (and $[\mathrm{Fe} / \mathrm{H}]=+0.37$ ). Harrison et al. found that analysis of archival VLT data for VY Aqr suggested a spectral type closer to M6, though the CO features were much too weak for any M dwarf. The problem with such a classification is that subtraction of a hot blackbody contaminating source does not lead to a realistic spectrum. To merely flatten the spectrum so that it superficially resembles an M dwarf requires us to subtract off a hot source that supplies $95 \%$ of the $K$-band flux. The result is a spectrum with ludicrously strong $\mathrm{Na}$ I and $\mathrm{Ca}$ I absorption features: $[\mathrm{Fe} / \mathrm{H}]=$ 13.9! Though the data are noisy, it is clear that VY Aqr does not have CO in emission. In $§ 5$, we will examine synthetic spectra for stars with hydrogen deficits. Those types of models appear to be required to explain unusual objects like VY Aqr.

Thorstensen (2003) derives an astrometric distance of $97_{-12}^{+15}$ pc to VY Aqr. The 2MASS data for VY Aqr are $(J-H)=0.42,(H-K)=0.27$, and $K=14.59$. There are no AAVSO measurements for this period, but using the NeoWISE database, the faintest VY Aqr gets is $W 1=14.4$. This suggests VY Aqr was in quiescence at the time of the 2MASS observations, giving $\mathrm{M}_{K}=9.5$. Assigning $100 \%$ of the $K$-band luminosity to the donor star, implies a spectral type of M6. While this agrees with the spectral type found by Harrison et al. (2009), the $K$-band spectra remain inconsistent with such a classification. 


\subsubsection{V436 Centauri}

The SU UMa system V436 Cen has a period that is nearly identical to VY Aqr. The $K$-band spectrum has been presented by Hamilton et al. (2011). While the $\mathrm{S} / \mathrm{N}$ of those data are not high, the secondary is quite prominent in the VLT spectrum. Hamilton et

al. estimated a spectral type of M8V for this object. Our analysis derives a spectral type of $\mathrm{M} 6.9$, and $[\mathrm{Fe} / \mathrm{H}]=-0.31$. Fortunately, the IRTF spectral library has several late-type templates for comparison. LP944-20 is listed as having a spectral type of M9, but the metallicity program gives $\mathrm{M} 7.7$, and $[\mathrm{Fe} / \mathrm{H}]=-0.60$. The same analysis for $\mathrm{Gl} 644 \mathrm{c}$ gives M7.4, $[\mathrm{Fe} / \mathrm{H}]=-0.32$. The match of the latter to V436 Cen is much better than the former, and suggests that the metallicity of the donor in V436 Cen is low, while the C abundance is normal.

\subsubsection{V2051 Ophiuchi}

The orbital period of V2051 Oph, an eclipsing SU UMa system, is $6.5 \mathrm{~s}$ shorter than V436 Cen. Hamilton et al. derive a spectral type of M7 for the donor from VLT data. The metallicity analysis estimates a spectral type of $\mathrm{M} 9.2$, and $[\mathrm{Fe} / \mathrm{H}]=-0.53$. There are no templates in the library with this late of spectral type, and this low of an abundance, to use for comparison. Analysis for $\mathrm{Gl} 752 \mathrm{~B}$ gives $\mathrm{M} 8.6$, and $[\mathrm{Fe} / \mathrm{H}]=-0.11$. Gl $752 \mathrm{~B}$ provides an excellent match to the $\mathrm{Na}$ I doublet, and to the $\mathrm{CO}$ features. We conclude that the noisiness of the continuum near $\mathrm{Na} I$ is leading to both a very low value for the metallicity, and the very late spectral type. Except for VY Aqr, all of the donor stars for the SU UMa CVs with periods near $1.5 \mathrm{hr}$ appear to have subsolar metallicities. 


\subsubsection{WX Ceti}

WX Cet is an ultra-short period CV that appears to be an intermediary between the normal SU UMa DNe, and the WZ Sge stars. Sterken et al. (2007) show that WX Cet has normal, short duration SU UMa type eruptions, but also rare superoutbursts of large amplitude. They find that the time between short eruptions is about $\sim 200 \mathrm{~d}$, while superoutbursts occur every $\sim 880 \mathrm{~d}$. These recurrence times are four to five times longer than seen in the typical SU UMa system. WX Cet is extremely faint in the near-IR. It does not appear in the 2MASS Point Source Catalog, though it is present in the $J$-band image. Aperture photometry gives $J_{2 \text { MASS }}=17.2$. The spectrum presented in Fig. 27, has been

smoothed by $30 \AA$. Besides the strong H I and He I emission lines, absorption from the first overtone of ${ }^{12} \mathrm{CO}$ is clearly present. The metallicity regimen assigns a spectral type of M4.9, with $[\mathrm{Fe} / \mathrm{H}]=-0.57$. It is likely that the continuum is contaminated by white dwarf + accretion disk emission. If $25 \%$ of the $K$-band flux is supplied by this source, the estimated spectral type is $\mathrm{M} 6.5$, and the metallicity is $[\mathrm{Fe} / \mathrm{H}]=-0.25$. If the donor provides only $50 \%$ of the $K$-band luminosity, the spectral type is $\mathrm{M} 9.4$, and $[\mathrm{Fe} / \mathrm{H}]=0.35$. The IRTF template Gl $752(\mathrm{M} 8.6,[\mathrm{Fe} / \mathrm{H}]=-0.11)$ is a reasonable match to the spectrum where the secondary supplies $75 \%$ of the $K$-band flux. We conclude that the donor in WX Cet is a late M-type star, and has a near-solar abundance with normal levels of carbon.

\section{Other Interesting Objects}

There are two objects for which we have obtained spectra for that appear to be extremely unusual CVs: QZ Ser and EI Psc. Both appear to have K-type secondary stars, while having short orbital periods. Here we analyze the spectra of these two objects. 


\subsection{QZ Serpentis}

Thorstensen et al. (2002a) recount the discovery of QZ Ser, and used time series spectroscopy to derive an orbital period of $1.996 \mathrm{hr}$. Unexpectedly, for this orbital period, the secondary appeared to have a spectral type of $\mathrm{K} 4 \pm 2$. They also believed that the sodium abundance was probably enhanced. We observed QZ Ser with TripleSpec, and the data are presented in Fig. 28. As found by Thorstensen et al., the continuum of this spectrum is matched by that of a $\mathrm{K} 5 \mathrm{~V}$. Beyond that, the spectrum is highly unusual: the

only emission lines are from He I, there is no evidence for $\mathrm{CO}$ absorption, and the $\mathrm{Na}$ I lines are extremely strong across all bandpasses. Especially prominent is the $\mathrm{Na}$ I doublet at $1.638 \mu \mathrm{m}$, a feature that is usually lost amongst numerous absorption features found in this spectral region. We generated synthetic spectra over a temperature range of $4000 \leq \mathrm{T}_{\text {eff }} \leq$ $5000 \mathrm{~K}, \log g=4.5$, and $-1.00 \leq[\mathrm{Fe} / \mathrm{H}] \leq 0.5$. None of these models matched the depth of the $\mathrm{Na}$ I, Ca I, or the Mg I lines. To fit these common absorption features required huge overabundances of these three elements. After adjusting their abundances, the resulting models fit the strong lines better, but they did not reproduce the depth of the Fe I lines, even in the metal-rich models.

As an alternative, taking the suggestion of Thorstensen et al. that the donor star might be deficient in hydrogen and have enhanced helium abundances, we ran models with solar abundance patterns, while changing the abundances of $\mathrm{H}$ and $\mathrm{He}$. We found the best fitting spectrum occurred when the hydrogen abundance was reduced to $30 \%$ of its normal value, and the helium abundance was increased by a factor of ten. With these changes, iron, magnesium and calcium have normal abundances. This is not true for carbon, sodium, or aluminum. The blue end of the $H$-band is sensitive to both the $\mathrm{H}$ and $\mathrm{C}$ abundances: reduce either one too much, and the continuum becomes too blue. The final model, plotted in Fig. 28, has $[\mathrm{C} / \mathrm{Fe}]=-1.7$ ! The sodium abundance needed to explain the absorption 
features is $32 \times$ the solar value. The aluminum abundance also has to be higher to fit the data, and we found $[\mathrm{Al} / \mathrm{Fe}]=+0.5$. None of our models fit the Na I doublet at $1.638 \mu \mathrm{m}$. This doublet comes from transitions between the $2 \mathrm{p}^{6} 4 \mathrm{p}^{2} \mathrm{P}^{\mathrm{o}}$ and $2 \mathrm{p}^{6} 6 \mathrm{~s}^{2} \mathrm{~S}_{1 / 2}$ (we used the oscillator strengths listed in Cunha et al. 2015). These lines are more prominent in red giants (ibid.), than in dwarfs, and may suggest a lower gravity for this peculiar star.

Obviously, we are in uncharted territory for abundance analysis. To enable us to produce realistic synthetic spectra for an object where the absolute abundances of $\mathrm{H}$ or $\mathrm{He}$ are non-solar, we need to start with a stellar atmosphere that has the correct abundance patterns. While we believe the best solution to the difficulty in modeling the spectrum of QZ Ser is a hydrogen deficiency, in all of our models, the $\mathrm{Na}$ overabundance is very large.

How could such an anomaly arise? There are two proton capture cycles beyond the CNO cycle that are likely responsible for the peculiar abundances of QZ Ser: the NeNa cycle, and the MgAl cycle (see Wallerstein et al. 1997, and references therein). Prantzos et al. (1991) model the production of sodium in 2 and $15 \mathrm{M}_{\odot}$ stars. They found that just before He ignition occurs, the sodium abundance in the innermost regions of the star are enhanced by a factor of five in both models. For the lower mass star, the altered values of $\mathrm{Na}$ were constrained to the inner $0.43 \mathrm{M}_{\odot}$ of the H-exhausted core. Inside this radius, the carbon abundance would also be very low. If the donor star of QZ Ser was once a red giant whose outer atmosphere has been stripped, simple stellar evolution models can probably explain the peculiar Na abundances. This would require an initial mass for the donor in QZ Ser that is higher than typically envisioned under the standard evolutionary paradigm for CVs.

A similar story holds for the $\mathrm{MgAl}$ cycle. Proton capture onto $\mathrm{Mg}$ can result in $\mathrm{Al}$. To operate this cycle, however, appears to require more massive stars $\left(\sim 5 \mathrm{M}_{\odot}\right.$, Ventura et al. 2013). Smiljanic et al. (2016) have surveyed the Na and Al abundances in giants and 
dwarfs. They only found large $\mathrm{Al}$ enhancements for red giants with $\mathrm{M}>3 \mathrm{M}_{\odot}$. It appears that it will require an even more massive progenitor to explain the overabundance of $\mathrm{Al}$ seen in QZ Ser, if it must arise from simple stellar evolution.

Alternatively, the peculiar atmosphere of QZ Ser might be acquired during the common envelope phase of a classical novae $(\mathrm{CNe})$ eruption. The rapid proton capture process (the "rp-process"), during explosive hydrogen burning can produce a wide variety of nucleosynthetic products. Denissenkov et al. (2014) have shown that significant production of $\mathrm{Na}$ or $\mathrm{Al}$ in a $\mathrm{CNe}$ eruption is possible, but almost certainly requires enrichment of material from an underlying ONe white dwarf. These same models also produce significant amounts of carbon. If the relative abundances of our model are even approximately correct, the accretion of CNe processed material cannot explain QZ Ser.

Above, we encountered two systems, VY Aqr and TW Vir, that had anomalously strong metal absorption lines for their spectral types. Instead of having super-solar metallicities, it is just as likely that they suffer from hydrogen deficits. Even an object such as U Gem has some parallels with QZ Ser. Unfortunately, hydrogen-deficient model atmospheres are not yet available for stars cooler than $\mathrm{T}_{\text {eff }} \sim 10,000 \mathrm{~K}$ (cf., Behara \& Jeffery 2006), so further investigation of these CVs is not currently possible.

\subsection{EI Piscium}

Thorstensen et al. (2002b) present spectra and time series photometry of EI Psc and find an ultra-short period of 64 minutes, well below the period minimum for CVs with donor stars of normal composition (see Howell et al. 2001, and references therein). Thorstensen et al. believe that the He abundance is enhanced. Harrison et al. (2009) presented a $K$-band spectrum for EI Psc, obtained with NIRSPEC. As discussed in Harrison et al., and 
Thorstensen et al. (2002b), the spectral type appears to be close to a K5V. The issue with this spectral classification, however, is that the Ca I triplet is much weaker in EI Psc than the Na I doublet (see Fig. 29). For K dwarfs, these two features have a similar strength, and it is not until the mid-M dwarfs $(\sim \mathrm{M} 5 \mathrm{~V})$ where the ratio of the equivalent widths for the Na I and Ca I features becomes as large as seen here (2.2). Like QZ Ser, there is no evidence for $\mathrm{CO}$ absorption features in this spectrum. Unlike QZ Ser, there is $\mathrm{H} \mathrm{I}$ emission. If we assume the secondary has a temperature of $4500 \mathrm{~K}$, the best fit model to the spectrum, has $[\mathrm{Fe} / \mathrm{H}]=-0.2$, and $[\mathrm{C} / \mathrm{Fe}]=-1.7$. Like QZ Ser, Na I appears to be enhanced, and we find $[\mathrm{Na} / \mathrm{Fe}]=+0.9$ provides a good match to the two doublets in the $K$-band.

While we did not need to invoke an $\mathrm{H}$ deficit to explain the NIRSPEC data, it is likely that the short orbital period of this system requires it to be hydrogen deficient. Thorstensen et al. show that if the donor had an initial mass of $1.2 \mathrm{M}_{\odot}$, and mass transfer began shortly after the end of $\mathrm{H}$ burning, the secondary star could evolve to short periods while maintaining a high temperature. This model predicts a depletion of $\mathrm{C}$, and enhanced levels of $\mathrm{N}$ in the photosphere. Using our results for QZ Ser as a guide, if the donor in EI Psc

has an $\mathrm{H}$ deficiency of $30 \%$ like the Thorstensen et al. model, the Na I enhancement would be much smaller, and the abundances of the other metals would have to be substantially subsolar.

\section{Results}

We have used near-IR spectra to derive metallicities and effective temperatures for forty one cataclysmic variables spanning most of the major subclasses. We have extended the program described in $\mathrm{H} \& \mathrm{H}$ to enable us to generate synthetic $I J H K$ spectra, allowing us to model cross-dispersed spectroscopy of CVs we have obtained with SPEX on the 
IRTF, or TripleSpec at APO. We then constructed grids of models to compare to both cross-dispersed and $K$-band spectra for analysis of those CVs with K type secondary stars. We have used the recent metallicity calibrations that employ $K$-band spectra to derive temperatures and values of $[\mathrm{Fe} / \mathrm{H}]$ for $\mathrm{CVs}$ with M-type donor stars. These calibrations were applied to template spectra enabling the direct comparison of similar objects, bolstering the determinations of $\mathrm{T}_{\text {eff }},[\mathrm{Fe} / \mathrm{H}]$, and allowing for estimates of $[\mathrm{C} / \mathrm{Fe}]$. As found in $\mathrm{H} \& \mathrm{H}$, and implied in the data from Harrison et al. (2004a, 2005a,b, 2009), carbon deficits were found for numerous $(21 / 36)$ objects. The trend, however, is that these deficits were more likely to occur in long period $\mathrm{CVs}\left(\mathrm{P}_{\text {orb }}>4 \mathrm{hr}\right)$, then in short period systems. We discuss this, and the other main results in the subsections that follow.

\subsection{Carbon Deficits and CO Emission}

$\mathrm{H} \& \mathrm{H}$ investigated whether it was possible for $\mathrm{CO}$ emission to create apparent carbon deficits. They found that the presence of CO emission, presumably from the accretion disk, overlaying the absorption features of the secondary star creates a spectrum with unique CO absorption feature profiles: blue emission wings, and dramatically narrower bandheads. Using those model results, it is very simple to identify CO emission even in noisy data. We found four CVs with such emission: UU Aql, LS Peg, CN Ori, and CZ Ori. With the inclusion of WZ Sge, this brings the number of CVs that exhibit CO emission to five. None of the other CVs discussed above show evidence for CO emission. Thus, any derived carbon deficits are real, though the quality of the spectra often limited our ability to derive precise values of $[\mathrm{C} / \mathrm{Fe}]$. The ability to compare templates with known metallicities to CVs with M-type secondaries makes the identification of carbon deficits in the shorter period CVs more robust than previous efforts that lacked this information.

One relevant question is why do these five CVs show CO emission? As described in 
$\mathrm{H} \& \mathrm{H}, \mathrm{CO}$ emission is believed to originate in the outer, cooler parts of the accretion disk. We believe that there are three possible explanations. The first is that $\mathrm{CO}$ emission is a transient phenomenon. The second is that the mass transfer rate in these five systems is just right to create the environment amenable to $\mathrm{CO}$ emission. Or third, the structure of their accretion disks is somehow altered by composition, leading to conditions that fosters $\mathrm{CO}$ emission.

Only a larger spectroscopic survey of CVs can answer the first possibility, though the CO emission in WZ Sge was present for at least a full year (see Harrison 2016). The low S/N, low resolution $K$-band spectrum of WZ Sge presented by Dhillon et al. (2000), is highly suggestive of CO emission being present in WZ Sge as far back as 1997. This would rule it out as an artifact of the 2001 eruption. Given its long inter-outburst intervals and low accretion rate, one would expect the disk structure in WZ Sge to be relatively stable. Thus its $\mathrm{CO}$ emission might be persistent. We have no second epoch observations for the other sources with $\mathrm{CO}$ emission. It is interesting to note that the observations of $\mathrm{CN}$ Ori and UU Aql were obtained just a few days after they had returned to minimum light following outbursts.

We have a handful of other objects that have observed more than once. In $\mathrm{H} \& \mathrm{H}$, GK Per, SS Cyg, and RU Peg were observed twice in one week with no change in the CO features. RU Peg and SS Cyg were also previously observed by Harrison et al. (2004a), and Dhillon et al. (2002) observed SS Cyg. There is no evidence for CO emission in those data. We have three epochs of observation for SS Aur, including Harrison et al. (2005a), and it has never shown the signature of $\mathrm{CO}$ emission. It will take additional epochs of observation to ascertain whether $\mathrm{CO}$ emission is transitory.

If we assume $\mathrm{CO}$ emission is tied to accretion rate, the second explanation does not seem especially viable, as LS Peg clearly has a very large accretion rate, while WZ Sge has 
one of the lowest. The third explanation might be a possibility, given that the donors in $\mathrm{CZ}$ Ori, UU Aql and CN Ori all have large subsolar values of $[\mathrm{Fe} / \mathrm{H}]$. Wehrse \& Shaviv (1995) explore the role that metal opacities have on the structure of accretion disks.

The group of objects with the most extreme values for their carbon deficits $([\mathrm{C} / \mathrm{Fe}] \leq$ -1.0) are a heterogeneous set: AE Aqr, CH UMa, V1309 Ori, U Gem, QZ Ser, VY Aqr, and EI Psc. Essentially one object from each of the subclasses in the survey. AE Aqr, U Gem, V1309 Ori and CH UMa were previously shown to have deficits of carbon from UV studies. Hamilton et al. (2011) have discussed the direct correlation between weak CO features in $K$-band spectra, and UV-derived carbon deficits. It is tempting to suggest that like QZ Ser, the objects with the most extreme carbon deficits are hydrogen deficient. In contrast to Harrison et al. (2005b), we now have evidence that polars are not always "normal," as both V1309 Ori and AM Her have carbon deficits.

\subsection{Effective Temperature vs. Orbital Period}

Knigge et al. (2011) published a compilation of the distribution of CV donor spectral types vs. $\mathrm{P}_{\text {orb }}$. They show that a reasonable fit to the distribution results from both standard evolutionary models, as well as models with different angular momentum loss prescriptions. Though the distribution of spectral types in their Fig. 15 could reasonably be summarized by saying that the spectral type of a CV donor star is $\mathrm{M} 4 \pm 2$ for $\mathrm{P}_{\text {orb }}$ $<6 \mathrm{hr}$. The origin of the spectral types plotted in their figure come from a diverse set of methodologies (see Knigge 2006 for references). Given that the majority of the donor stars in the current survey have subsolar metallicities, most of the spectral types in that compilation are, at best, suspect. Some of them have been shown to be wrong (such as the L0 spectral type for VY Aqr). Our version of this diagram (less GK Per) is presented as Fig. 30. We code the various subclasses using different symbols. We use $\mathrm{T}_{\text {eff }}$ in this 
diagram as that is quantity we derive from our synthetic spectra modeling. We use the effective temperature scale from Rajpurohit et al. (2013) to convert M spectral types found above to $\mathrm{T}_{\text {eff }}$.

While there is a general trend of shorter period CVs to have cooler donor stars, the spread at any $\mathrm{P}_{\text {orb }}$ or $\mathrm{T}_{\text {eff }}$ can be significant. For example, there are six CVs with $\mathrm{T}_{\text {eff }}=$ $4750 \mathrm{~K}$ that have orbital periods spanning the range six to ten hours. In contrast, there are ten CVs with $\mathrm{P}_{\text {orb }} \sim 4 \mathrm{hr}$, that span the range $2800 \mathrm{~K} \leq \mathrm{T}_{\text {eff }} \leq 4600 \mathrm{~K}$. A similar grouping is found below $\mathrm{P}_{\text {orb }}<2 \mathrm{hr}$, where $2500 \mathrm{~K} \leq \mathrm{T}_{\text {eff }} \leq 3050 \mathrm{~K}$. Orbital period is a poor predictor for the observed donor star temperature.

Because main sequence stars have a well defined mass-radius relationship, their mean density is therefore defined. Mann et al. (2015) present the relationship for the mass range $0.1 \mathrm{M}_{\odot} \leq \mathrm{M} \leq 0.7 \mathrm{M}_{\odot}$. Fortunately, metallicity plays no significant role. For late-type dwarfs, Mann et al. also found that there is a fairly tight correlation between radius and temperature. Thus, for hydrogen burning main sequence stars, there is an easily defined relationship between mean density and temperature. Given that the mean density of a Roche-lobe filling star is closely tied to orbital period $\left(\langle\rho\rangle=107 \mathrm{P}_{\text {orb }}^{-2} \mathrm{gm} \mathrm{cm}^{-3}\right.$, equation 2.3b in Warner 1995), and the mean density of the Roche lobe filling star is $\langle\rho\rangle=3 \mathrm{M} / 4 \pi \mathrm{R}_{\mathrm{L}}^{3}$, there are only a small number of variables involved that can act to create a range in observed temperature at any particular orbital period. The equivalent spherical radius of the Roche-lobe filling donor star, $\mathrm{R}_{\mathrm{L}}$, is a function of the mass ratio, and dependent on the size of the semi-major axis of the orbit. For $\mathrm{P}_{\text {orb }}=4.3 \mathrm{hr}$ and $\mathrm{M}_{1}=1 \mathrm{M}_{\odot}, \mathrm{R}_{2}=0.5$ and $0.4 \mathrm{R}_{\odot}$, at $q=0.5$ and 0.25 , respectively. For main sequence stars, these two donor stars would differ by $\sim 350 \mathrm{~K}$. Thus, a large difference in the mass ratio can have a significant influence on the observed temperature of the donor star at a fixed orbital period.

Returning to the objects with periods near $4.3 \mathrm{hr}$, the mean density of the donor at 
this period is $6.1 \mathrm{gm} \mathrm{cm}^{-3}$. As shown in Fig. 30, a main sequence star with this density has $\mathrm{T}_{\text {eff }} \sim 3900 \mathrm{~K}$, hotter than that observed for any of the CV donors at this period. That the secondary stars of CVs are cooler than the equivalent main sequence stars has been discussed repeatedly (e.g., Echevarria 1983, Friend et al. 1990, Beuermann et al. 1998, Baraffe \& Kolb 2000). For U Gem, the secondary has a mass of $0.42 \mathrm{M}_{\odot}$, and a mean density of $13.3 \mathrm{gm} \mathrm{cm}^{-3}$. A main sequence star of this mass and density should have a temperature of $3500 \mathrm{~K}(\sim \mathrm{M} 2 \mathrm{~V})$. Yet the best fitting spectral template suggests $\mathrm{T}_{\text {eff }}=$ $3050 \mathrm{~K}$. As discussed earlier, the HST parallax indicated that the donor in U Gem has the luminosity of an M2V. Thus, it is only the observed spectral type that appears incongruent.

As noted above, the large inclination of $\mathrm{U} \operatorname{Gem}\left(i=69^{\circ}\right)$ means that the observed luminosity is dominated by the equatorial regions of the donor, and the enlargement in this direction from the Roche geometry means a lower gravity, and a cooler appearance. In the case of U Gem, the observed spectral type is two subtypes later than expected. SS Aur, with an orbital period very similar to U Gem, assuming all things being equal, should have approximately the same type donor star. The observed spectral type was found to be identical to an M3. As in the case of U Gem, the HST parallax suggested that the luminosity of SS Aur was equivalent to that of an M2V. The observed spectral type would then be one subtype too late. The RKCat lists the inclination of SS Aur as $i=$ $38^{\circ}$. It appears that inclination is playing a significant role in what we observe (we address contamination issues below).

The other non-magnetic systems near this orbital period are RX And $\left(\mathrm{P}_{\text {orb }}=5.03 \mathrm{hr}\right.$, $\left.\mathrm{T}_{\text {eff }}=3500 \mathrm{~K}=\mathrm{M} 1.5 \mathrm{~V}, i=51^{\circ}\right), \mathrm{TW} \operatorname{Vir}\left(\mathrm{P}_{\mathrm{orb}}=4.38 \mathrm{hr}, \mathrm{T}_{\text {eff }}=3600 \mathrm{~K}=\mathrm{M} 1 \mathrm{~V}, i=43^{\circ}\right)$, $\mathrm{CW}$ Mon $\left(\mathrm{P}_{\text {orb }}=4.24 \mathrm{hr}, \mathrm{T}_{\text {eff }}=3300=\mathrm{M} 2.5 \mathrm{~V}, i=65^{\circ}\right)$, and $\mathrm{WW}$ Cet $\left(\mathrm{P}_{\text {orb }}=4.22 \mathrm{hr}\right.$, $\left.\mathrm{T}_{\mathrm{eff}}=3300=\mathrm{M} 2.5 \mathrm{~V}, i=54^{\circ}\right)$. If we blindly apply the offsets found from U Gem and SS Aur to these other systems, we would predict that RX And should have a secondary with a 
spectral type that is slightly earlier than M0V, TW Vir should have an M0V donor, CW Mon should have an M0.5V, and WW Cet should have an M1V. Secondary stars with these spectral types would have temperatures between $\sim 3600 \mathrm{~K}$ and $3900 \mathrm{~K}$, in good agreement with the predictions from the relationship for main sequence stars. KT Per, which has an orbital period of $3.9 \mathrm{hr}$, was found to have an inferred secondary star spectral type that agrees with the main sequence mass-radius relationship at its orbital period (though it has a very low metallicity). The donor in AM Her was found to have a spectral type of M5, while the parallax suggests the luminosity of an M3.5V. The inclination of AM Her is $i=$ $50^{\circ}$.

If inclination is the source of the later-than-expected spectral types in CVs, than we are postulating that the gravity darkening of the secondary stars is responsible for this result. The gravity darkening can be expressed as $\mathrm{T}_{\text {eff }} \propto g^{\beta}$, where $\beta$ is the gravity darkening exponent. For radiative stars $\beta=0.25$, while for convective stars $\beta=0.08$. This assumes that the stars are in radiative equilibrium. If the donor in $U$ Gem is truly an $\mathrm{M} 2 \mathrm{~V}\left(\mathrm{~T}_{\text {eff }}=3400 \mathrm{~K}\right)$, yet it appears to be an $\mathrm{M} 4 \mathrm{~V}\left(\mathrm{~T}_{\text {eff }}=3050 \mathrm{~K}\right)$, and the derived values for the polar and equatorial gravities noted above are assumed, we derive that $\beta=$ 0.09. A highly unusual limb darkening law does not appear to be necessary to explain the observations. However, the inclination of $\mathrm{U}$ Gem is not $90^{\circ}$, but $69^{\circ}$. This would imply that the actual equatorial temperature is cooler than we have found, and the limb darkening exponent would have to be larger than we have derived. Fortunately, Espinosa Lara \& Rieutord (2012) have theoretically investigated the gravity darkening for the Roche-lobe filling component in semi-contact binaries. While not calculating a model with the exact properties of $\mathrm{U}$ Gem, we can interpolate the values of the gravity darkening exponent from their Fig. 4, giving $\beta \simeq 0.22$. For $\mathrm{U}$ Gem, this would imply an equatorial temperature of $\mathrm{T}_{\text {eff }}=2640 \mathrm{~K}$. 
In a similar context, Shahbaz (2003, see also Shahbaz 1998, and Bitner \& Robinson 2006) have constructed the mean spectrum of the Roche-lobe filling secondary in the black hole X-ray transient system GRO J1655-40 (V1033 Sco) in an attempt to measure its mass ratio. For modeling this system, the Roche surface is broken into 2048 surface elements. For each element the surface gravity is determined, and an effective temperature calculated using the appropriate gravity darkening formulation. The most appropriate NEXTGEN model spectrum is then assigned to that element. The spectra for all of the elements are then summed together to create the effective spectrum. For GRO J1655-40, $i=70^{\circ}$, and the secondary star has an apparent spectral type of mid-F. For their models they used both of the two standard limb darkening coefficients $(\beta=0.08$, and $\beta=0.25)$. For the smaller exponent, the temperature across the stellar surface, assuming $\left\langle\mathrm{T}_{\text {eff }}\right\rangle=6336 \mathrm{~K}(\mathrm{~F} 6.5 \mathrm{~V})$, varied from $6490 \mathrm{~K}(\mathrm{~F} 5.5 \mathrm{~V})$ at the poles, to $5120 \mathrm{~K}(\mathrm{~K} 1 \mathrm{~V})$ at the equator. As expected, for $\beta=0.25$, the temperature range was larger: $3230 \mathrm{~K}(\mathrm{M} 3 \mathrm{~V})$ to $6780 \mathrm{~K}(\mathrm{~F} 3 \mathrm{~V})$. In the end, their best fitting model had $\left\langle\mathrm{T}_{\text {eff }}\right\rangle=6600 \mathrm{~K}$ with $\beta=0.08$.

We conclude that for the shorter period systems, inclination appears to be the main source of the "cooler than expected" spectral types. Any remaining scatter can then be attributed to chemical peculiarities in the individual objects, or the various systems could have dramatically different binary mass ratios. With analogies to QZ Ser, the odd abundance patterns in U Gem and TW Vir suggest hydrogen deficiencies, and thus one would not expect them to exactly mimic the properties of normal main sequence stars.

The other main peculiarity in the $\mathrm{P}_{\text {orb }}-\mathrm{T}_{\text {eff }}$ diagram is the existence of a set of six CVs (AE Aqr, CH UMa, V1309 Ori, V426 Oph, SS Cyg, and AH Her), with $\mathrm{T}_{\text {eff }}=4750$ $\mathrm{K}$, but with a large spread in orbital period. In every case, we find that these objects have significant carbon deficiencies. The three longer period CVs of these six appear to have solar values of $[\mathrm{Fe} / \mathrm{H}]$ (ignoring V1309 Ori where we set $[\mathrm{Fe} / \mathrm{H}]=+0.0$ ). SS Cyg and AH 
Her have subsolar metallicities. The most reasonable explanation for this result is that the donor star underwent nuclear evolution prior to the system formally becoming a CV, and these objects have unusual radii and/or masses. RU Peg is an example of a donor in a long period CV that is more than a magnitude more luminous than it would be if it had the radius of a main sequence star.

As first shown in Podsiadlowski et al. (2003), and re-addressed by Goliasch \& Nelson (2015), the inclusion of evolved donors in CV population studies leads to a considerable spread in $\mathrm{T}_{\text {eff }}$ at orbital periods $>5 \mathrm{hr}$. This effect arises due to some donors being zero age main sequence stars at the time they become semi-contact binaries, while others have undergone significant nuclear processing. Our results, combined with the growing numbers of donors in long period CVs that have unusual physical properties (e.g., Connon Smith et

al. 2005, Thoroughgood et al. 2005, Bitner et al. 2007, Echevarria et al. 2007, Neustroev \& Zharikov 2008, Rodríguez-Gil et al. 2009), confirm the conclusions of Baraffe \& Kolb (2000) that CVs with evolved secondaries dominate the population. Many CV donor stars must have had initial masses that were larger than $1 \mathrm{M}_{\odot}$, and our measurements of magnesium deficiencies for several long period CVs strengthens this argument.

\subsection{Contamination Effects}

In the preceding we have mostly ignored the possibility of contamination influencing the determination of donor star spectral types. Here we wish to briefly explore how a hot source, such as the white dwarf, or hot spot on the accretion disk, might alter the spectral type derivation. Qualitatively, the addition of a hot blackbody source to the spectrum of a late-type star should result in the derivation of an earlier spectral type than reality. This argument can be mostly blunted by noting that the depth of various atomic and molecular absorption features are dependent on the temperature of the underlying donor star. Thus, 
the match of the continua and absorption features of two objects with similar metallicities confirms they have similar spectral types.

Here, however, we have the possibility of non-solar metallicities as well as non-standard abundance patterns, and thus cannot blindly assume that a match of the continuum and absorption features in two objects signals concordance of temperature. To test this, we simply have added blackbodies with various fractions of the $K$-band flux to the spectrum of a M-type template star (note that for the analysis of CVs with K-type secondaries, we have continuum divided them, removing any subtle contamination effects). If the contamination is at a low level, $\sim 25 \%$, we find that a donor with a true spectral type of M5V could be confused with an M3V. Larger levels of contamination, however, will not result in further confusion, as the depression of the continuum due to water vapor at both ends of the $K$-band spectrum of an M5V remains imprinted on the continuum of the contaminated spectrum. Thus, the spectrum of a highly contaminated M5V cannot be confused with templates that have spectral a type of $\mathrm{M} 2 \mathrm{~V}$ or earlier.

It is possible for those objects with very low values of $[\mathrm{Fe} / \mathrm{H}]$ to have weak water vapor features. Their continua would be easier to reproduce with larger contamination fractions. The weakening/dilution of the spectral features that comes with a larger contamination would imply an even lower metallicity. This suggests that several of the shortest period systems with the lowest metallicities in Table 3, such EX Hya and V893 Sco, are affected by this process. But we have already identified that these two objects suffer significant contamination. Thus, the process of deriving $\mathrm{T}_{\mathrm{eff}}$ from NIR spectra is surprisingly robust.

The other argument that we are not finding earlier spectral types than reality comes from analysis of those objects with astrometric distances. In nearly every case, the luminosity of the CV system in the $K$-band implies an earlier spectral type for the donor than found here. There is no doubt that the $K$-band spectra of U Gem and SS Aur suffer 
little contamination: Harrison et al. (2000) found that the contamination of the $K$-band luminosities of both objects was less than $5 \%$. Yet we find that in both cases, the observed spectral type is too late to explain their observed $K$-band luminosities. In SS Aur, we need an $\mathrm{M} 2 \mathrm{~V}$ to explain the $K$-band luminosity, while we derive a donor spectral type of $\mathrm{M} 3 \mathrm{~V}$. An M2V is a full magnitude more luminous than an M3V, and the contaminating source would have to supply more than $70 \%$ of the observed flux. In U Gem the situation is even worse, with the contaminating source having to supply over $90 \%$ of the observed luminosity. While contamination is present at some level in every $\mathrm{CV}$, it certainly is not seriously affecting our results.

\subsection{The Metallicities of the Donors in Magnetic CVs}

One of the most consistent findings from our study is the sub-solar metallicities of the IPs and polars. The only real outlier is MQ Dra, which appears to have a slightly super-solar value for $[\mathrm{Fe} / \mathrm{H}]$. MQ Dra (= SDSS1553+5516) has been classified as a low-accretion rate polar (LARP, Schwope et al. 2002). From X-ray observations and the width of the cyclotron features seen in optical spectroscopy, the derived plasma temperatures at the accretion shock region in LARPs are much cooler than typically found in polars $(\sim 1 \mathrm{keV}$, vs. $\gtrsim 5$ $\mathrm{keV})$. The derived accretion rates in LARPs are also very low compared with normal polars $\left(10^{-14} \mathrm{M}_{\odot} \mathrm{yr}^{-1}\right.$, vs. $\left.>10^{-13} \mathrm{M}_{\odot} \mathrm{yr}^{-1}\right)$. In addition, no "high states," periods of increased mass transfer rate that frequently occur in polars, have yet been observed for a LARP. Schmidt et al. (2005) suggest that these objects are "pre-polars," in that the orbital period has not yet evolved to short enough values for the secondaries in these systems to contact their Roche lobes. The cyclotron emission then results from mass transfer through a stellar wind from the donor star.

The position of MQ Dra in the $\mathrm{T}_{\text {eff }}$ vs. $\mathrm{P}_{\text {orb }}$ diagram is very unusual, having a donor 
spectral type that is much cooler than the non-magnetic CVs at its orbital period. The derived spectral type is nearly as late as those seen in the shortest period polars. The derived inclination from light curve modeling in Szkody et al. (2008) indicates an inclination near $i=35^{\circ}$. If we assume it is a normal main sequence star, and its spectral type is 1 subtype earlier than observed, it would have a radius of $\mathrm{R}_{2}=0.16 \mathrm{M}_{\odot}$. To fill its Roche lobe, it would need to have a radius that was twice this size. Schmidt et al. (2005) and Schwartz et al. (2001) found similar results for other LARPs.

The other short period polars would be expected to have temperatures near $\mathrm{T}_{\text {eff }}=$ $3100 \mathrm{~K}(\mathrm{M} 4 \mathrm{~V})$, consistent with our results (not accounting for inclination effects). Thus, the suggestion that LARPs are pre-polars appears to be valid. The super-solar metallicity of MQ Dra could also be interpreted as a sign of youth. It would be extremely useful to obtain $K$-band spectra of all of the other LARPs to explore their metallicities, and to confirm/refine the spectral types of their donor stars.

\section{Conclusions}

We have ascertained the metallicity for the donor stars in a large number of CVs using NIR spectroscopy. For CVs with K-type donor stars, temperatures and abundances have been derived through the comparison of synthetic spectra to observations. For CVs with M-type stars, the values for $[\mathrm{Fe} / \mathrm{H}]$ were derived from recently developed relationships for field $\mathrm{M}$ dwarfs that employ $K$-band spectroscopy. We find that the donor stars in CVs generally have subsolar metallicities. This is consistent with results for field stars in the solar neighborhood, where the local stellar population is shown to be a mix of thin and thick disk stars (Nordström, et al. 2004). An analysis of the kinematics of CVs by Ak et al. (2015) derives a similar result. The donors in longer period CVs appear to have more dramatic abundances anomalies than do the short period systems. In agreement with 
predictions from population synthesis modeling. The presence of $\mathrm{Mg}$ deficits in several long period CVs argues that the donor stars in these systems had large initial masses.

A caveat for the determination of $[\mathrm{Fe} / \mathrm{H}]$ exists for CVs with M-type donors, as variations in the $\mathrm{C} / \mathrm{O}$ ratio could lead to the derivation of artificially low values for $[\mathrm{Fe} / \mathrm{H}]$ (Veyette et al. 2016). This concern is especially relevant given that many CVs have carbon deficits. Our comparison of the high S/N spectrum of U Gem to that of GJ 402 showed that the predicted metallicity offset implied by the observed carbon deficit produced a better agreement between the two objects. However, application of a similar offset to the derived $[\mathrm{Fe} / \mathrm{H}]$ value for carbon-deficient YY/DO Dra made it less consistent with the best-fitting template. Due to the extreme carbon deficits seen in some donors, CVs could provide the data to better establish and/or calibrate the effect of the $\mathrm{C} / \mathrm{O}$ ratio on the metallicity estimates for normal field stars. This could be easily accomplished with new multi-bandpass NIR spectroscopy of those CVs in Table 3 with proven carbon deficits.

It is also now abundantly clear that while the donor stars in CVs may superficially resemble their main sequence cousins, many of them have undergone significant nuclear evolution prior to becoming a CV. The inclusion of such objects into recent population studies is a welcome change, and demonstrates that stellar evolution processes alone are able to explain many of our results. It would be extremely useful to have model atmospheres for cool stars that had both hydrogen deficiencies and enhanced levels of helium, so as to enable the generation of more realistic synthetic spectra. New models for the outbursts of dwarf novae that employ non-solar abundance patterns and/or hydrogen deficiencies should also be considered, so as to investigate whether composition has any affect on outburst properties. The same is true for models of classical novae eruptions, as the composition of the accreted material appears to alter the nature of these events (Shen \& Bildsten 2009).

We close by noting that the total mass of the U Gem system is larger than the 
Chandrasekhar limit, and if the mass lost to CNe eruptions is not too great, the system could become a Type Ia supernova. Note that if the donor star in U Gem is even modestly hydrogen deficient, one of the main arguments against single-degenerate CVs as SNIa progenitors is removed: the lack of $\mathrm{H} \mathrm{I}$ emission lines in their post-deflagration spectra. Pakmor et al. (2008) have found that models for the amount of matter stripped from a main sequence donor star in an SNIa explosion is roughly consistent with the limits implied by observations. Hydrogen deficits in CV donors would only aid this result.

TEH was partially supported by a grant from the NSF (AST-1209451). This publication makes use of data products from the Two Micron All Sky Survey, which is a joint project of the University of Massachusetts and the Infrared Processing and Analysis Center/California Institute of Technology, funded by the National Aeronautics and Space Administration and the National Science Foundation. We acknowledge with thanks the variable star observations from the AAVSO International Database contributed by observers worldwide and used in this research. This publication makes use of data products from NEOWISE, which is a project of the Jet Propulsion Laboratory/California Institute of Technology. NEOWISE is funded by the National Aeronautics and Space Administration. The author wishes to acknowledge S. Howell and P. Szkody who aided in obtaining observations of several of the targets included here. 


\section{References}

Ak, T., Bilir, S., Özd[̈]nmez, A., Soydugan, F., Soydugan, E., et al. 2015, ApSS, 357, 72

Baptista, R., Catalán, M. S., \& Costa, L. 2000, MNRAS, 316, 529

Baraffe, I., Homeier, D., Allard, F., \& Chabrier, G. 2015, A\&A, 577, 42

Baraffe, I., \& Kolb, U. 2000, MNRAS, 318, 354

Bean, J., Sneden, C., Hauschildt, P. H., \& Johns-Krull, C. M., 2006, ApJ, 652, 1604

Behara, N. T., \& Jeffery, C. S. 2006, BaltAst, 15, 115

Beuermann, K., Harrison, T. E., McArthur, B. E., Benedict, G. F., \& Gänsicke, B. T. 2003, A\&A, 412, 821

Beuermann, K., Baraffe, I., Kolb, U., \& Wiechhold, M. 1998, A\&A, 339, 518

Bitner, M. A., Robinson, E. L., \& Behr, B. B. 2007, ApJ, 662, 564

Bitner, M. A., \& Robinson, E. L. 2006, AJ, 131, 1712

Campbell, R. K., Harrison, T. E., \& Kafka, S. 2008, ApJ, 684, 409

Cesetti, M., Pizzella, A., Ivanov, V. D., Morelli, L., Corsini, E. M., et al. 2013, A\&A, 549, 129

Connon Smith, R., Mehes, O., Vande Putte, D., \& Hawkins, N. A. 2005, MNRAS, 360, 364

Covey, K. R., Lada, C. J., Roman-Zuniga, C., Meunch, A. A., Forbrich, J., \& Ascenso, J. 2010, ApJ, 722, 971

Cushing, M.C. Rayner, J.T., \& Vacca, W.D., ApJ, 2005, 623, 1115

Cunha, K., Smith, V. V., Johnson, J. A., Bergemann, M., Mészáros, S., et al. 2015, ApJ, 798, L41

Cushing, M. C., Vacca, W. D., \& Rayner, J. T. 2004, PASP, 116, 362

Denissenkov, P. A., Truran, J. W., Pignatari, M., Trappitsch, Ritter, C. et al. MNRAS, 442,2058

Dhillon, V. S., Littlefair, S. P., Marsh, T. R., Sarna, M. J., \& Boakes, E. H. 2002, A\&A, 393,611 
Dhillon, V. S., Littlefair, S. P., Howell, S. B., Ciardi, D. R., et al. 2000, MNRAS, 314, 826

Echevarria, J., Connon Smith, R., Costero, R., Zharikov, S., \& Michel, R. 2008, MNRAS, 387,1564

Echevarria, J., Michel, R., Costero, R., \& Zharikov, S. 2007, A\&A, 462, 1069

Echevarria, J., 1983 RMxAA, 8, 109

Espinosa Lara, F., \& Rieutord, M. 2012, A\&A, 547, 32

Fernandez, J. M., Latham, D. W., Torres, G., Everett, M. E., Mandushev, G., et al. 2009, ApJ, 701, 764

Friend, M. T., Martin, J. S., Smith, R. C., \& Jones, D. H. P. 1990, MNRAS, 246, 637

Gaidos, E., Mann, A. W., Lépine, S., Buccino, A., James, D., et al. 2014, MNRAS, 443. 2561

Gänsicke, B. T., Szkody, P., de Martino, D., Beuermann, K., Long, K. S., et al. (2003), ApJ, 594, 443

Garnavich, P. M., Szkody, P., Robb, R. M., Zurek, D. R., \& Hoard, D. W. 1994, ApJ, 435, 141

Godon, P., Sion, E. M., Barrett, P. E., \& Linnell, A. P. 2009, ApJ, 699, 1229

Goliasch, J., \& Nelson, L. 2015, ApJ, 809, 80

Gonzalez, G., G., \& Wallerstein, G. 2000, AJ, 119, 1839

Hamilton, R. T., Harrison, T. E., Tappert, C., \& Howell, S. B. 2011, ApJ, 728, 16

Harrison, T. E. 2016, ApJ, 816, 4

Harrison, T. E., \& McArthur, B. E. 2016, AAS 227, 239.07

Harrison, T. E., \& Hamilton, R. T. 2015 (H\&H), AJ, 150, 142

Harrison, T. E., \& Campbell, R. K. 2015, ApJSupp, 219, 32

Harrison, T. E., Bornak, J., McArthur, B. E., \& G. F. Benedict 2013, ApJ, 767, 7

Harrison, T. E., Bornak, J., Rupen, M. P., \& Howell, S. B. 2010, ApJ, 710, 325

Harrison, T. E., Bornak, J., Howell, S. B., Mason, E., Szkody, P., et al. 2009, AJ, 137, 4061 
Harrison, T. E., Campbell, R. K., Howell, S. B., Cordova. F. A., \& Schwope, A. D. 2007a, ApJ, 656, 444

Harrison, T. E., Howell, S. B., Szkdoy, P., \& Cordova, F. A. 2007b, AJ, 133, 162

Harrison, T. E., Howell, S. B., Szkody, P., \& Cordova, F. A. 2005a, ApJ, 632, L123

Harrison, T. E., Osborne, H. L., \& Howell, S. B. 2005b, AJ, 129, 2400

Harrison, T. E., Osborne, H. L., \& Howell, S. B. 2004a, AJ, 127, 3493

Harrison, T. E., Johnson, J. J., McArthur, B. E., Benedict, G. F., Szkody, P. 2004b, AJ, 127,460

Harrison, T. E., McNamara, B. J., Szkody, P., \& Gilliland, R. L. 2000, AJ, 120, 2649

Hessman, F. V., 1988, A\&AS, 72, 515

Houdebine, E. R., \& Mullan, D. J. 2015, ApJ, 801, 106

Howell, S. B., Harrison, T. E., Szkody, P., \& Silvestri, N. M. 2010, AJ, 139, 1771

Howell, S. B., Harrison, T. E., \& Szkody, P. 2004, ApJ, 602, L49

Howell, S. B., Nelson, L. A., \& Rappaport, S. 2001, ApJ, 550, 897

Husser, T. -0., Wende-von Berg, S., Dreizler, S., Homeier, D., Reiners, A., et al. 2013, A\&A, 553, 6

Iben, I., \& Livio, M. 1993, PASP, 150, 1373

Kato, T., Uemura, M., Kiyota, S., Tanabe, K., Koizumi, M. et al. 2003, PASJ, 55, 489

Kolb, U., King, A. R., \& Ritter, H. 1998, MNRAS, 298, 29

Knigge, C., Baraffe, I., \& Patterson, J. 2011, ApJSupp, 194, 28

Knigge, C. 2006, MNRAS, 373, 484

Kraft, R. P. 1962, ApJ, 135, 408

Long, K. S., \& Gilliland, R. L. 1999, ApJ, 511, 916

Mann, A. W., Feiden, G. A., Gaidos, E., Boyajian, T., \& von Braun, K. 2015, ApJ, 804, 64

Mann, A. W., Deacon, N. R., Gaidos, E., Ansdell, M., Brewer, J. M., et al. 2014, AJ, 147, 160 
Mann, A. W., Brewer, J. M., Gaidos, E., Lepine, S., \& Hilton, E. J. 2013, AJ, 145, 52

Marks, P. B., \& Sarna, M. J. 1998, MNRAS, 301, 699

Miller-Jones, J. C. A., Sivakoff, G. R., Knigge, C., Körding, E. G., Templeton, M., et al. 2013, Sci, 340, 950

Naylor, T., Allan, A., \& Long, K. S. 2005, MNRAS, 361, 1091

Newton, E. R., Charbonneau, D., Irwin, J., Berta-Thompson, Z. K., Rojas-Ayala, B., et al. 2014, AJ, 147, 20

Önehag, A., Heiter, U., Gustafsson, B., Piskunov, N., Plez, B., \& Reiners, A. 2012, A\&A, 542,33

Neustroev, V. V., \& Zharikov, S. 2008, MNRAS, 386, 1366

Nordstöm, B., Mayor, M., Andersen, J., Holmberg,J., Pont, F., et al. 2004, A\&A, 418, 989

Pakmor, R., Röpke, F. K., Weiss, A., \& Hilllebrandt, W. 2008, A\&A, 489, 943

Parsons, S. G., Marsh, T. R., Copperwheat, C. M., Dhillon, V. S., Littlefair, S. P., et al. 2010, MNRAS, 402, 2591

Patterson, J. 1998, PASP, 110, 1132

Patterson, J., \& Szkody, P. 1993, PASP, 105, 1116

Patterson, J., \& Eisenman, N. 1987, IBVS 3079

Pecaut, M. J., \& Mamajek, E. E. 2013, ApJS, 208, 9

Podsiadlowski, Ph., Han, Z., \& Rappaport, S. 2003, MNRAS, 340, 1214

Politano, M., \& Weiler, K. P. 2007, ApJ, 665, 663

Prantzos, N., Coc, A., \& Thibaud, J. P. 1991, ApJ, 379, 729

Rajpurohit, A. S., Rehlé, Allard, F., Homeier, D., Schultheis, M., et al. 2013, A\&A, 556, 15

Ramsay, G., Wheatley, P. J., Norton, A. J., Hakala, P., \& Baskill, D. 2008, MNRAS, 387, 1157

Ribeiro, T., Baptista, R., Harlaftis, E. T., Dhillon, V. S., \& Rutten, R. G. M. 2007, A\&A, 474, 213 
Ringwald, F. A., Thorstensen, J. R., Honeycutt, R. K., \& Smith, R. C. 1996, AJ, 111, 2077

Ringwald, F. A., Thorstensen, J. R., \& Hamwey, R. M. 1994, MNRAS, 271, 323

Ritter, H., \& Kolb, U. 2003, A\&A, 404, 301

Rodríguez-Gil, P., Torres, M. A. P., Gänsicke, B. T., Muñoz-Darias, T., Steeghs, D., et al. 2009, A\&A, 496, 805

Rojas-Ayala, B., Covey, K. R., Muirhead, P. S., \& Lloyd, J. P. 2010, ApJL, 720, L113

Schmidt, G. D. , Szkody, P., Vanlandingham, K. M., Anderson, S. F., Barentine, J. C., et al. 2005, ApJ, 630, 1037

Schwartz, R., Schwope, A. D., \& Staude, A. 2001, A\&A, 374, 189

Schwope, A. D., Brunner, H., Hambaryan, V., Schwarz, R., Staude, A., et al. 2002, in ASP

Conf. Vol. 261, The Physics of Cataclysmic Variables and Related Objects, ed. B.T.

Gänsicke, K. Beuermann, \& K. Reinsch (San Francisco: ASP), 102

Schreiber, M. R., Gänsicke, B. T., \& Mattei, J. A. 2002, A\&A, 384, 6

Sepinski, J. F., Sion, E. M., Szkody, P., \& Gänsicke, B. T. 2002, ApJ, 574, 937

Shahbaz, T. 2003, MNRAS, 339, 1031

Shahbaz, T. 1998, MNRAS, 298, 153 Shen, K. J., \& Bildsten, L. 2009, ApJ, 692, 324

Sion, E. M., Godon, P., Cheng, F., \& Szkody, P. 2007, AJ, 134, 886

Sion, E. M., Cheng, F. H., Godon, P., \& Szkody, P. 2004, RevMex, 20, 194

Sion, E. M., Winter, L., Urban, J. A., Tovmassian, G. H., Zharikov, S., et al. 2004, AJ, 128,1795

Smiljanic, R., Romano, D., Bragaglia, A., Donati, P., Magrini, L., et al. 2016, A\&A, 589, 115

Smith, V. V., Cunha, K., Shetrone, M. D., Meszaros, S.,Allende Prieto, C., et al. 2013, 765,16

Sneden, C. 1973, PhD thesis, Univ. Texas

Sterken, C., Vogt, N., Schreiber, M. R., Uemura, M., \& Tuvikene, T. 2007, A\&A, 463, 1053 
Szkody, P., Linnell, A. P., Campbell, R. K., Plotkin, R. M., Harrison, T. E., et al. 2008, ApJ, 683, 967

Szkody, P., Nishikida, K., Erb, D., Mukai, K., Hellier, C., et al. 2002, AJ, 123, 413

Szkody, P., \& Silber, A. 1996, AJ, 112, 289

Szkody, P., Williams, R. E., Margon, B., Howell, S. B., \& Mateo, M. 1992, ApJ, 387, 357

Szkody, P., \& Mateo, M. 1986, ApJ, 301, 286

Szkody, P., \& Capps, R. W. 1980, AJ, 85, 882

Taylor, C. J., Thorstensen, J. R., \& Patterson, J. 1999, PASP, 111, 184

Thoroughgood, T. D., Dhillon, V. S., Steeghs, D., Watson, C. A., Buckley, D. A. H., et al. 2005, MNRAS, 357, 881

Thorstensen, J. R., Lepine, S., \& Shara, M. 2008, AJ, 136, 2107

Thorstensen, J. R., Fenton, W. H., \& Taylor, C. J. 2004a, PASP, 116, 300

Thorstensen, J. R. 2003, AJ, 126, 3017

Thorstensen, J. R., Fenton, W. H., Patterson, J., Kemp, J., Halpern, J., et al. 2002a, PASP, 114, 1117

Thorstensen, J. R., Fenton, W. H., Patterson, J. O., Kemp, J., Krajci, T. et al. 2002b, ApJ, 567, L49

Thorstensen, J. R., \& Ringwald, F. A. 1997, PASP, 109, 483

Ventura, P., Di Criscienzo, M., Carini, R., \& D'Antona, F. 2013, MNRAS, 431, 3642

Verbunt, F., Bunk, W. H., Ritter, H., \& Pfeffermann, E. 1997, A\&A, 327, 602

Verbunt, F., \& Zwaan, C. 1981, A\&A, 100, 7

Veyette, M. J., Muirhead, P. S., Mann, A. W., \& Allard, F. 2016, arXiv160504904

von Zeipel, H. 1924, MNRAS, 84, 665

Wallerstein, G., Iben, I., Parker, P., Boesgaard, A. M., Hale, G. M. 1997, RvMP, 69, 995

Warner B., 1995, Cataclysmic Variable Stars. Cambridge Univ. Press, Cambridge, p 33

Watson, C. A., Dhillon, V. S., Shahbaz, T. 2006, MNRAS, 368, 637 
Wehrse, R. \& Shaviv, G. 1995, in ASP Conf. Ser. 78, Astrophysical Applications of

Powerful New Databases, ed. S. J. Adelman \& W.L. Wiese (San Francisco, CA: ASP), p301

Wilson, J. C., Henderson, C. P., Herter, T. L., Matthews, K., Skrutskie, M. F., et al. 2004, SPIE, 5492, 1295

Wing, R. F., \& Jørgensen, U. G. 2003, JAAVSO, 31, 110

Woolf, V. M., \& West, A. W. 2012, MNRAS, 422, 1489 
Table 1. Observation Log

Object UT Date Start/Stop Times Exp. Instrument $\mathrm{P}_{\text {orb }}(\mathrm{hr})$ Obs. Phase State

\begin{tabular}{lccccccc} 
GK Per & $2004-08-15$ & $13: 53$ to $14: 19$ & $240 \mathrm{~s}$ & SPEX & 48.1 & 0.94 & Min. \\
EY Cyg & $2004-08-16$ & $11: 05$ to $11: 57$ & $180 \mathrm{~s}$ & SPEX & 11.02 & 0.97 & Min. \\
AE Aqr & $2004-08-15$ & $08: 05$ to $09: 48$ & $90 \mathrm{~s}$ & SPEX & 9.88 & 0.61 & Min. \\
RU Peg & $2004-08-15$ & $10: 25$ to $10: 59$ & $240 \mathrm{~s}$ & SPEX & 8.99 & 0.85 & Min. \\
SS Cyg & $2004-08-15$ & $08: 55$ to $09: 20$ & $120 \mathrm{~s}$ & SPEX & 6.60 & 0.40 & Min. \\
CZ Ori & $2005-02-17$ & $05: 00$ to $05: 20$ & $180 \mathrm{~s}$ & NIRSPEC & 5.25 & 0.66 & Nearing min. \\
RX And & $2004-08-15$ & $13: 07$ to $13: 41$ & $240 \mathrm{~s}$ & SPEX & 5.03 & 0.59 & Min. \\
SS Aur & $2005-09-01$ & $15: 02$ to $15: 26$ & $240 \mathrm{~s}$ & SPEX & 4.39 & 0.12 & Min. \\
SS Aur & $2007-03-05$ & $06: 00$ to $07: 18$ & $120 \mathrm{~s}$ & NIRSPEC & 4.39 & 0.99 & Min. \\
U Gem & $2012-02-03$ & $02: 22$ to $07: 51$ & $240 \mathrm{~s}$ & TSPEC & 4.25 & $\ldots$ & Min. \\
U Gem & $2012-02-03$ & $08: 40$ to $10: 34$ & $240 \mathrm{~s}$ & TSPEC & 4.25 & $\ldots$ & Min. \\
CW Mon & $2005-02-17$ & $05: 29$ to $05: 40$ & $180 \mathrm{~s}$ & NIRSPEC & 4.24 & 0.28 & Min. \\
WW Cet & $2004-08-15$ & $12: 20$ to $12: 54$ & $240 \mathrm{~s}$ & SPEX & 4.22 & 0.60 & Min. \\
LS Peg & $2004-08-15$ & $11: 34$ to $12: 08$ & $240 \mathrm{~s}$ & SPEX & 4.19 & 0.68 & Min. \\
IP Peg & $2003-09-06$ & $06: 39$ to $07: 00$ & $120 \mathrm{~s}$ & NIRSPEC & 3.98 & 0.03 & Min. \\
DO Dra & $2005-02-17$ & $09: 50$ to $09: 59$ & $240 \mathrm{~s}$ & NIRSPEC & 3.97 & 0.86 & Min. \\
CN Ori & $2005-02-17$ & $05: 44$ to $05: 49$ & $180 \mathrm{~s}$ & NIRSPEC & 3.92 & 0.33 & Min. \\
KT Per & $2004-08-16$ & $14: 31$ to $14: 56$ & $240 \mathrm{~s}$ & SPEX & 3.90 & 0.16 & Intermed. \\
TT Ari & $2004-08-15$ & $14: 56$ to $15: 22$ & $240 \mathrm{~s}$ & SPEX & 3.30 & 0.08 & Min. \\
QZ Ser & $2012-02-03$ & $11: 08$ to $12: 45$ & $240 \mathrm{~s}$ & TSPEC & 2.00 & 0.67 & $V=15.6$ \\
\hline & & & & & &
\end{tabular}




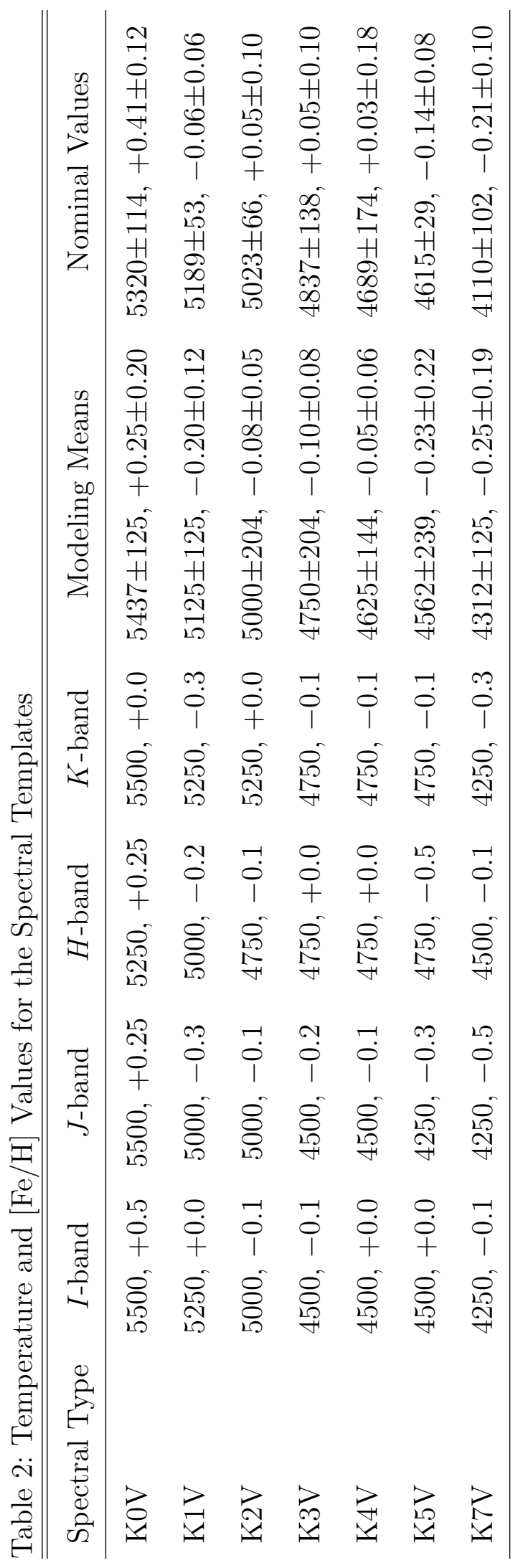


Table 3. Observation Log

\begin{tabular}{|c|c|c|c|c|c|c|}
\hline Object & Subtype & $\mathrm{P}_{\text {orb }}$ & $\mathrm{T}_{\mathrm{eff}}$ & {$[\mathrm{Fe} / \mathrm{H}]$} & {$[\mathrm{C} / \mathrm{Fe}]$} & Other Abundances \\
\hline GK Per & IP & 47.92 & 5000 & -0.3 & -0.5 & {$[\mathrm{Mg} / \mathrm{Fe}]=-0.3$} \\
\hline EY Cyg & $\mathrm{UG}$ & 11.02 & 5250 & +0.0 & -0.5 & $\cdots$ \\
\hline AE Aqr & IP & 9.88 & 4750 & +0.0 & -1.0 & $\cdots$ \\
\hline SY Cnc & $\mathrm{ZC}$ & 9.18 & 5500 & +0.0 & +0.0 & $\cdots$ \\
\hline RU Peg & SS & 8.99 & 5000 & -0.3 & -0.4 & {$[\mathrm{Mg} / \mathrm{Fe}]=-0.14$} \\
\hline CH UMa & $\mathrm{UG}$ & 8.24 & 4750 & +0.0 & -1.0 & $\cdots$ \\
\hline V1309 Ori & $\mathrm{AM}$ & 7.98 & 4750 & $+0.0=$ & -1.0 & {$[\mathrm{Mg} / \mathrm{Fe}]=-0.5$} \\
\hline EM Cyg & $\mathrm{ZC}$ & 6.98 & 4500 & -0.5 & -0.2 & $\cdots$ \\
\hline V426 Oph & $\mathrm{ZC}$ & 6.85 & 4750 & +0.0 & -0.5 & $\cdots$ \\
\hline SS Cyg & $\mathrm{UG}$ & 6.60 & 4750 & -0.3 & -0.4 & {$[\mathrm{Mg} / \mathrm{Fe}]=-0.3$} \\
\hline TT Crt & $\mathrm{UG}$ & 6.44 & 4500 & +0.0 & -0.5 & $\cdots$ \\
\hline AH Her & $\mathrm{UG} / \mathrm{ZC}$ & 6.19 & 4750 & -0.7 & -0.4 & $\cdots$ \\
\hline CZ Ori & $\mathrm{UG}$ & 5.25 & 4250 & -1.0 & $+0.0=$ & $\cdots$ \\
\hline EX Dra & $\mathrm{UG}$ & 5.04 & 4000 & -0.5 & -0.2 & {$[\mathrm{Mg} / \mathrm{Fe}]=+0.0$} \\
\hline RX And & $\mathrm{ZC} ?$ & 5.03 & 3500 & +0.1 & +0.0 & $\cdots$ \\
\hline MQ Dra & $\mathrm{AM}$ & 4.39 & 2800 & +0.2 & +0.0 & $\cdots$ \\
\hline SS Aur & UG & 4.39 & 3100 & -0.1 & +0.0 & $\cdots$ \\
\hline TW Vir & UG & 4.38 & 3600 & +0.6 & $\cdots$ & $\cdots$ \\
\hline U Gem & $\mathrm{UG}$ & 4.25 & 3050 & +0.2 & -1.0 & $\cdots$ \\
\hline CW Mon & $\mathrm{UG}$ & 4.24 & 3300 & -0.2 & +0.0 & $\cdots$ \\
\hline WW Cet & $\mathrm{UG}$ & 4.22 & 3300 & -0.3 & $\geq-0.3$ & $\cdots$ \\
\hline
\end{tabular}


Table 3-Continued

\begin{tabular}{lcccccl}
\hline \hline \multicolumn{1}{c}{ Object } & Subtype & $\mathrm{P}_{\text {orb }}$ & $\mathrm{T}_{\text {eff }}$ & {$[\mathrm{Fe} / \mathrm{H}]$} & {$[\mathrm{C} / \mathrm{Fe}]$} & Other Abundances \\
\hline & & & & & & \\
YY/DO Dra & $\mathrm{IP}$ & 3.97 & 3200 & -0.2 & $\leq-0.3$ & $\ldots$ \\
UU Aql & $\mathrm{UG}$ & 3.92 & 3375 & -0.7 & $\ldots$ & $\ldots$ \\
CN Ori & $\mathrm{UG}$ & 3.92 & 3375 & -0.7 & $\ldots$ & $\ldots$ \\
KT Per & ZC & 3.90 & 3580 & -0.5 & +0.0 & $\ldots$ \\
IP Peg & $\mathrm{UG}$ & 3.80 & 3625 & +0.2 & $\leq 0.0$ & $\ldots$ \\
AM Her & $\mathrm{AM}$ & 3.09 & 2900 & -0.4 & $\leq 0.0$ & $\ldots$ \\
QZ Ser & $\mathrm{SU}$ & 2.00 & 4530 & $+0.0=$ & -1.7 & {$[\mathrm{Na} / \mathrm{Fe}]=+1.5,[\mathrm{Al} / \mathrm{Fe}]=+0.5$} \\
AR UMa & $\mathrm{AM}$ & 1.93 & 2925 & -0.5 & +0.0 & $\ldots$ \\
ST LMi & $\mathrm{AM}$ & 1.90 & 2775 & -0.4 & +0.0 & $\ldots$ \\
MR Ser & $\mathrm{AM}$ & 1.89 & 3000 & -0.2 & +0.0 & $\ldots$ \\
RZ Leo & $\mathrm{WZ}$ & 1.83 & 2925 & -0.3 & $\leq 0.0$ & $\ldots$ \\
V893 Sco & $\mathrm{SU}$ & 1.82 & 2860 & -0.8 & $\ldots$ & $\ldots$ \\
VW Hyi & $\mathrm{SU}$ & 1.78 & 3050 & -0.3 & +0.0 & $\ldots$ \\
VV Pup & $\mathrm{AM}$ & 1.67 & 2690 & -0.3 & +0.0 & $\ldots$ \\
EX Hya & $\mathrm{IP}$ & 1.64 & 3050 & -0.6 & $\leq-0.3$ & $\ldots$ \\
VY Aqr & $\mathrm{SU}$ & 1.51 & 2800 & +0.4 & $\vdots-1.0$ & $\ldots$ \\
V436 Cen & $\mathrm{SU}$ & 1.50 & 2670 & -0.3 & +0.0 & $\ldots$ \\
V2051 Oph & $\mathrm{SU}$ & 1.50 & 2500 & -0.1 & +0.0 & $\ldots$ \\
WX Cet & $\mathrm{SU}$ & 1.40 & 2600 & +0.0 & $\ldots$ & $\ldots$ \\
EI Psc & $\mathrm{SU}$ & 1.07 & 4530 & -0.2 & -1.7 & {$[\mathrm{Na} / \mathrm{Fe}]=+0.9$} \\
\hline & & & & & &
\end{tabular}




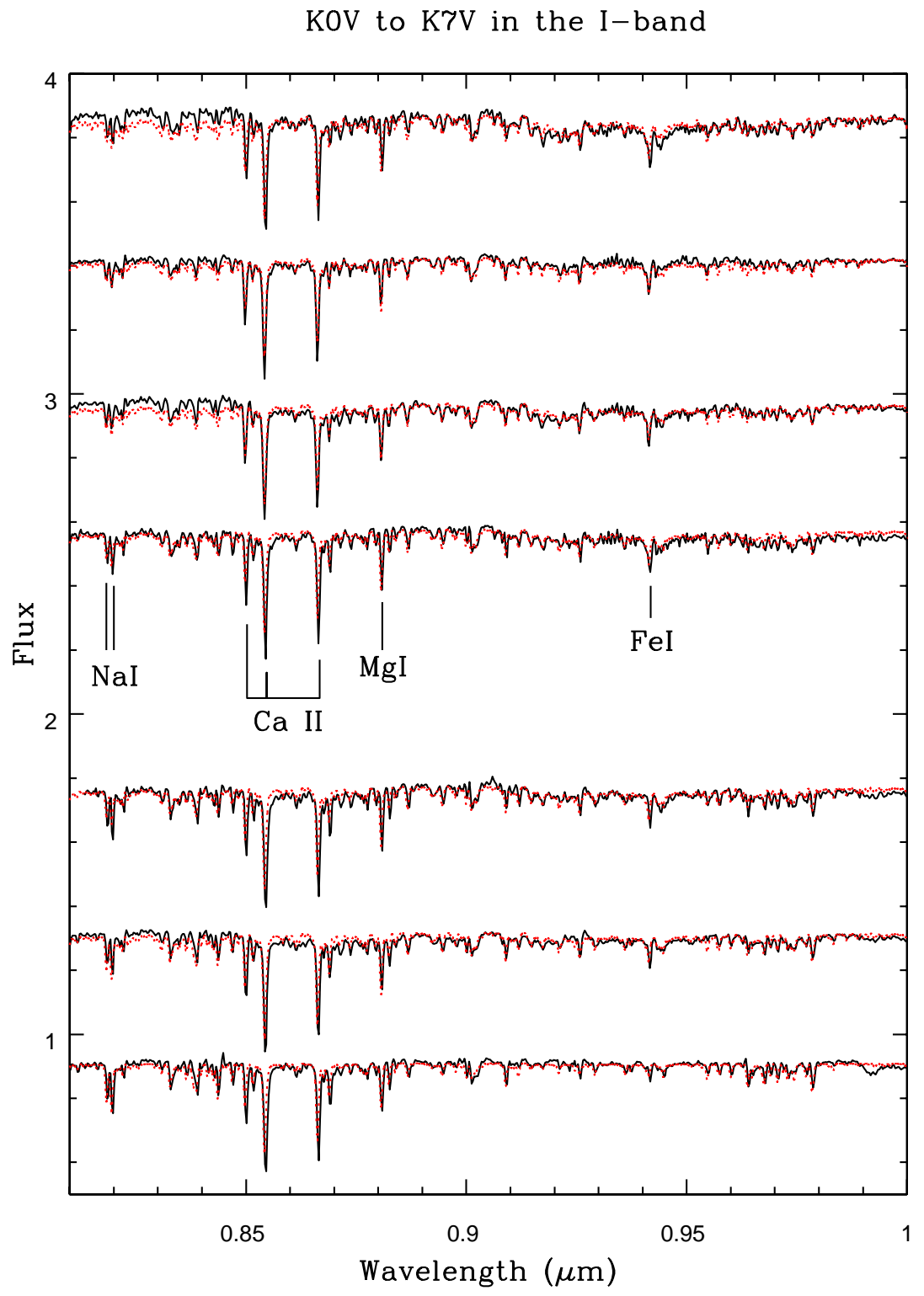

Fig. 1a.- Spectra of the $\mathrm{K}$ dwarf templates, K0V top, K7V bottom (black), over the wavelength range $0.81 \mu \mathrm{m} \leq \lambda \leq 1.0 \mu \mathrm{m}$. The synthetic model spectra generated using SPECTRUM are in red. The strongest absorption lines have been identified. 


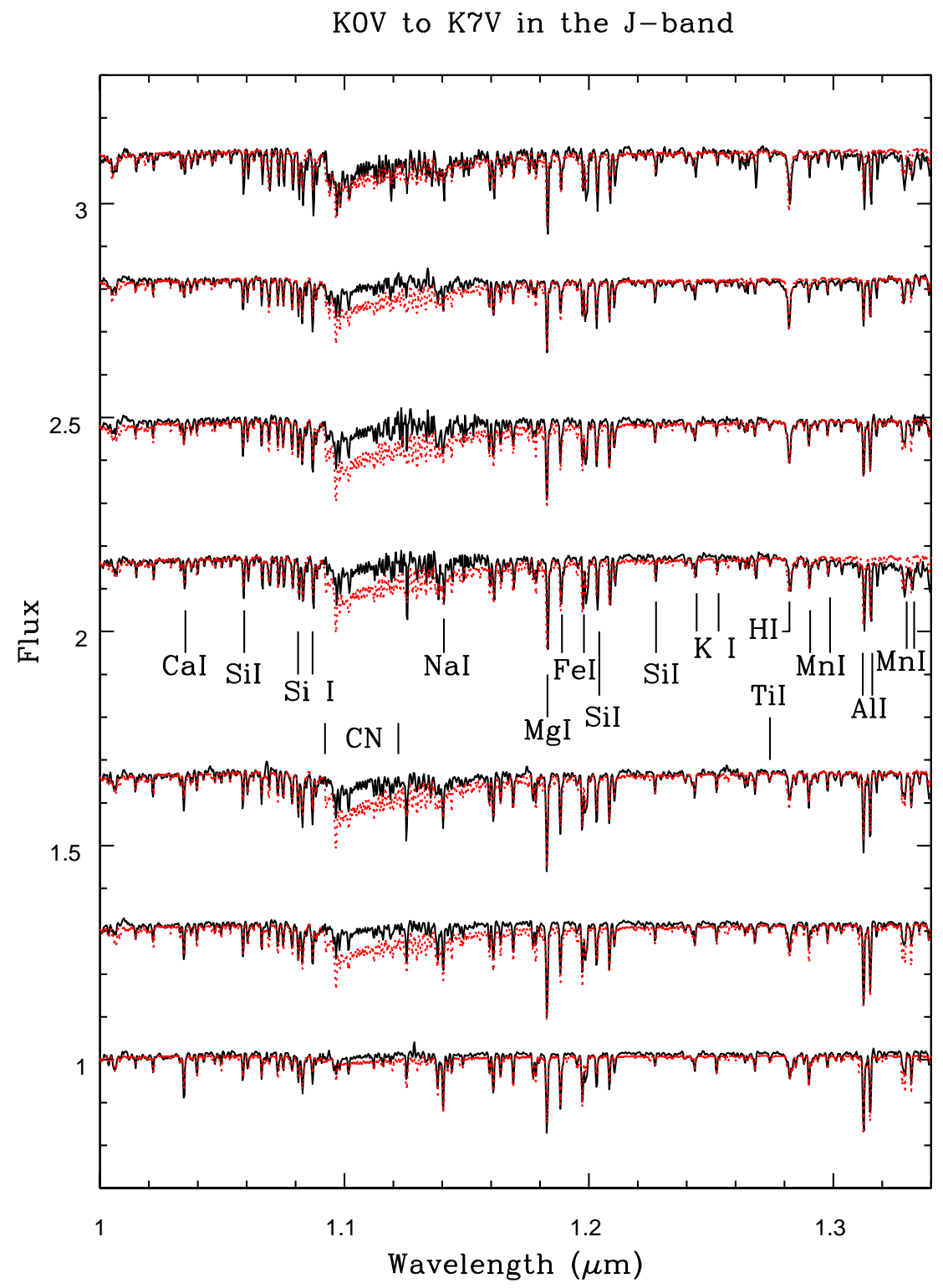

Fig. 1b. - The same as 1a, but for the wavelength interval $1.0 \mu \mathrm{m} \leq \lambda \leq 1.34 \mu \mathrm{m}$. 


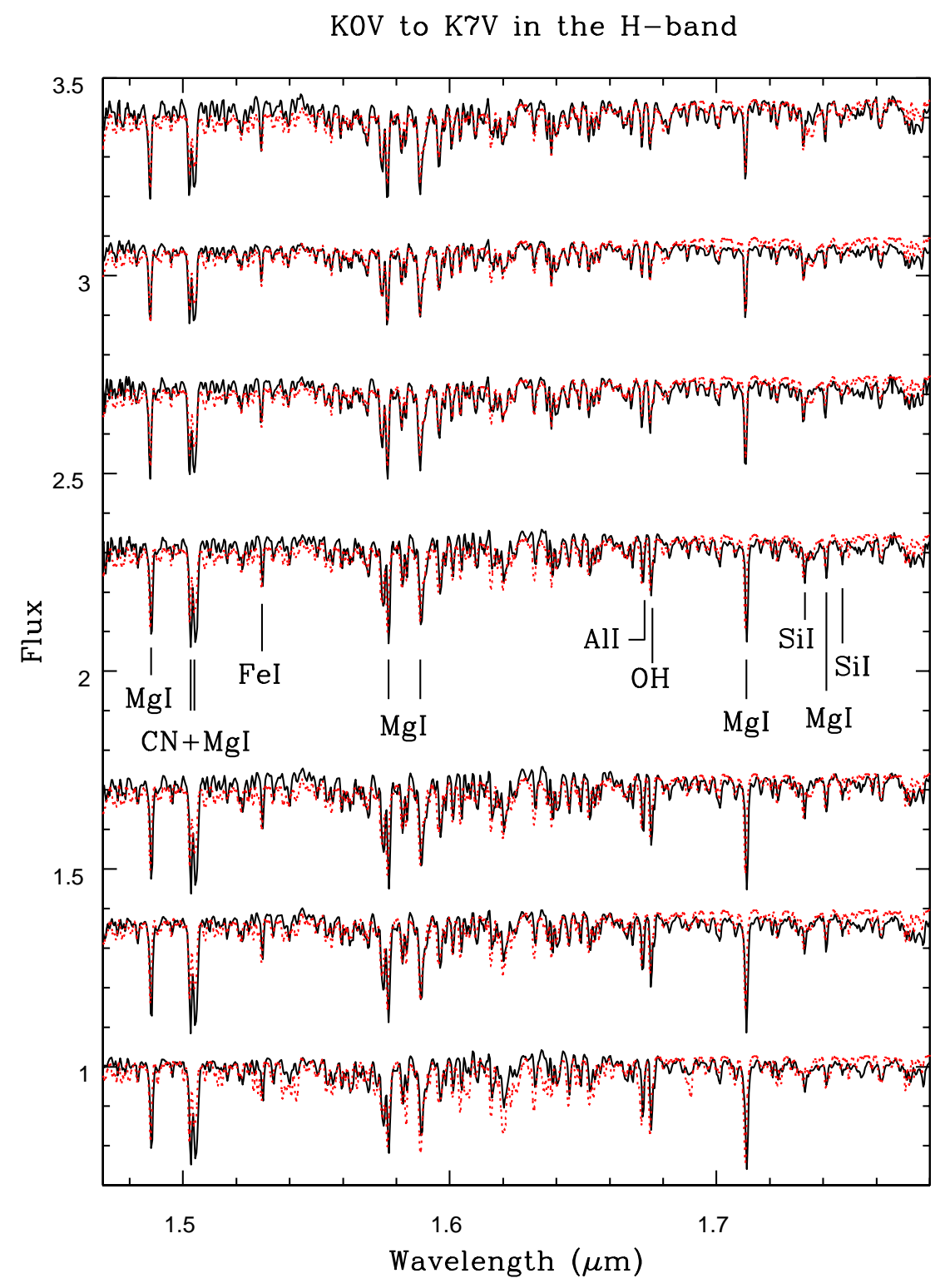

Fig. 1c. - As in 1a, but for the wavelength interval $1.47 \mu \mathrm{m} \leq \lambda \leq 1.78 \mu \mathrm{m}$. 


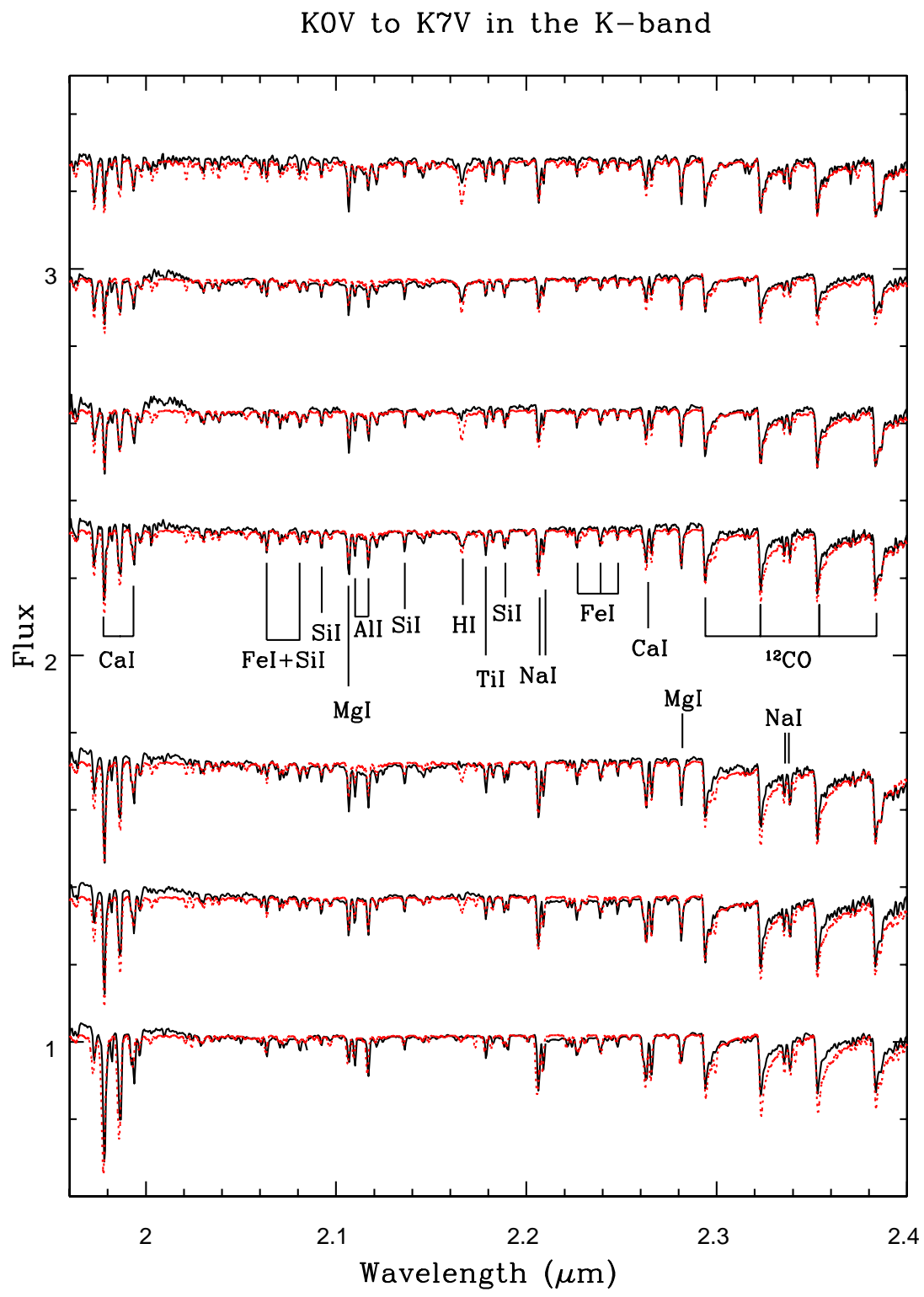

Fig. 1d.- As in 1a, but for the wavelength interval $1.96 \mu \mathrm{m} \leq \lambda \leq 2.4 \mu \mathrm{m}$. 

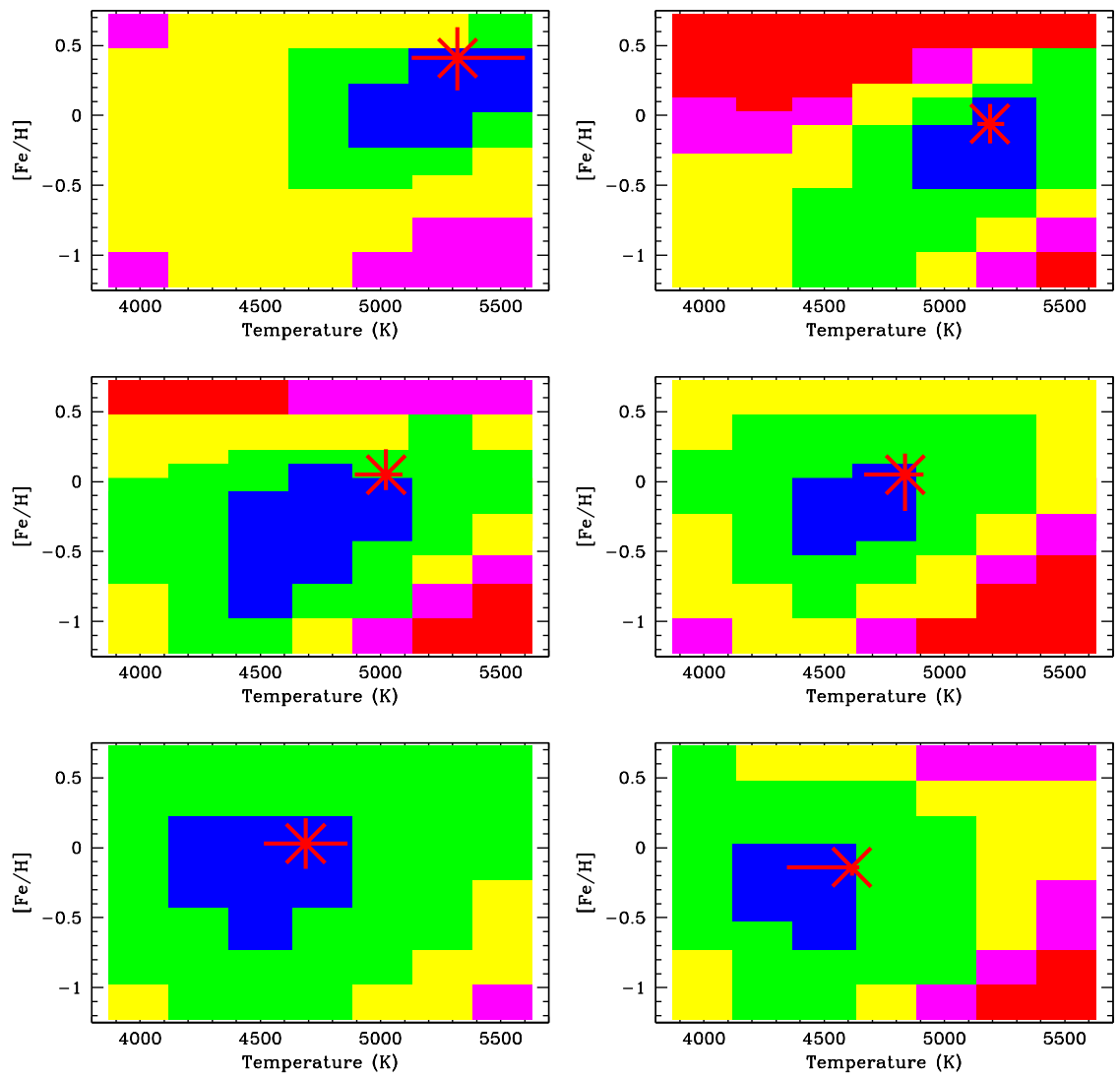

Fig. 2.- The heat maps resulting from a $\chi^{2}$ analysis of a grid of synthetic spectra compared to those of the $\mathrm{K}$ dwarf templates (K0V top left, $\mathrm{K} 5 \mathrm{~V}$ bottom right, $\mathrm{K} 7 \mathrm{~V}$ not shown) for the J-band. The red "X" in each panel marks the location of the nominal value for $\mathrm{T}_{\text {eff }}$ and $[\mathrm{Fe} / \mathrm{H}]$. The line segments that pass through the vertex of the each of the $\mathrm{Xs}$ are not error bars, but display the full range of published measurements for each star (see Table 2 for their formal error bars). In this heat map, blue denotes $\chi_{\text {red }}^{2} \leq 0.7$, green is $0.7 \leq \chi_{\text {red }}^{2}<$ 0.9 , yellow is $0.9 \leq \chi_{\text {red }}^{2}<1.5$, magenta is $1.5 \leq \chi_{\text {red }}^{2}<2.5$, and red is $\chi_{\text {red }}^{2} \geq 2.5$. 

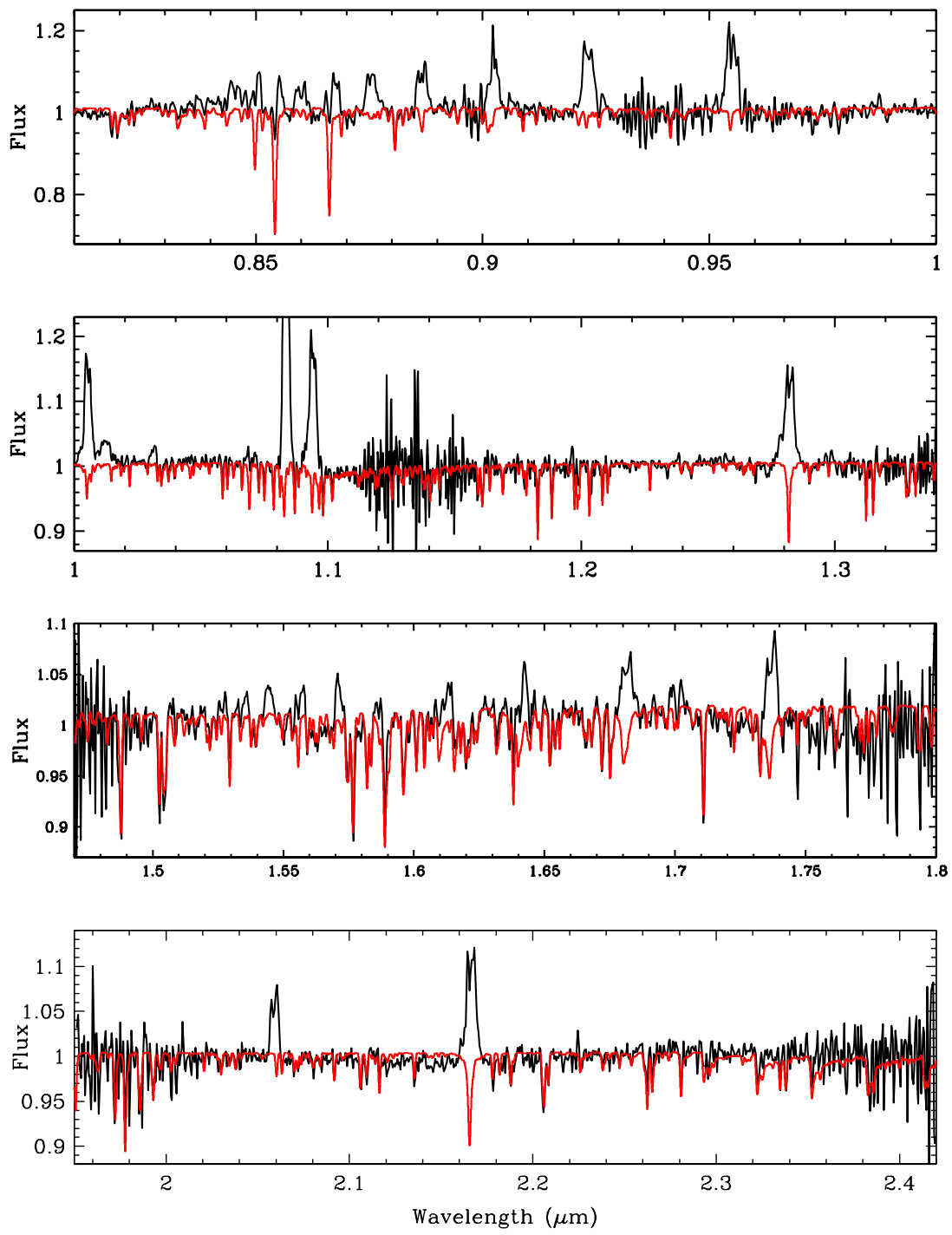

Fig. 3.- The spectrum of GK Per (black) and the best fitting model spectrum (red). 

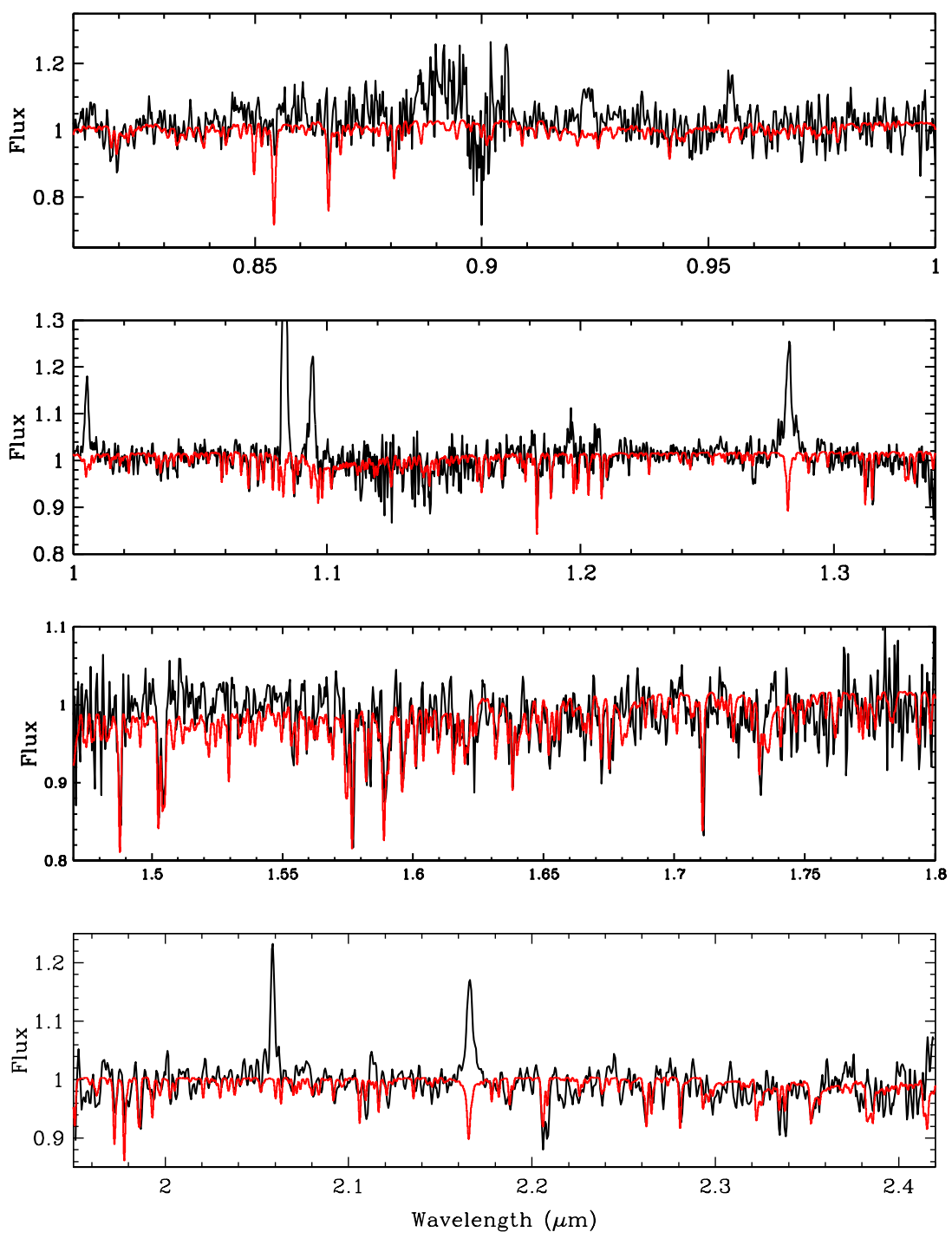

Fig. 4. - The spectrum of EY Cyg (black) and the best fitting model spectrum (red). 

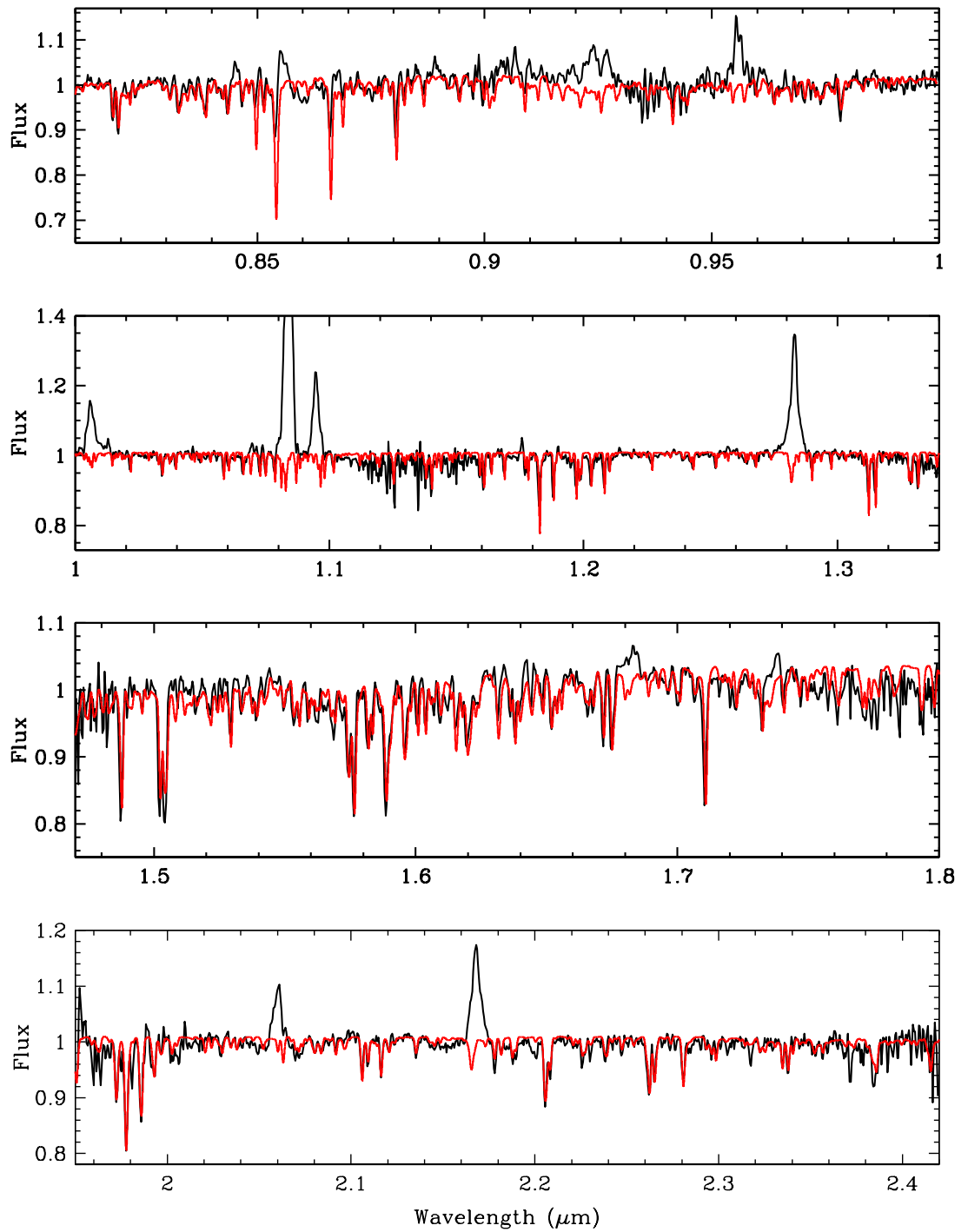

Fig. 5.- The spectrum of AE Aqr (black) and the best fitting model spectrum (red). 

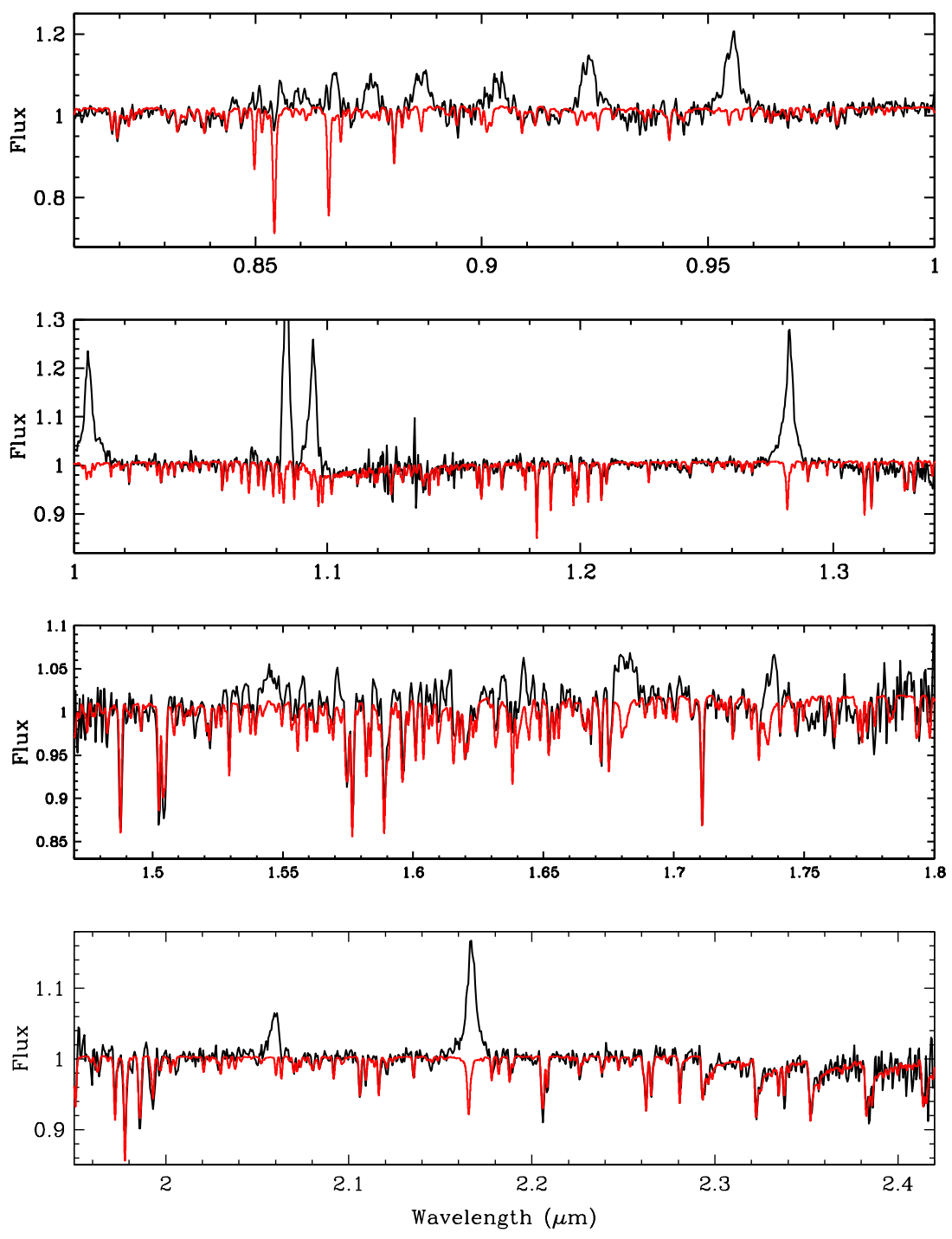

Fig. 6. - The spectrum of RU Peg (black) and the best fitting model spectrum (red). 

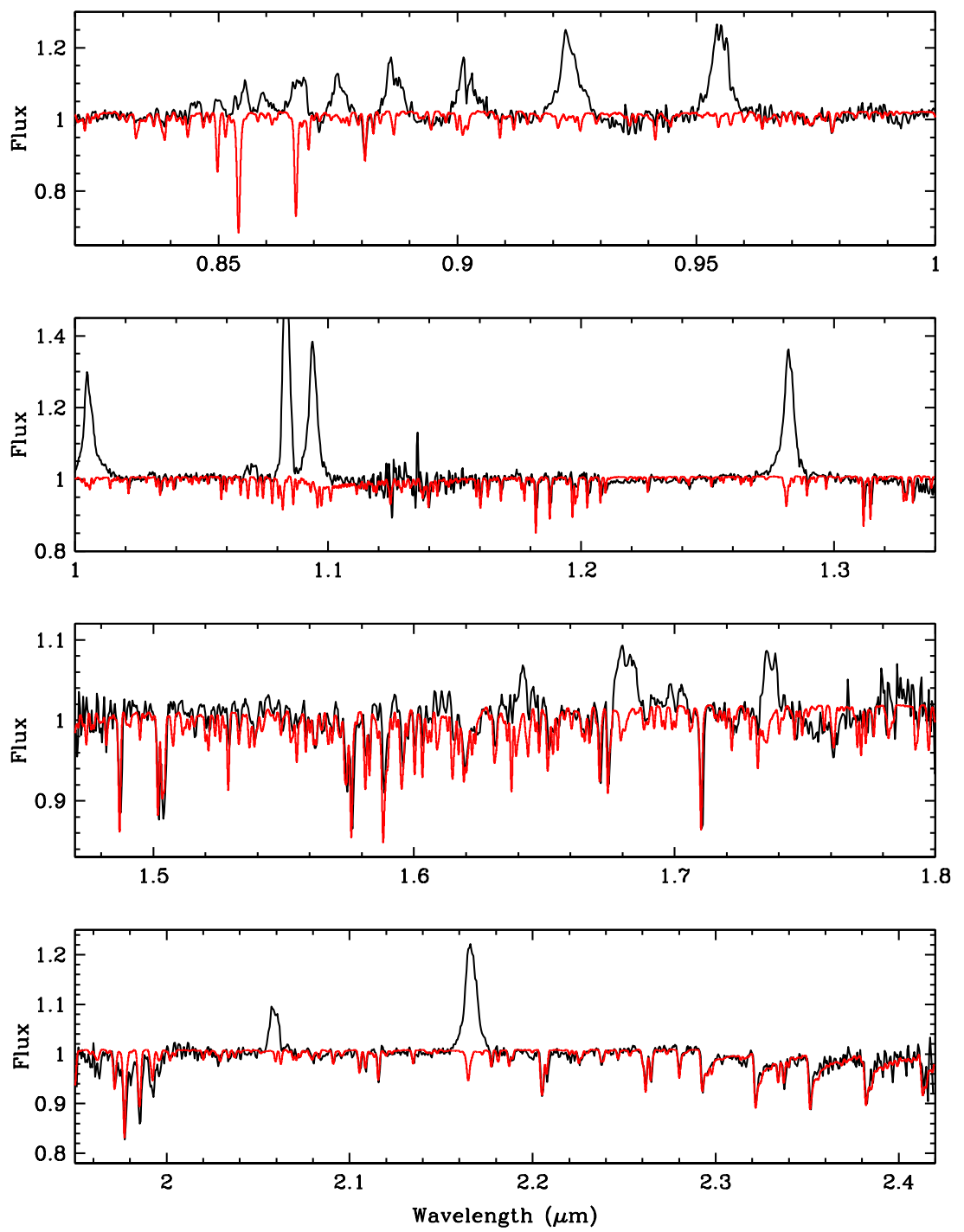

Fig. 7.- The spectrum of SS Cyg (black) and the best fitting model spectrum (red). 

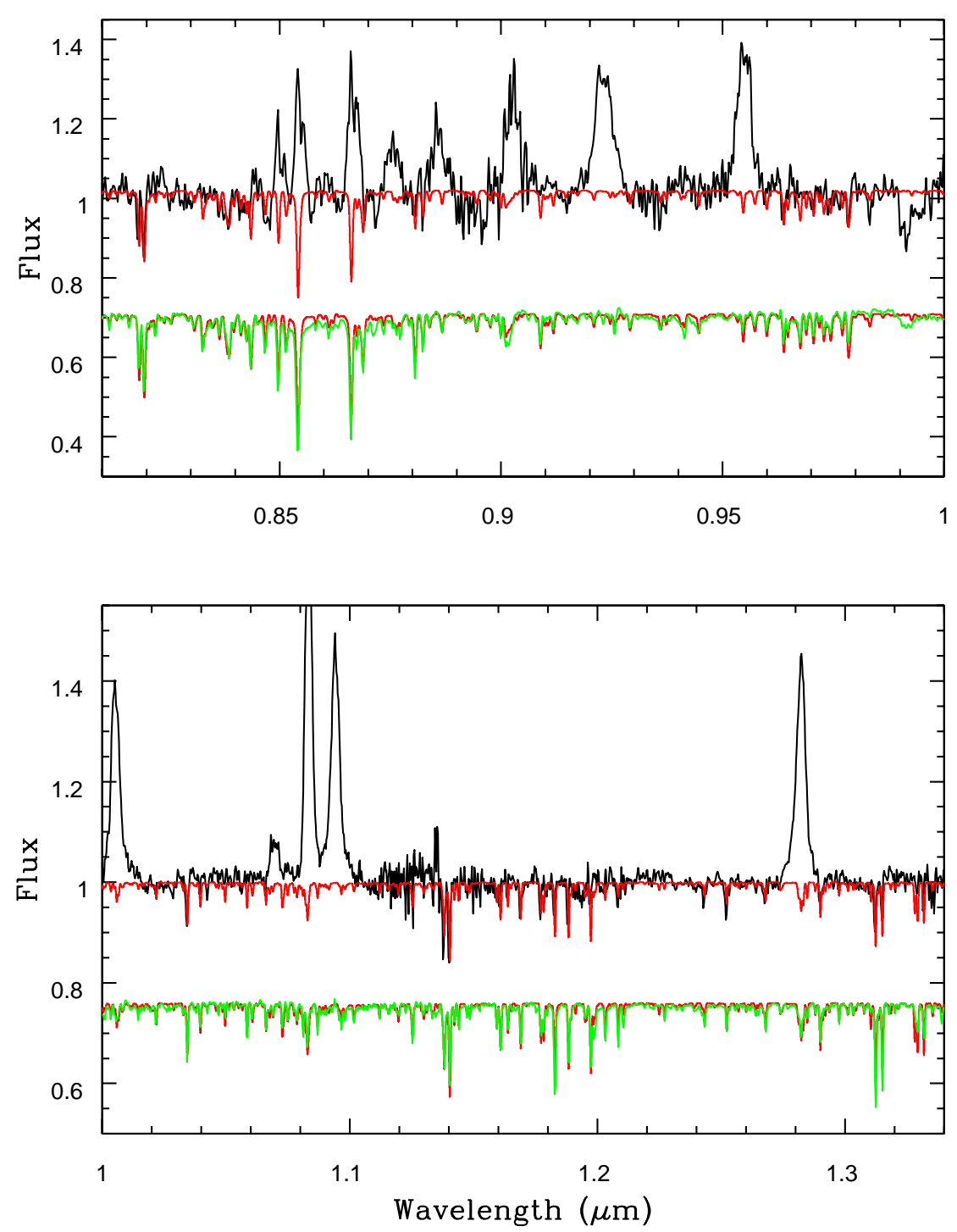

Fig. 8a.- The spectrum of RX And (black) and HD 19305 (green) in the $I$-band (top panel) and in the $J$-band (bottom panel). For HD 19305 a model spectrum with $\mathrm{T}_{\text {eff }}=3750 \mathrm{~K}$ and $[\mathrm{Fe} / \mathrm{H}]=0.0$ is plotted in red. For RX And, the synthetic spectrum also has $\mathrm{T}_{\text {eff }}=3750 \mathrm{~K}$, but $[\mathrm{Fe} / \mathrm{H}]=-0.3$. 

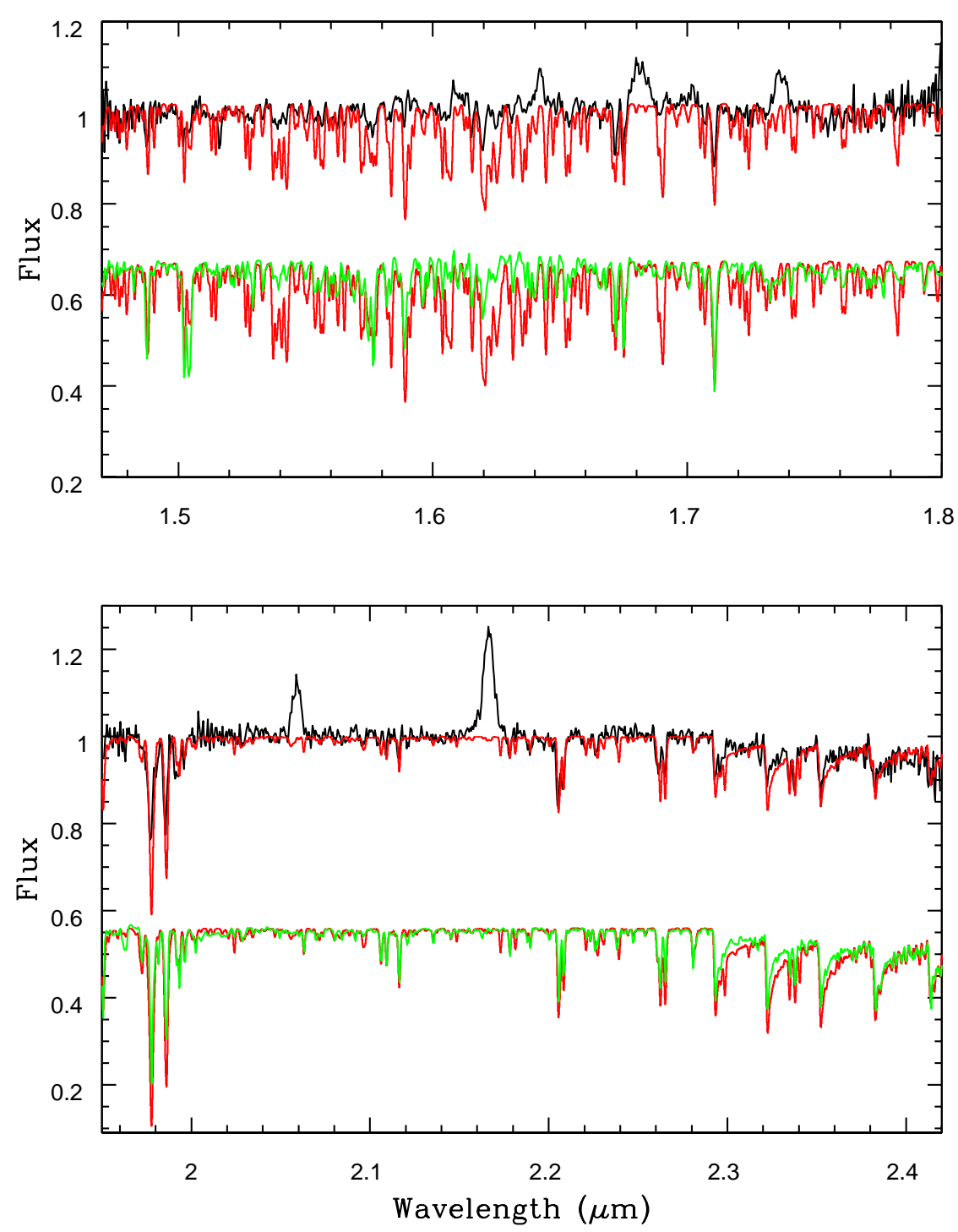

Fig. 8b.- The same as 7a, but for the $H$-band (top), and $K$-band (bottom). 


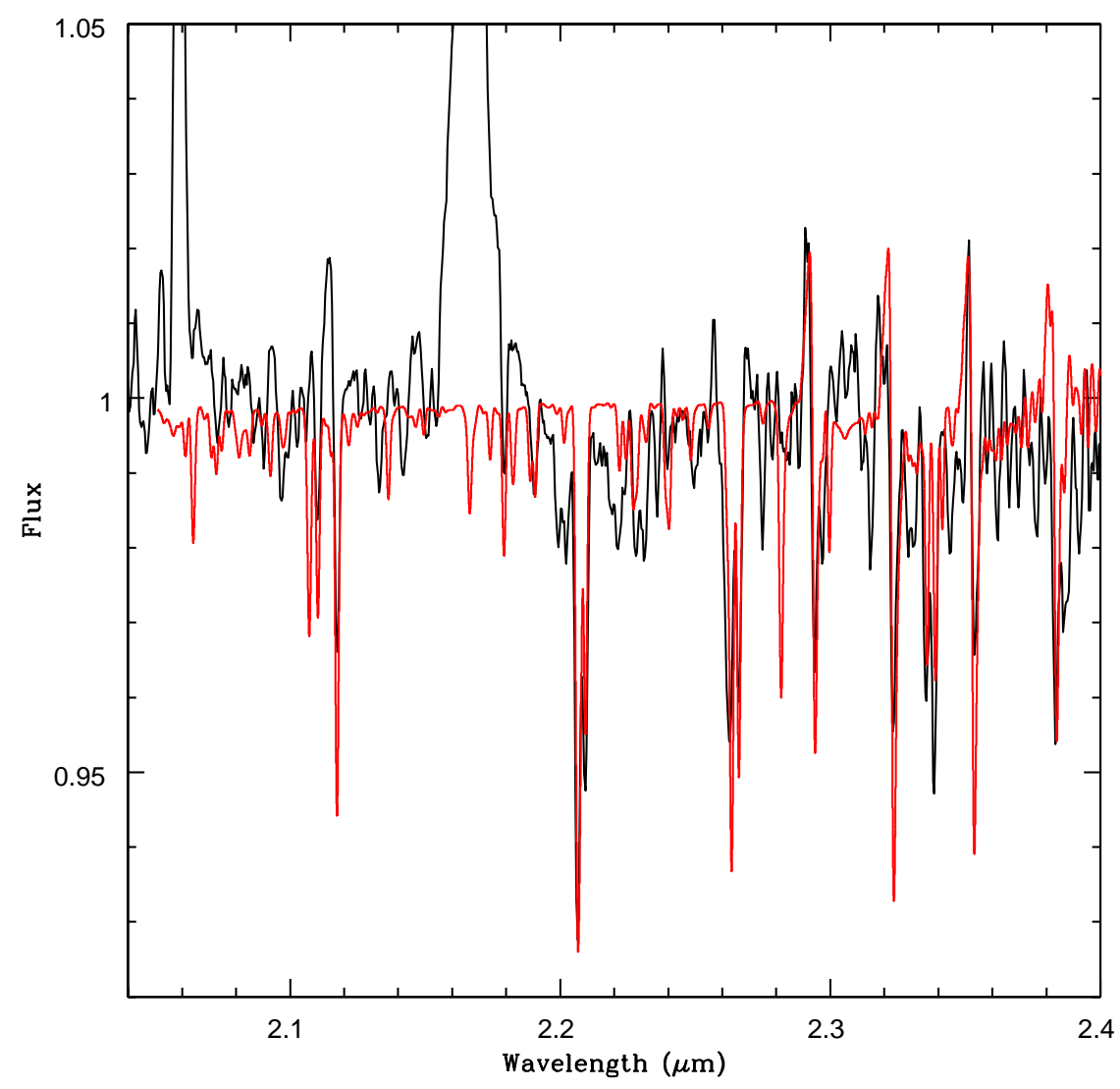

Fig. 9.- The NIRSPEC $K$-band spectrum of CZ Ori (black) with a CO emission spectrum with $\left(v \sin i=800 \mathrm{~km} \mathrm{~s}^{-1}\right)$ that has been added to a synthetic spectrum (red) with $\mathrm{T}_{\text {eff }}=$ $4250 \mathrm{~K}$, and $[\mathrm{Fe} / \mathrm{H}]=-1.0$ 

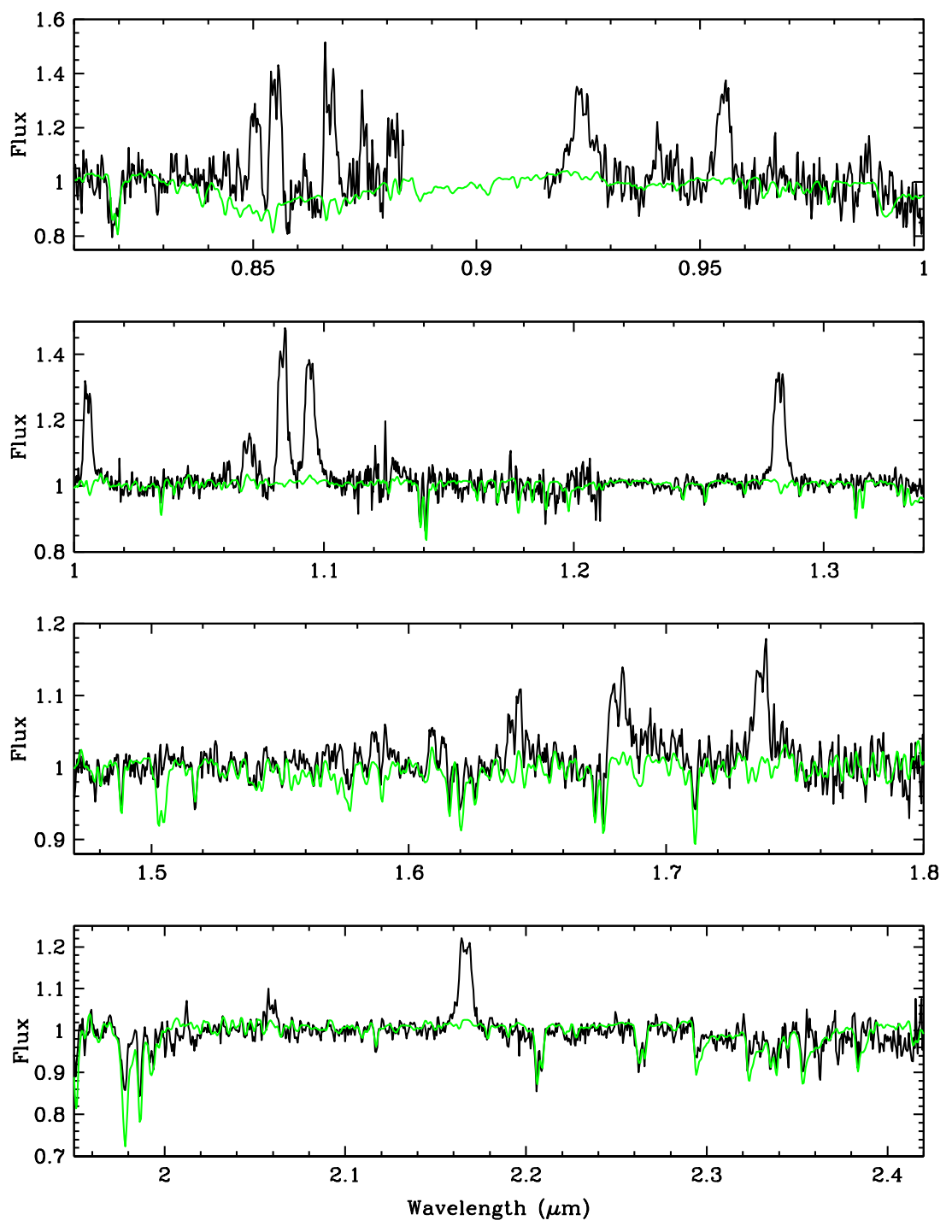

Fig. 10.- The SPEX data for SS Aur (black) compared to Gl 388 (green), an M3V with $[\mathrm{Fe} / \mathrm{H}]=+0.11$. We have rotationally broadened the template spectrum to a velocity of $v \sin i=125 \mathrm{~km} \mathrm{~s}^{-1}$ to better match SS Aur. 


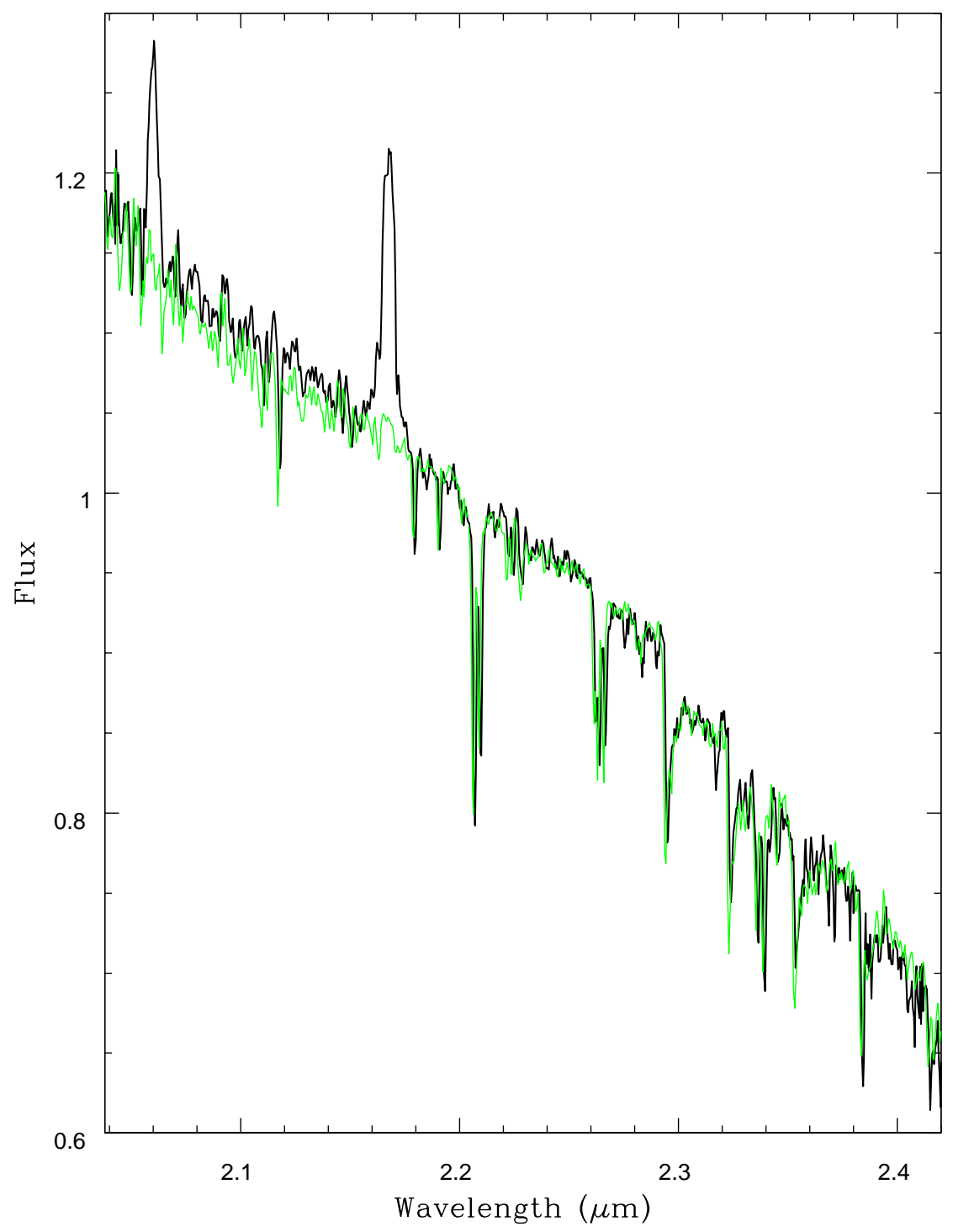

Fig. 11. - The NIRSPEC K-band data for SS Aur (black) compared to Gl 388 (green). 

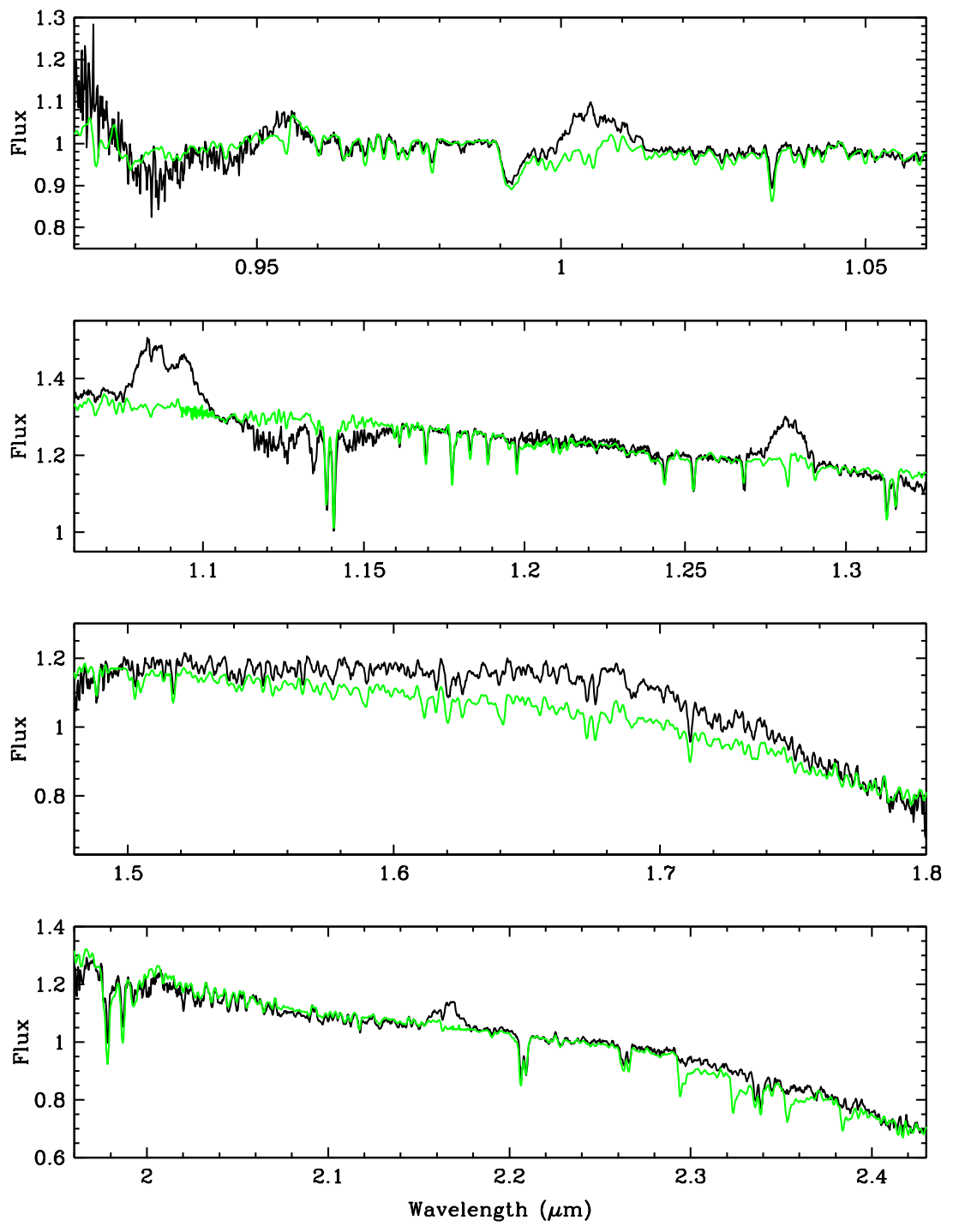

Fig. 12. - The TripleSpec spectra of U Gem (black), and GJ 402 (green). Both objects appear to have super-solar metallicities, however, U Gem has extremely weak CO features. The deviation of the continuum in the $H$-band corresponds with the shape of the $\mathrm{H}^{-}$opacity minimum, suggesting that the secondary star of U Gem has a lower gravity than GJ 402. 

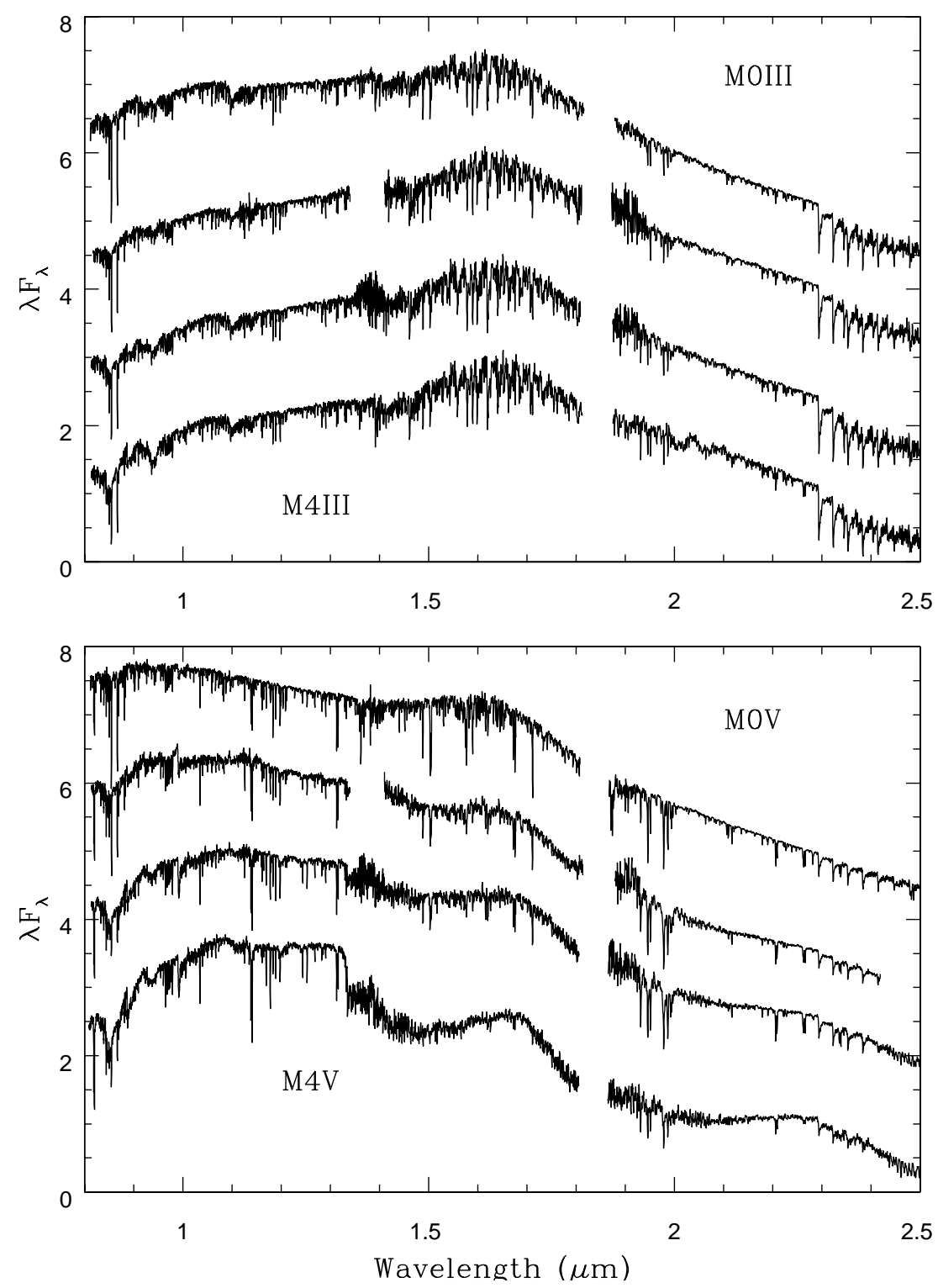

Fig. 13.- The NIR spectra of M giants (top panel) and M dwarfs (bottom panel), from M0 to M4 (from the IRTF Spectral Libary). 


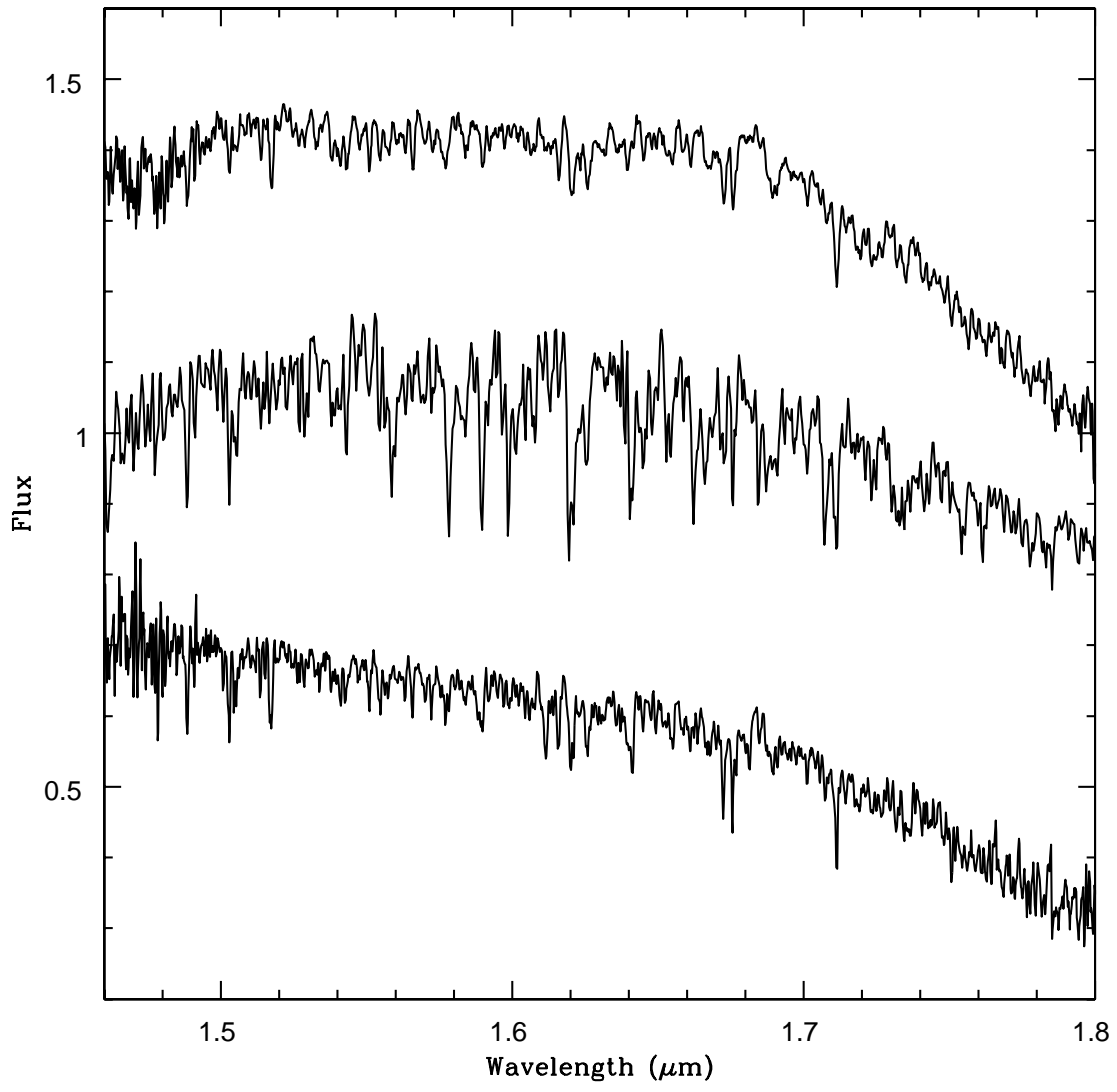

Fig. 14. - The $H$-band spectra of U Gem (top) and GJ 402 (bottom), compared to that of HD4408 (middle), an M4III (from the IRTF Spectral Libary). 


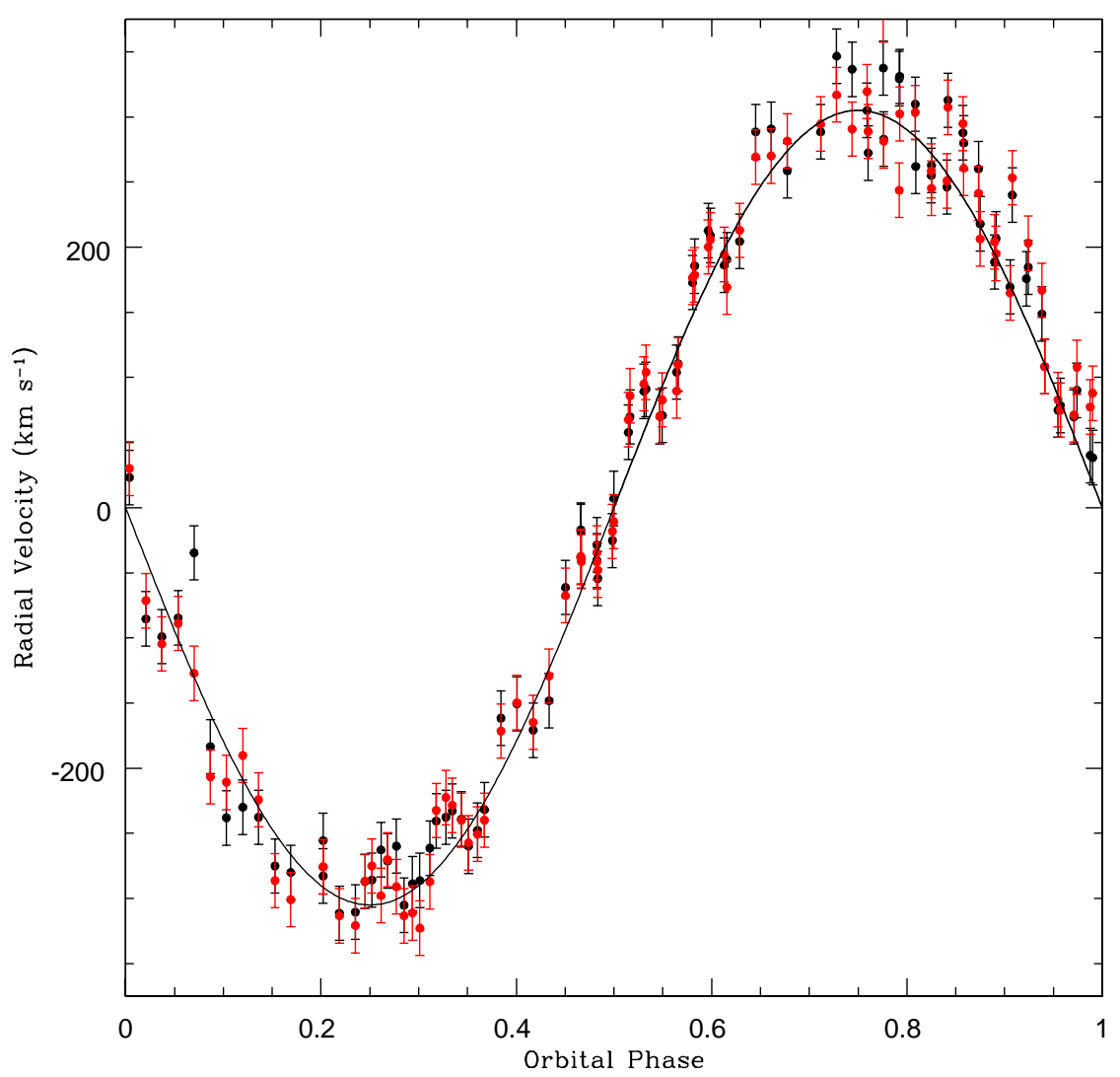

Fig. 15.- The TripleSpec radial velocity curve for U Gem. The black dots are the values for the bluer component of the Na I doublet in the $K$-band, and the red dots are for the red component. A best-fit sinewave with an amplitude of $310 \mathrm{~km} \mathrm{~s}^{-1}$ is superposed. 

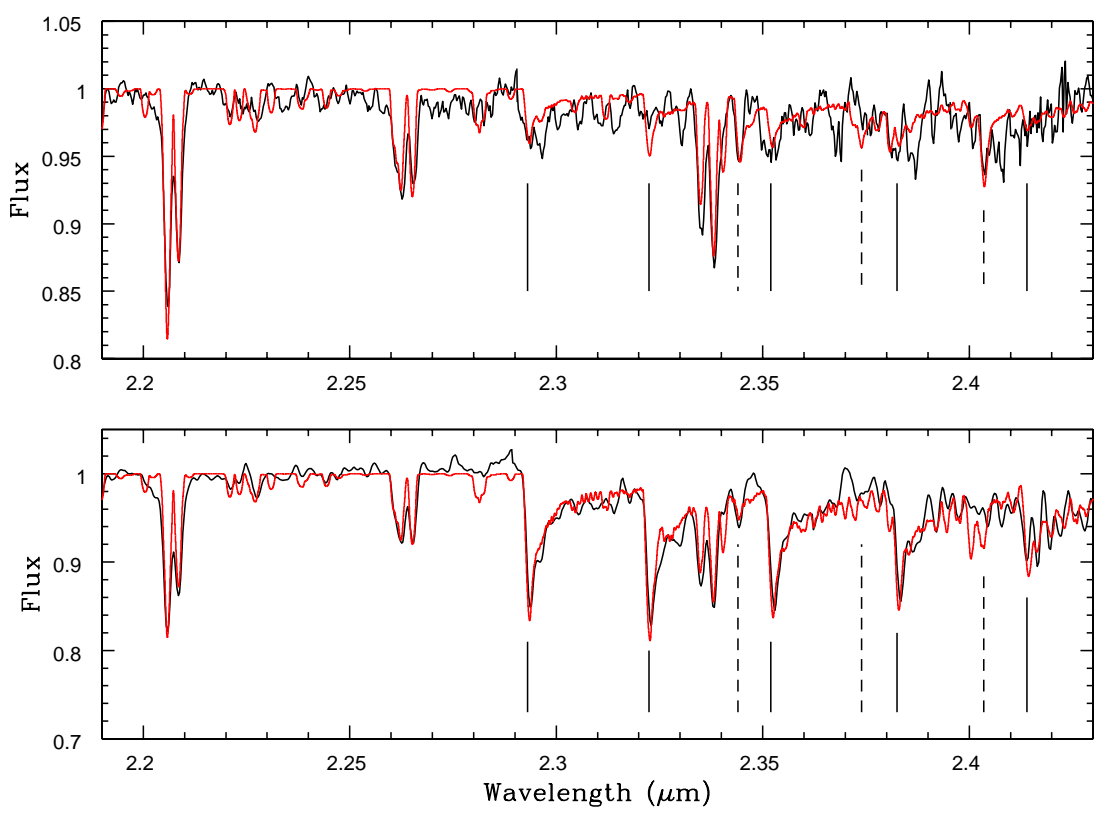

Fig. 16. - The red end of the $K$-band spectrum for U Gem (black, top panel), and GJ 402 (black, bottom panel). We plot a synthetic spectra (red) with $\mathrm{T}_{\text {eff }}=3200 \mathrm{~K}$ and $[\mathrm{Fe} / \mathrm{H}]=$ 0.0 , in black in both panels. The vertical solid lines are the location of the ${ }^{12} \mathrm{CO}$ bandheads, while the vertical dashed lines are for the ${ }^{13} \mathrm{CO}$ features. 

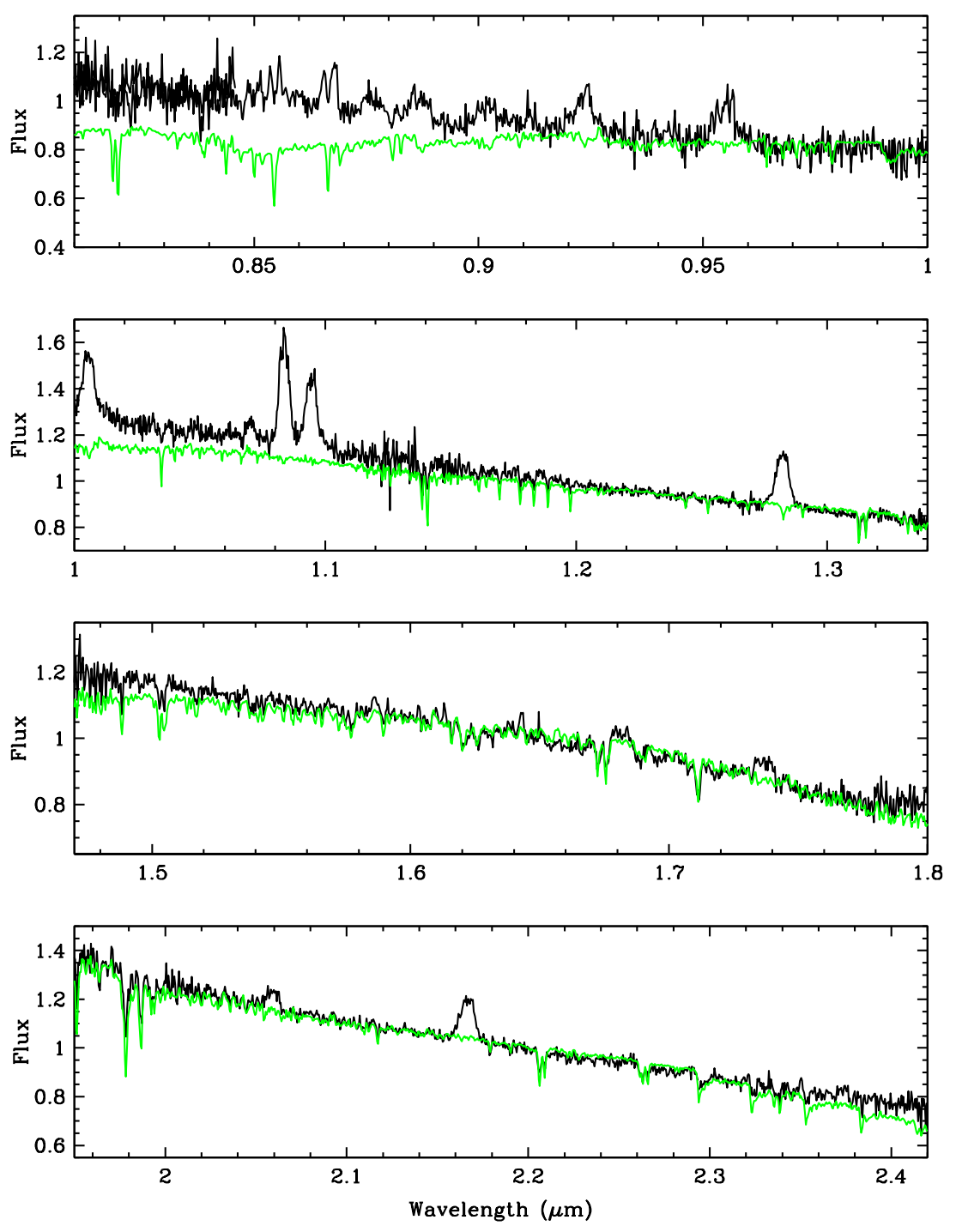

Fig. 17.- The SPEX data for WW Cet (black) compared to the IRTF template Gl 381 $(\mathrm{M} 2.5 \mathrm{~V})$. There is a small amount of contamination from the white dwarf + accretion disk that leads to a blue excess in most of the bands. Note the influence of the Ca II triplet absorption on the H I Paschen continuum. 

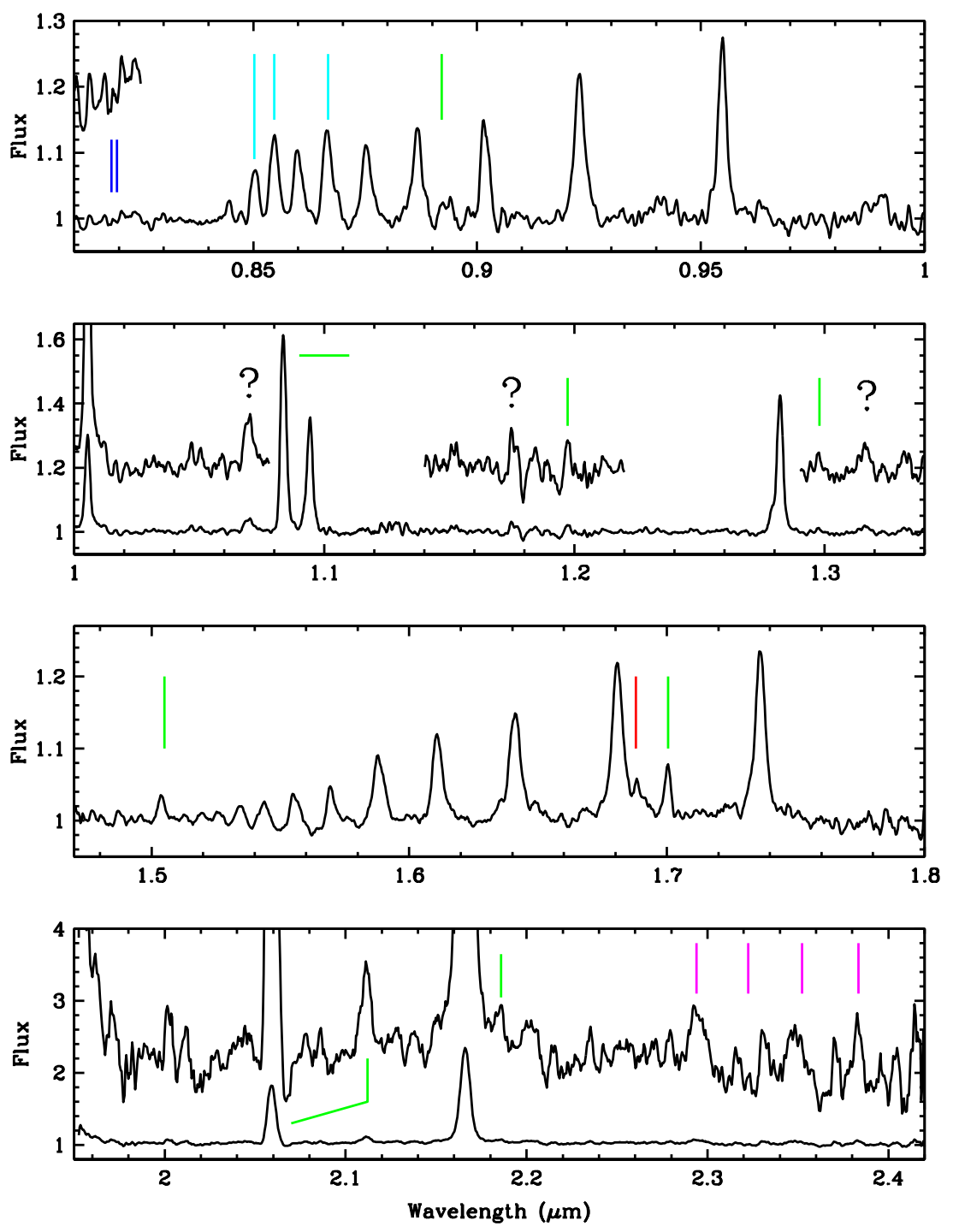

Fig. 18. - The spectrum of LS Peg. The unmarked emission lines are due to H I, while those indicated by green line segments are from helium. The main ${ }^{12} \mathrm{CO}$ bandheads are indicated by vertical magenta lines. [Fe II] is in red, the Ca II triplet in cyan, and the Na I doublet at $0.82 \mu \mathrm{m}$ in blue. Unidentified emission lines are indicated with a "?". 

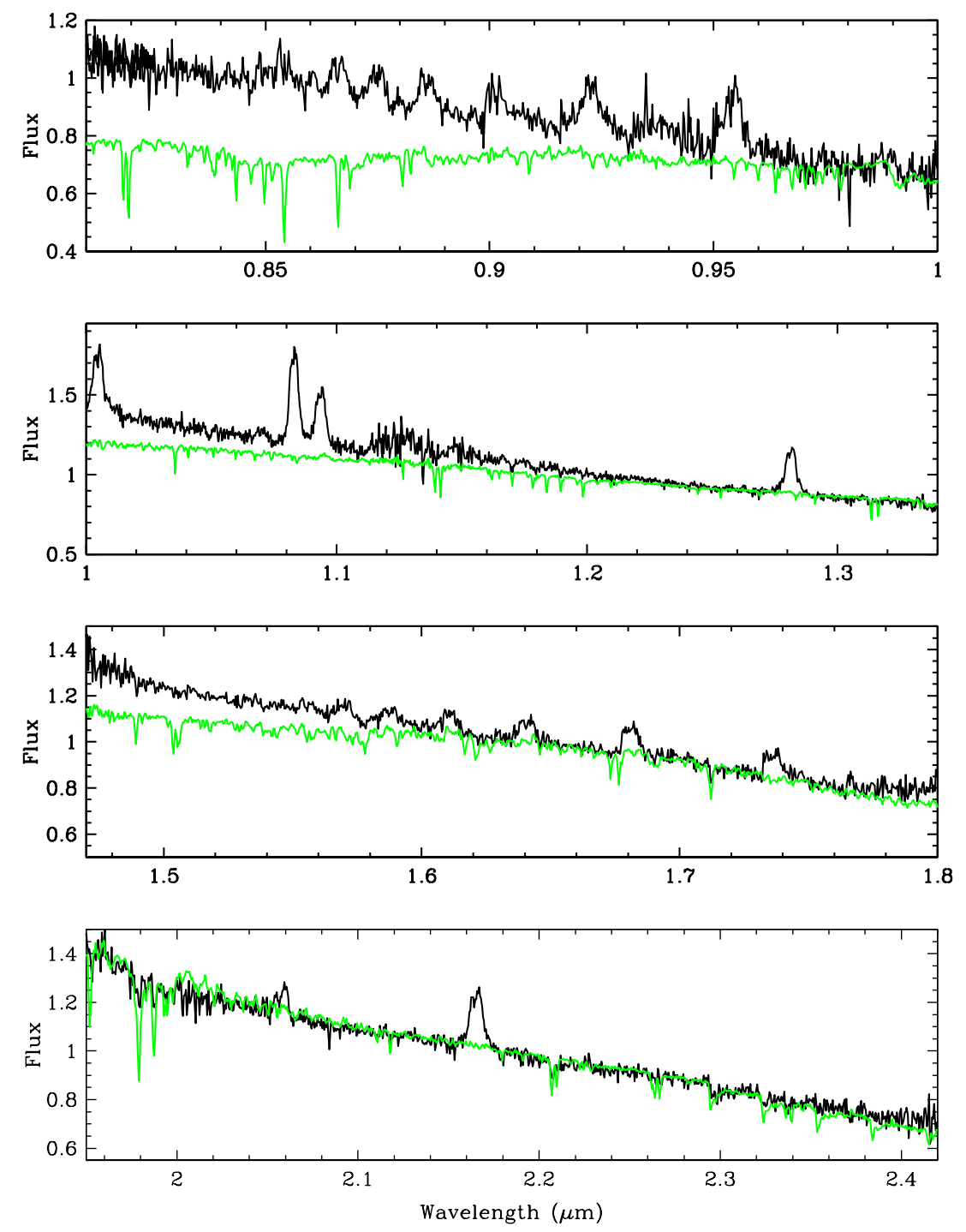

Fig. 19. - The IRTF SPEX data for KT Per (black), and Gl 806 (green). 

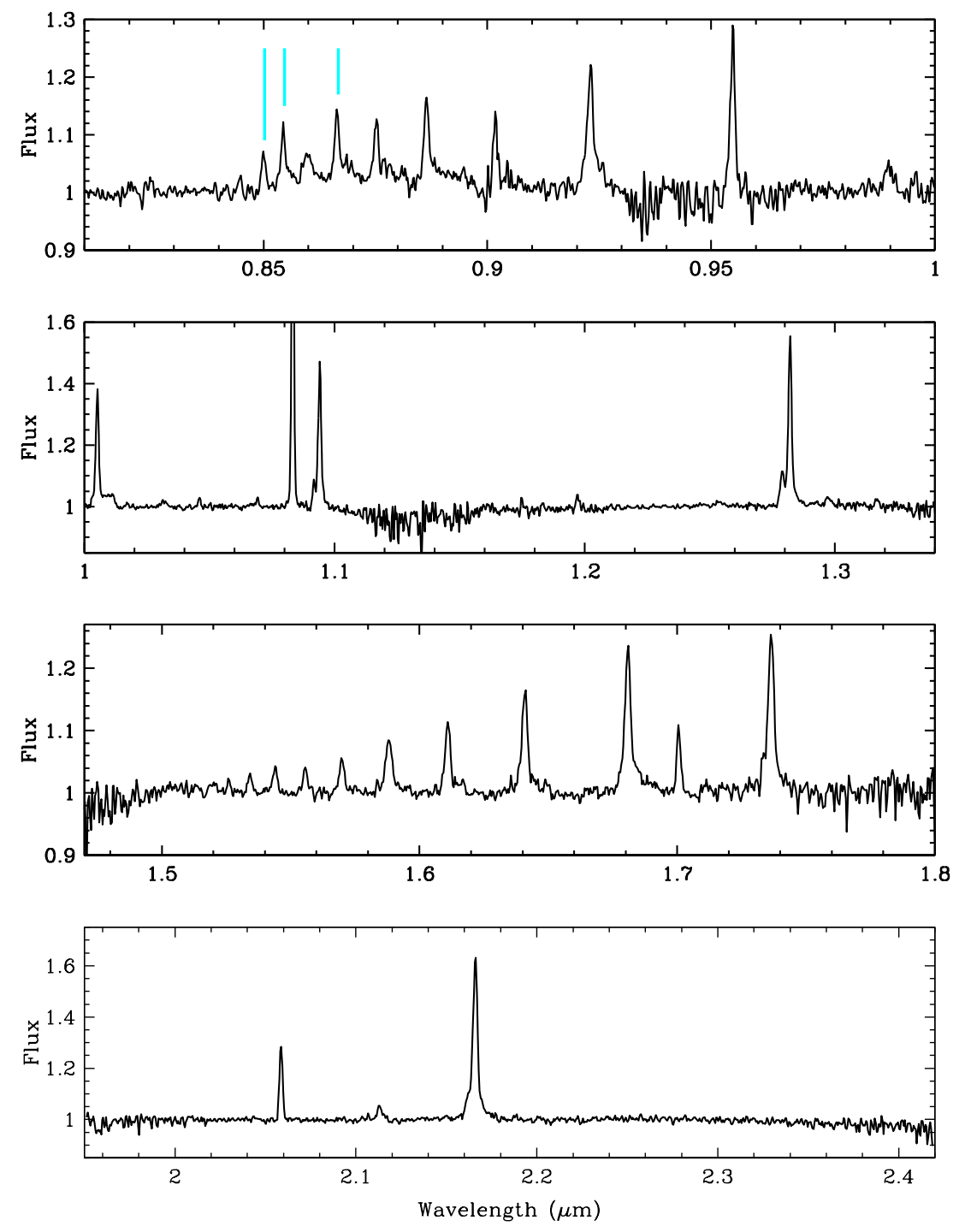

Fig. 20.- The IRTF SPEX data for TT Ari, the location of the Ca II emission lines have been indicated with vertical cyan lines. 


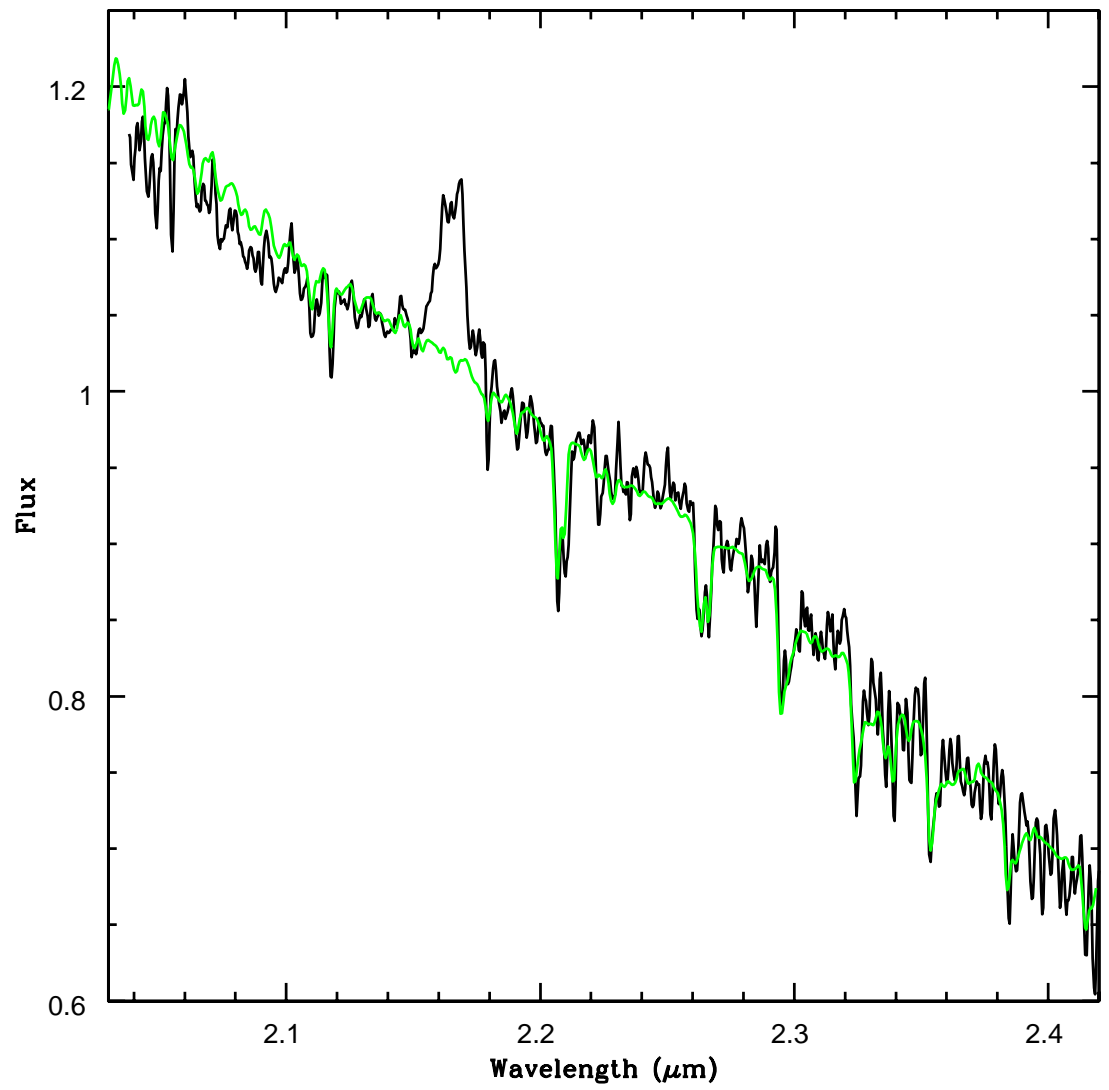

Fig. 21.- The NIRSPEC data for CW Mon (black), compared to the M2V template Gl 806 (green). 


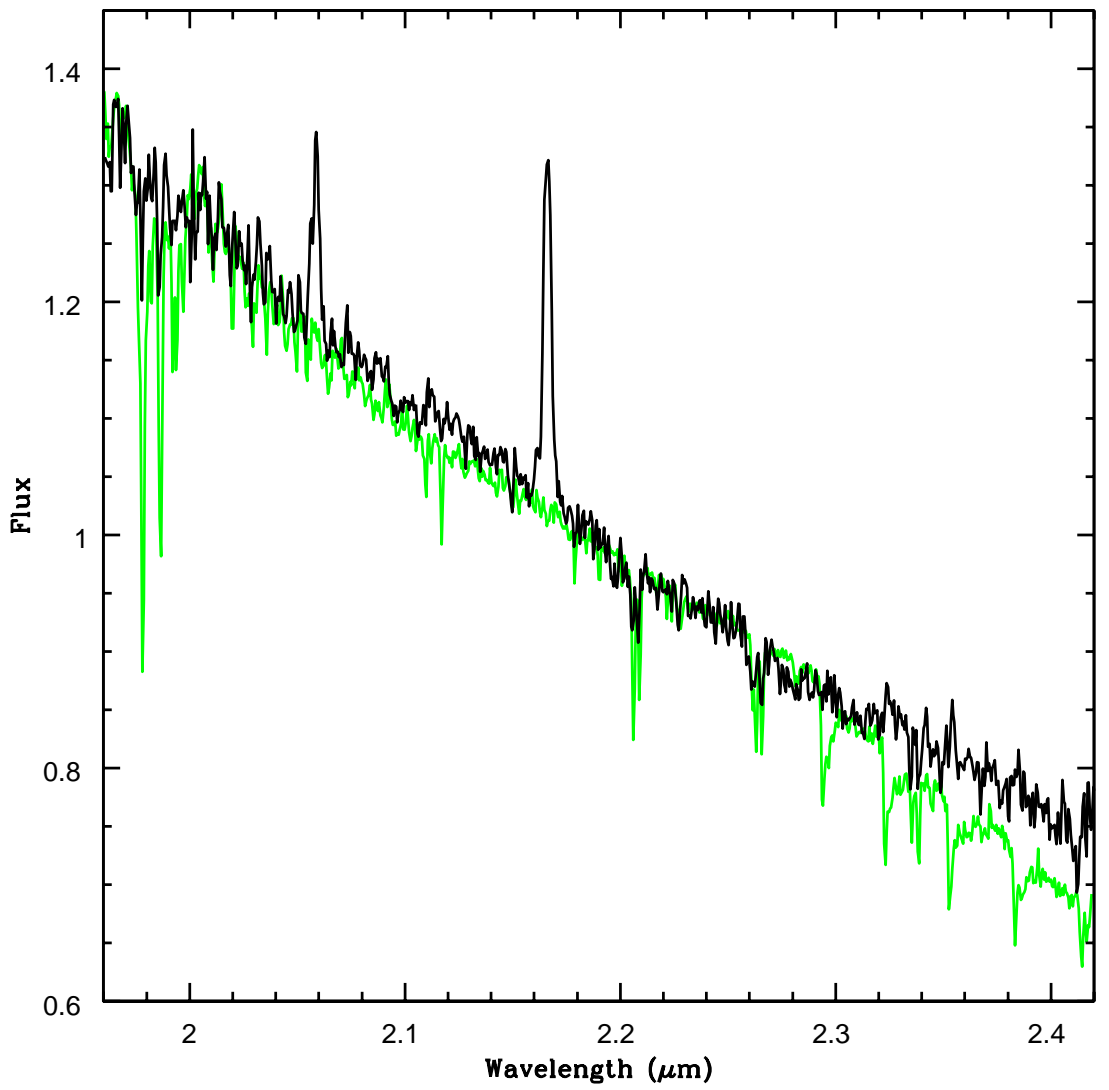

Fig. 22.- The SPEX data for UU Aql (black), compared to the M2V template Gl 806 (green). 


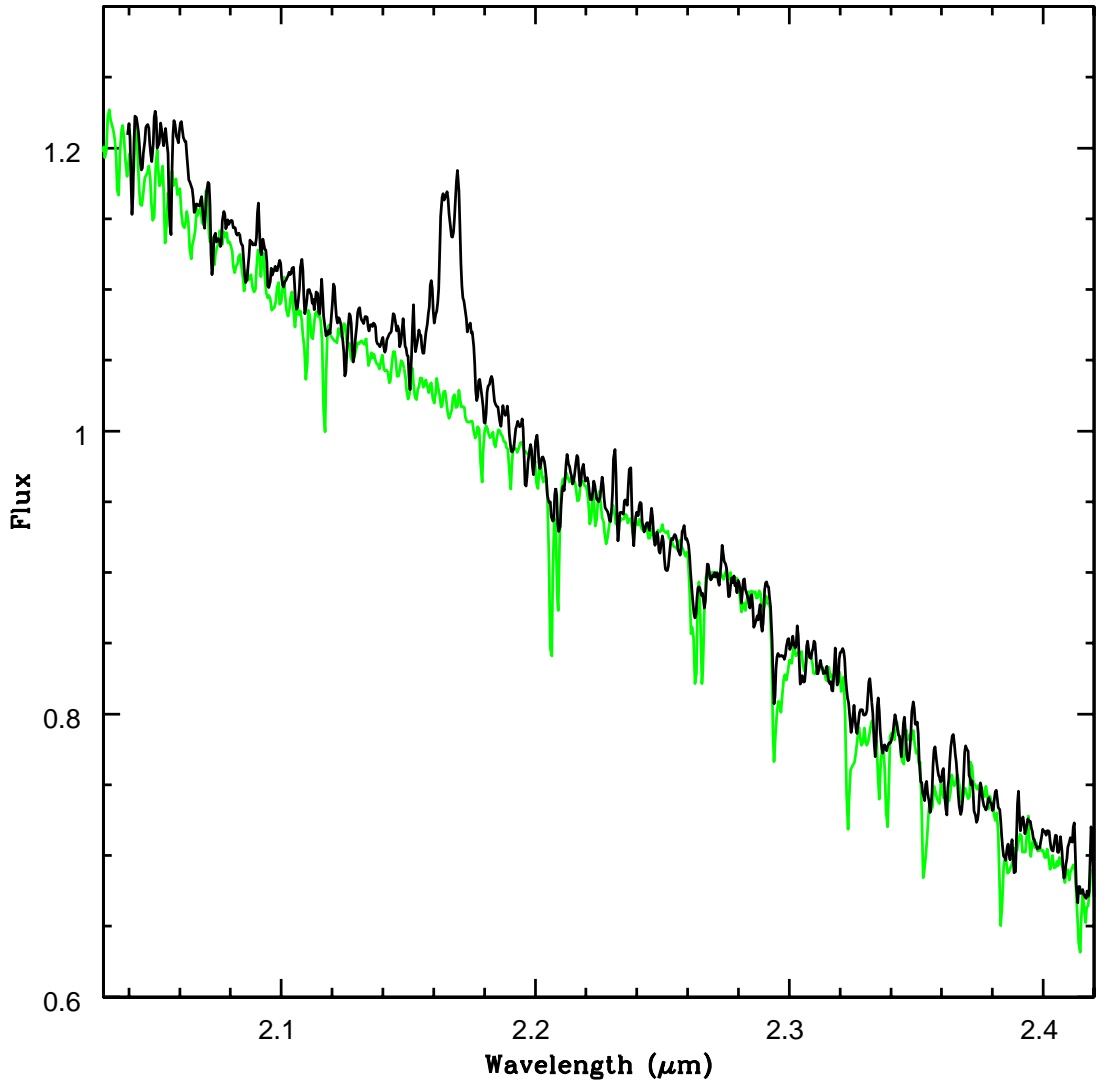

Fig. 23. - The NIRSPEC data for CN Ori (black), compared to the M2V template Gl 806 (green). 


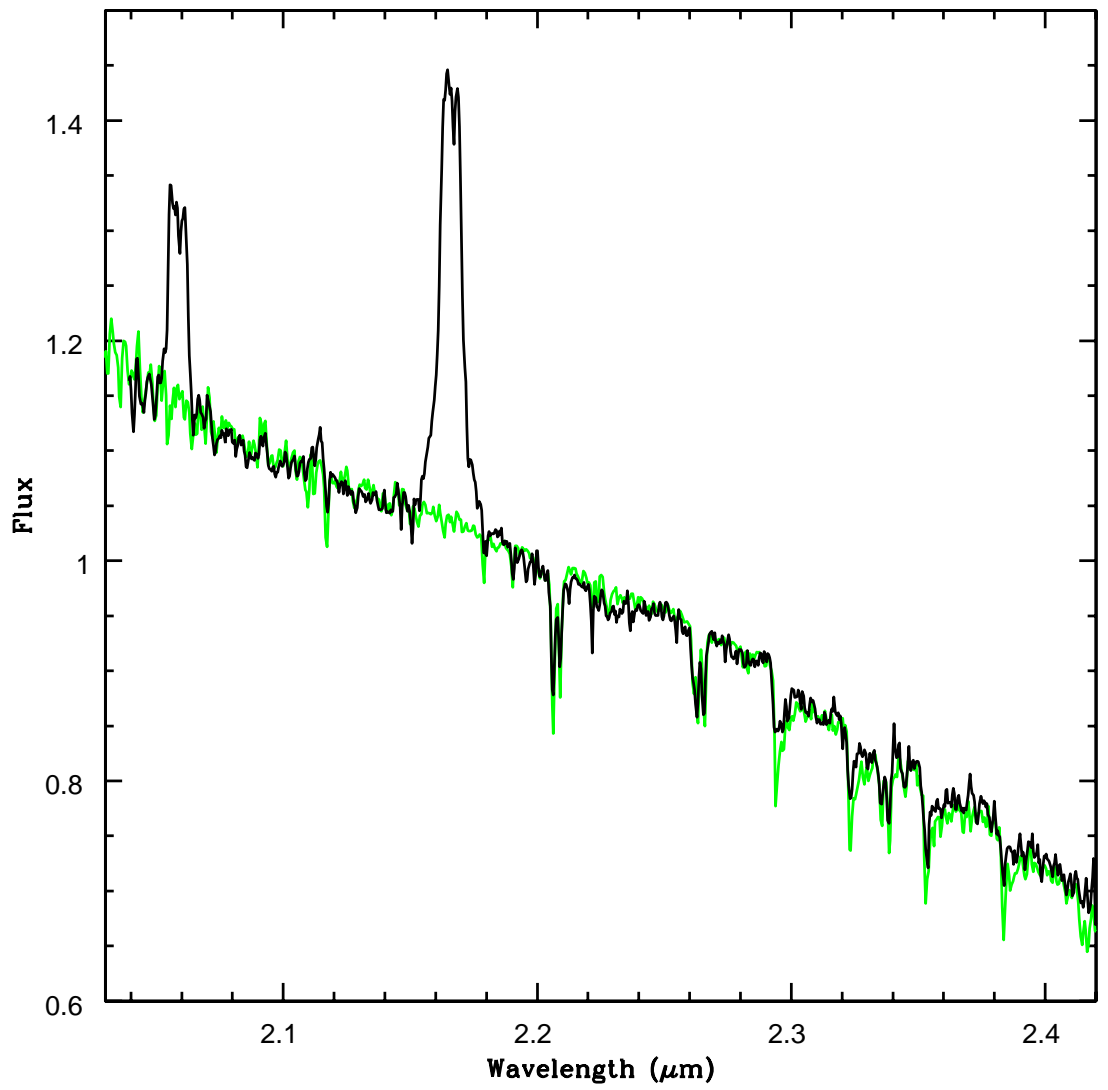

Fig. 24.- The NIRSPEC data for YY/DO Dra (black), compared to the M2.5V template Gl 381 (green). 


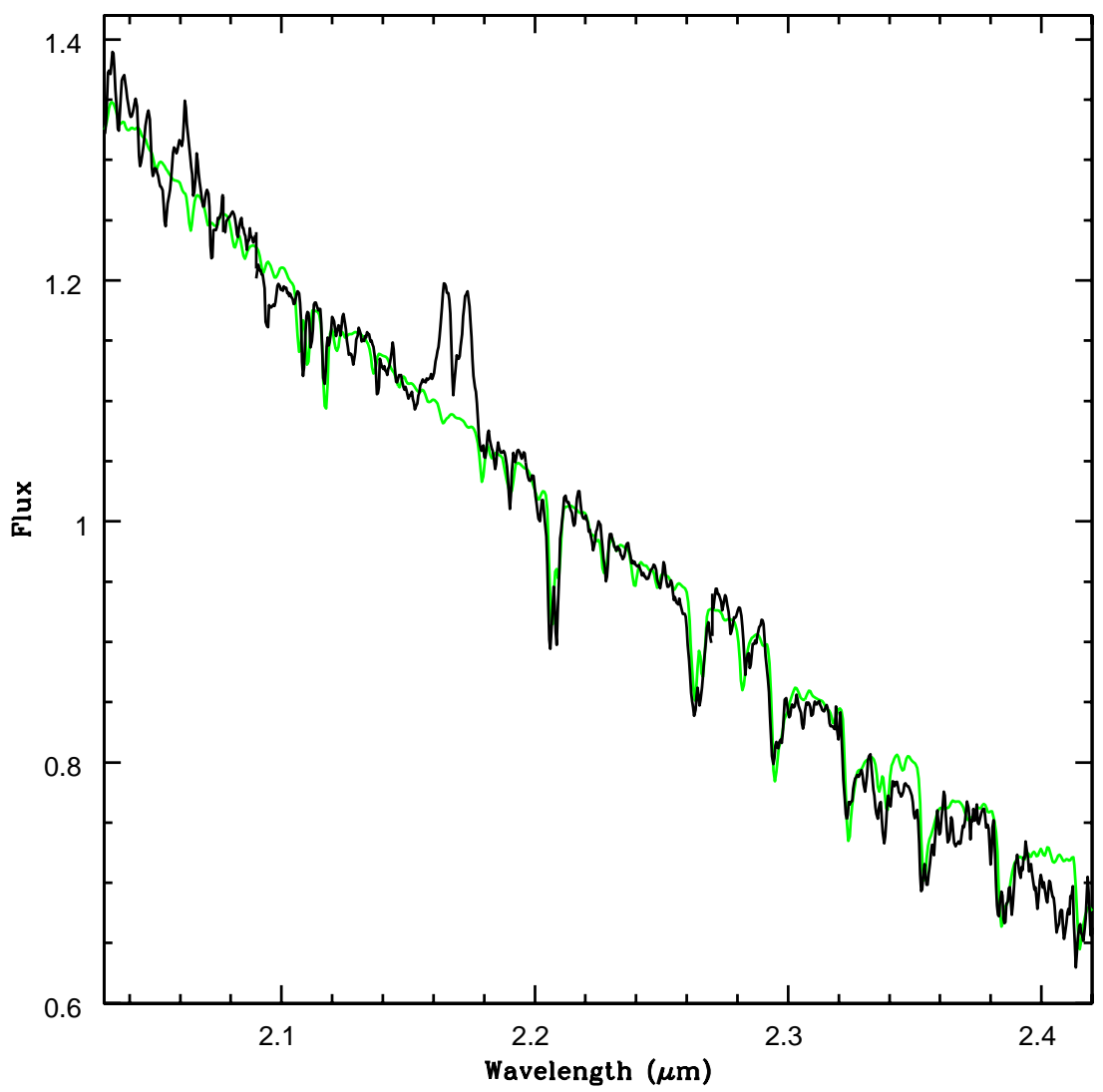

Fig. 25. - The NIRSPEC data for IP Peg (black), compared to the M0V template HD 13905 (green). 


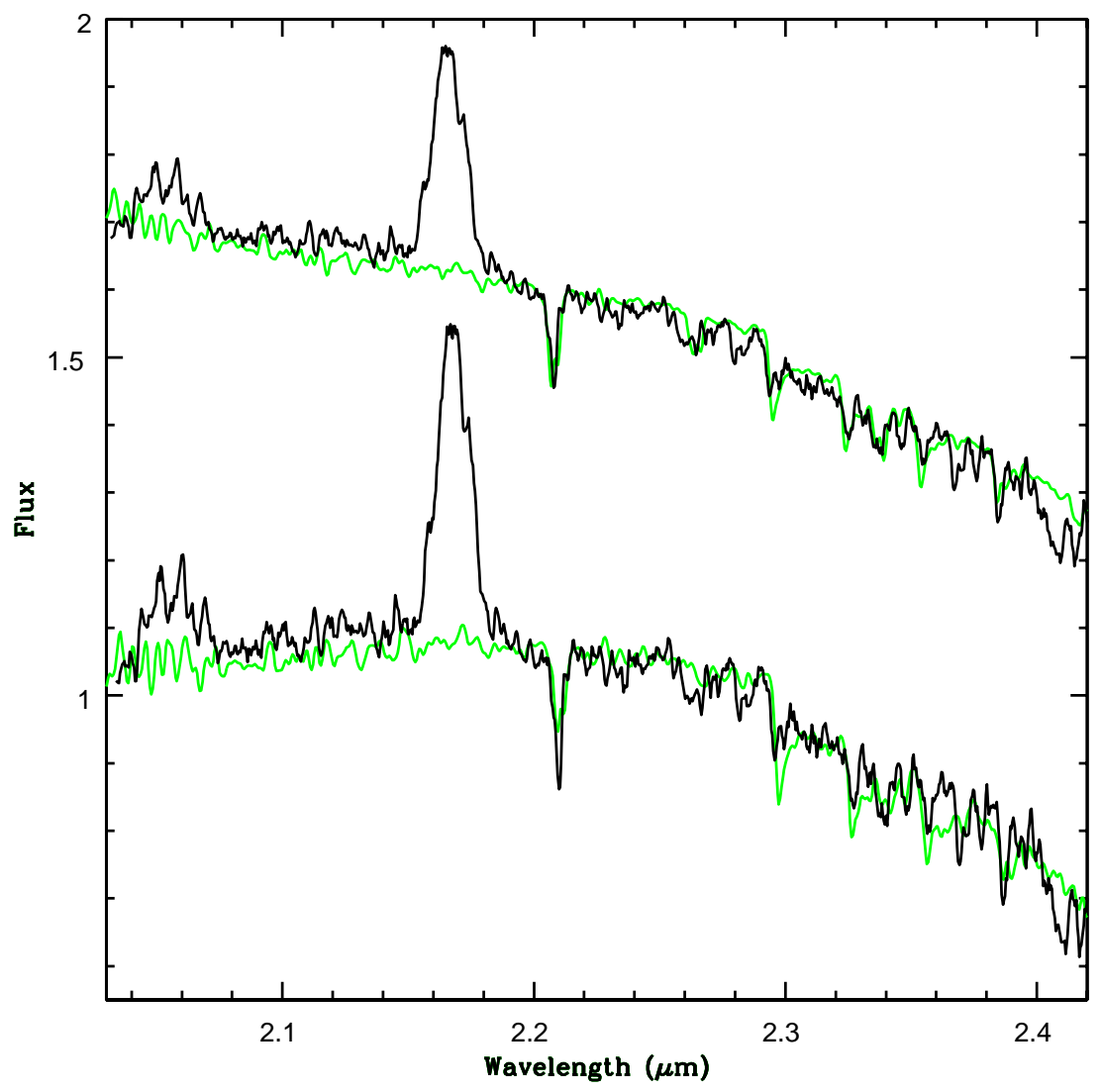

Fig. 26.- The Doppler-corrected NIRSPEC data for RZ Leo (top, black, boxcar smoothed by 5 pixels), compared to (in green) the M4.5V template Gl 268AB. We have subtracted-off a hot blackbody that supplies $30 \%$ of the $K$-band flux from the spectrum of RZ Leo (bottom, black), and compare it to the M6.5V template GJ 1111 (green). 


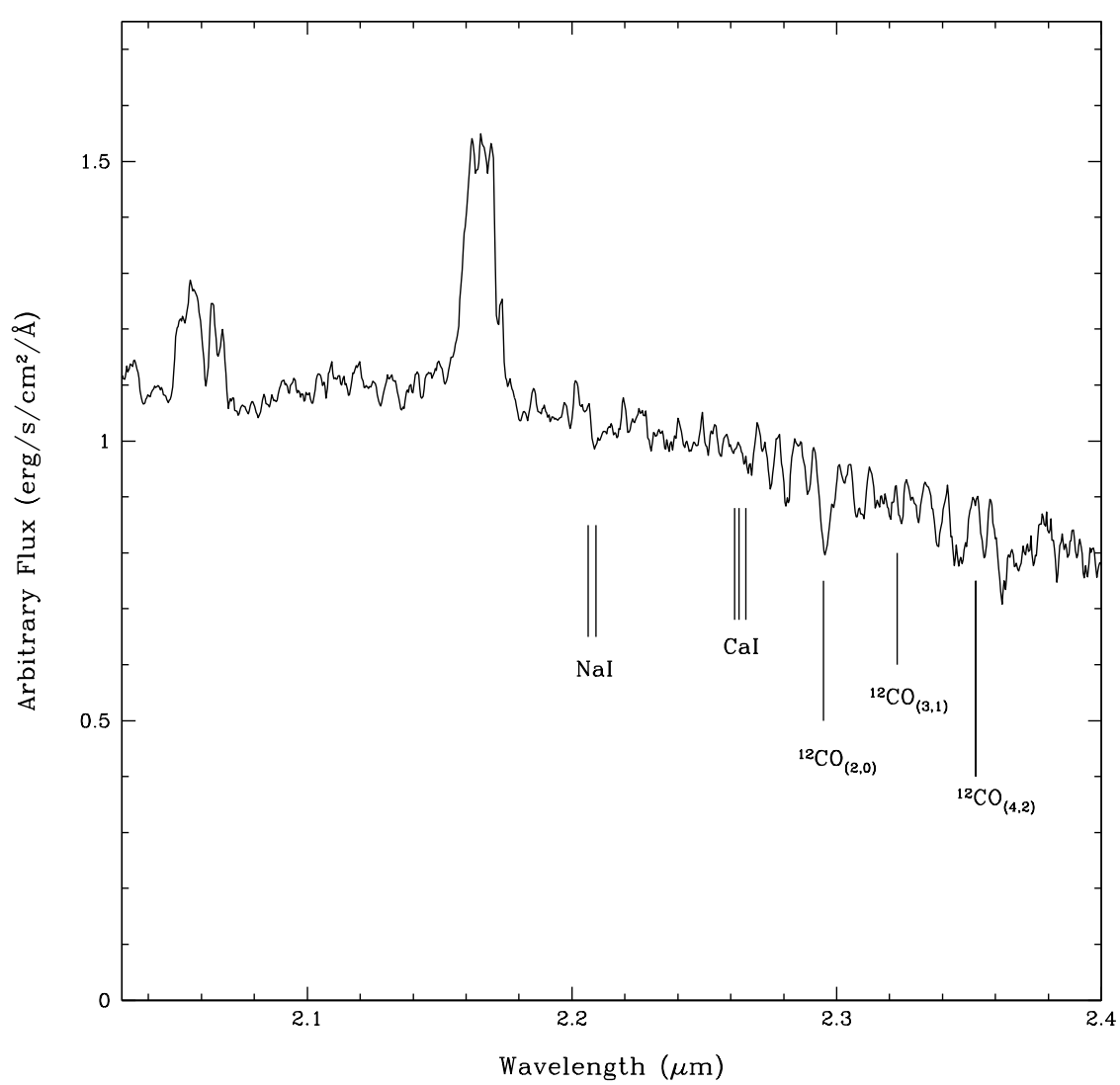

Fig. 27. - The NIRSPEC data for WX Cet (smoothed by $30 \AA$ ). The locations of the most prominent absorption features in late-type dwarfs have been indicated. 

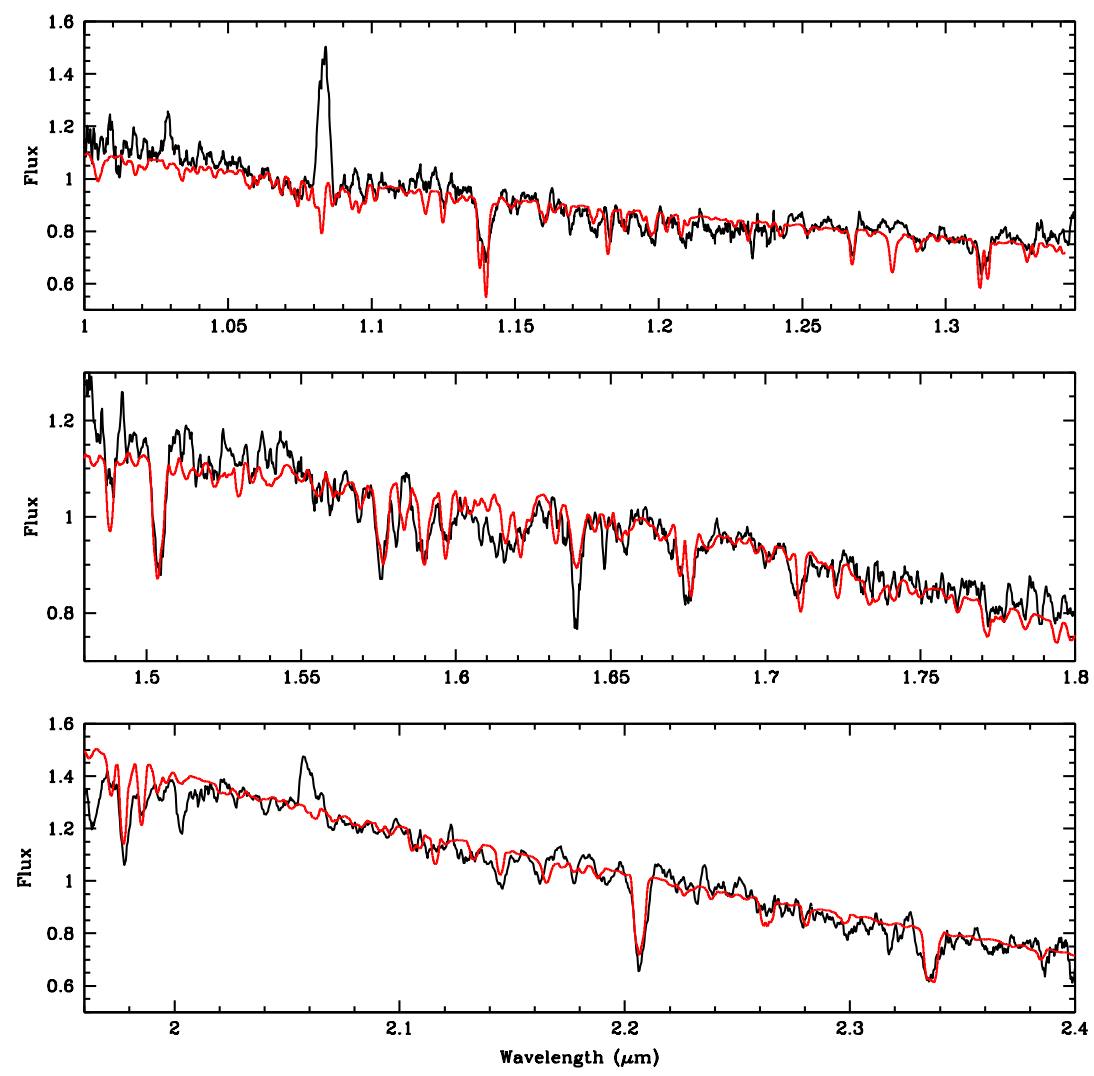

Fig. 28. - The TripleSpec spectrum for QZ Ser (black, smoothed by 20A), and a synthetic spectrum with $\mathrm{T}=4500 \mathrm{~K}$ (red) having the abundance patterns noted in the text. Do to the peculiar nature of the spectrum of QZ Ser, we have not continuum-divided it like the others. Instead, we have multiplied the synthetic spectrum by a $4500 \mathrm{~K}$ blackbody. 


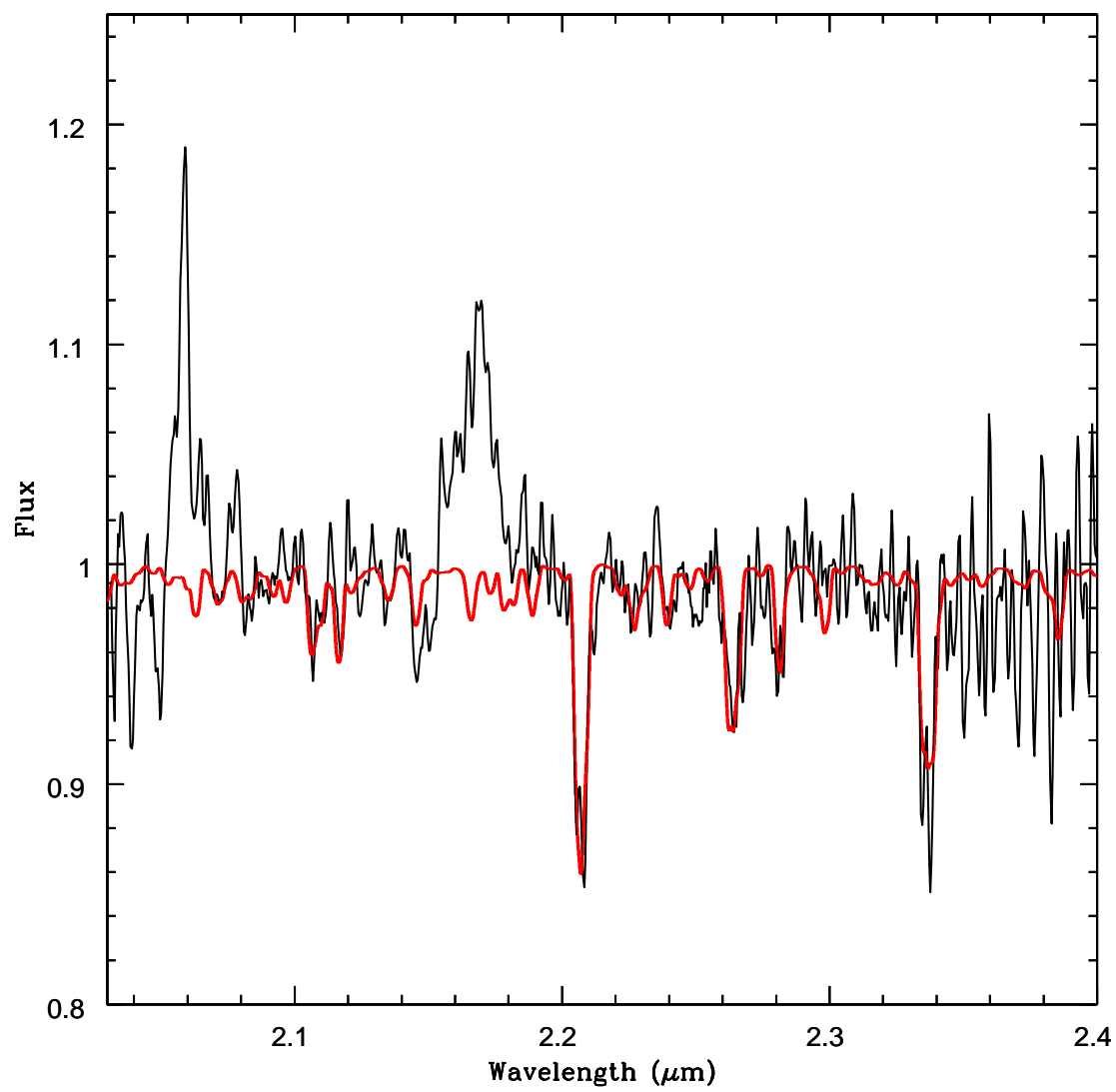

Fig. 29. - The spectrum of EI Psc (black), boxcar smoothed by 3 pixels, and a similarly smoothed synthetic spectrum (red) with $\mathrm{T}=4500 \mathrm{~K}, \log g=4.5$, and $[\mathrm{Fe} / \mathrm{H}]=-0.2$. In this model, sodium is enhanced and carbon is highly deficient. 


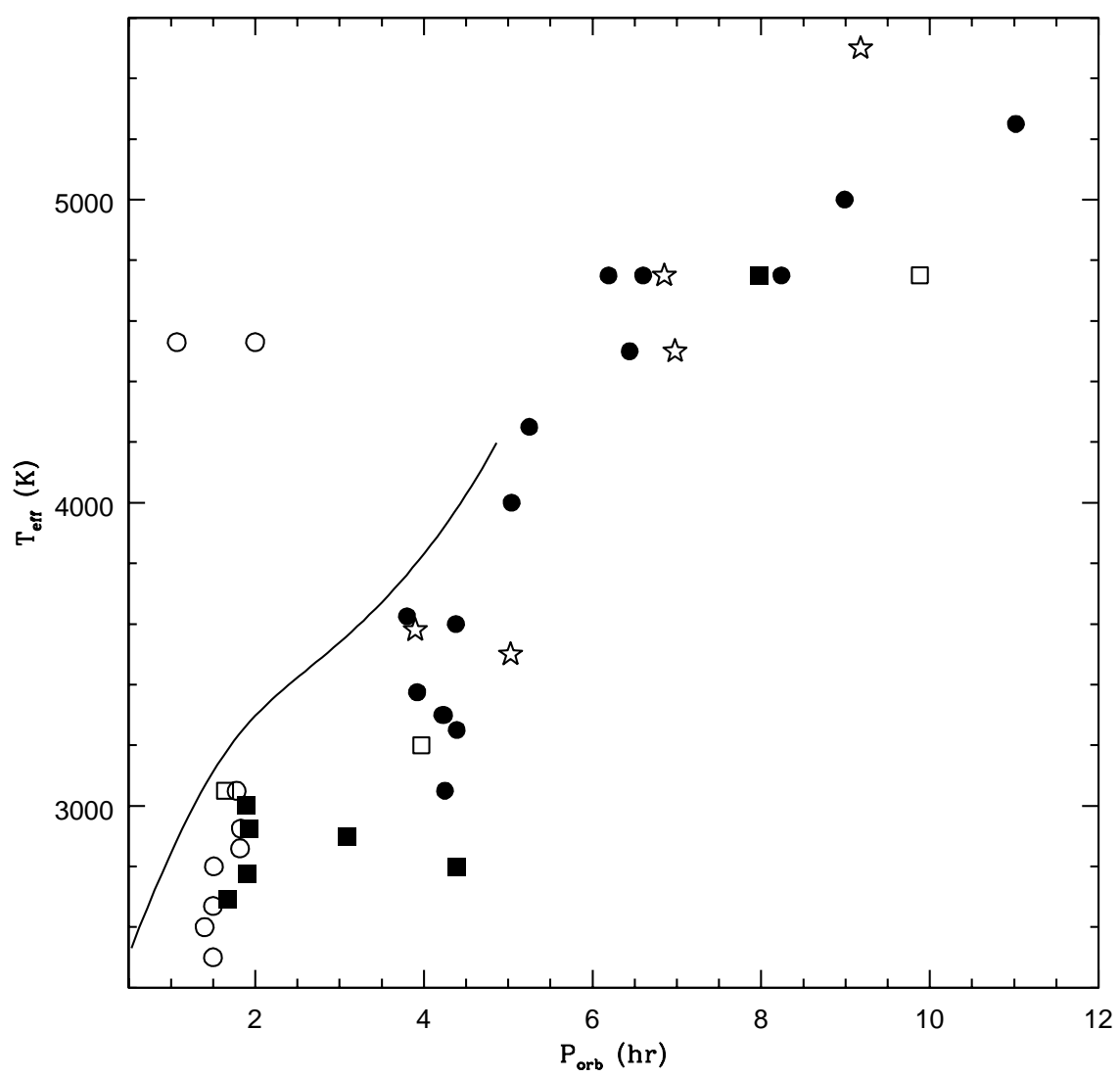

Fig. 30.- The $\mathrm{T}_{\text {eff }}$ vs. $\mathrm{P}_{\text {orb }}$ relationship for $\mathrm{CVs}$ using the results in Table 3 . U Gem-type dwarf novae are plotted as filled circles, while SU UMa type dwarf-novae are plotted as open circles. Z Cam-type dwarf novae are plotted with star symbols. Polars are plotted as filled squares, and IPs as open squares. The solid line was constructed using the mass-radius relationship for late-type main sequence stars from Mann et al. (2013). 